\title{
Primary decomposition in the smooth concordance group of topologically slice knots
}

\author{
Jae Choon Cha \\ Center for Research in Topology, POSTECH, Pohang 37673, Republic of Korea, and School of Mathematics, Korea Institute for \\ Advanced Study, Seoul 02455, Republic of Korea, E-mail: jccha@ postech.ac.kr.
}

Received: 12 February 2020; Revised: 28 April 2021; Accepted: 27 June 2021

2020 Mathematics Subject Classification: Primary - 57M25; Secondary - 57M27, 57N70

\begin{abstract}
We address primary decomposition conjectures for knot concordance groups, which predict direct sum decompositions into primary parts. We show that the smooth concordance group of topologically slice knots has a large subgroup for which the conjectures are true and there are infinitely many primary parts, each of which has infinite rank. This supports the conjectures for topologically slice knots. We also prove analogues for the associated graded groups of the bipolar filtration of topologically slice knots. Among ingredients of the proof, we use amenable $L^{2}$ signatures, Ozsváth-Szabó $d$-invariants and Némethi's result on Heegaard Floer homology of Seifert 3-manifolds. In an appendix, we present a general formulation of the notion of primary decomposition.
\end{abstract}

\section{Introduction and main results}

It is a major open problem to classify knots in 3-space modulo concordance. Our understanding is far from complete, for both topological and smooth knot concordance groups. The sophistication of the smooth case beyond topological concordance is measured by the smooth concordance group of topologically slice knots, which has been actively investigated using modern smooth techniques.

In the study of knot concordance, the notion of primary decomposition first appeared in Jerome Levine's foundational work [35, 34]. Briefly, he constructed an algebraic concordance group of Seifert matrices and proved that it is isomorphic to the knot concordance group in high odd dimensions, whereas it gives algebraic invariants in the classical dimension [35]. He proved that a rational coefficient version of the algebraic concordance group decomposes into a direct sum of certain 'primary parts' indexed by irreducible factors of Alexander polynomials. This plays a crucial role in his well-known classification result that the algebraic concordance group and high odd-dimensional knot concordance groups are isomorphic to $\mathbb{Z}^{\infty} \oplus\left(\mathbb{Z}_{2}\right)^{\infty} \oplus\left(\mathbb{Z}_{4}\right)^{\infty}[34]$.

For the low-dimensional case, it is natural to ask whether the classical knot concordance groups and related objects admit analogous primary decomposition and to study the structures via primary parts. In an appendix, we formulate a general notion of primary decomposition, which specialises to several specific cases including concordance and rational homology cobordism, and discuss related questions. We hope this is useful for future study as well. The appendix also discusses known earlier results from the viewpoint of the general formulation. In particular, for topological knot concordance, there were remarkable results related to primary decomposition [36, 28, 29, 14, 13, 30].

In this article, we begin a detailed study of the smooth concordance group of topologically slice knots $\mathcal{T}$, via primary decomposition. Precise statements of our results are given in Subsections 1.1 and 1.2.

(C) The Author(s), 2021. Published by Cambridge University Press. This is an Open Access article, distributed under the terms of the Creative Commons Attribution licence (http://creativecommons.org/licenses/by/4.0/), which permits unrestricted re-use, distribution, and reproduction in any medium, provided the original work is properly cited. 
Briefly, the conjectural primary decompositions (see Question 1.1) are direct sum decompositions, along irreducible factors of Alexander polynomials, of the quotient $\mathcal{T} / \Delta$ where $\Delta$ is the subgroup generated by knots with unit Alexander polynomials. (Taking the quotient by $\Delta$ is along the same lines of ignoring units for factorisations in a ring.) We show that the conjectures hold for a large subgroup of $\mathcal{T}$ and that there are infinitely many primary parts, each of which has infinite rank (see Theorems A and B). This provides evidence supporting primary decomposition conjectures and reveals a rich structure in $\mathcal{T} / \Delta$, generalising a result of Hedden, Livingston and Ruberman [25] that $\mathcal{T} / \Delta$ has infinite rank.

In proving this, the essential challenge is to find irreducible polynomials $\lambda(t)$ and topologically slice knots (whose nontrivial linear combinations are) not concordant to any knot whose Alexander polynomial is relatively prime to $\lambda(t)$. It appears to be hard, if possible at all, to do this using known smooth invariants such as those from gauge theory, Heegaard Floer homology and Khovanov homology. Our proof combines amenable $L^{2}$-signatures, which are key ingredients of recent studies of topological concordance, with smooth information from Heegaard Floer homology of infinitely many branched covers of a knot. It seems intriguing to study whether more recent smooth invariants are useful in understanding primary decomposition, motivated from the results and approaches of this article.

We also prove results that support primary decomposition conjectures for the bipolar filtration of topologically slice knots (see Theorems C and D).

\subsection{Primary decomposition for topologically slice knots}

In what follows, we state primary decomposition conjectures and main results for $\mathcal{T}$.

For a knot $K$ in $S^{3}$, denote the Alexander polynomial by $\Delta_{K}$ and regard it as an element in the Laurent polynomial ring $\mathbb{Q}\left[t^{ \pm 1}\right]$. Then $\Delta_{K}$ is well defined up to associates. Recall that $\lambda$ and $\mu \in \mathbb{Q}\left[t^{ \pm 1}\right]$ are associates if $\lambda=a t^{k} \mu$ for some $a \in \mathbb{Q} \backslash\{0\}$ and $k \in \mathbb{Z}$. The standard involution on $\mathbb{Q}\left[t^{ \pm 1}\right]$ is defined by $\left(\sum a_{i} t^{i}\right)^{*}=\sum a_{i} t^{-i}$. We say that $\lambda$ and $\mu \in \mathbb{Q}\left[t^{ \pm 1}\right]$ are $*$-associates if $\lambda$ is an associate of either $\mu$ or $\mu^{*}$.

Denote the smooth concordance class of a knot $K$ by $[K]$. Recall that $\Delta=\left\{[K] \in \mathcal{T} \mid \Delta_{K}\right.$ is trivial $\}$. For an irreducible $\lambda$ in $\mathbb{Q}\left[t^{ \pm 1}\right]$, let

$$
\begin{aligned}
\mathcal{T}_{\lambda} & =\left\{[K] \in \mathcal{T} \mid \Delta_{K} \text { is an associate of }\left(\lambda \lambda^{*}\right)^{k} \text { for some } k \geq 0\right\}, \\
\mathcal{T}^{\lambda} & =\left\{[K] \in \mathcal{T} \mid \Delta_{K} \text { is relatively prime to } \lambda\right\} .
\end{aligned}
$$

We remark that the product $\lambda \lambda^{*}$ in the definition of $\mathcal{T}_{\lambda}$ reflects the Fox-Milnor condition that $\Delta_{K}$ is an associate of $f f^{*}$ for some $f \in \mathbb{Q}\left[t^{ \pm 1}\right]$ when $K$ is topologically slice. Note that $\mathcal{T}_{\lambda}$ and $\mathcal{T}^{\lambda}$ are subgroups containing $\Delta$ for every irreducible $\lambda$. Also, $\mathcal{T}_{\lambda}=\mathcal{T}_{\mu}$ and $\mathcal{T}^{\lambda}=\mathcal{T}^{\mu}$ if $\lambda$ and $\mu$ are $*$-associates.

Primary decomposition for topologically slice knots concerns natural homomorphisms

$$
\Phi_{L}: \bigoplus_{[\lambda]} \mathcal{T}_{\lambda} / \Delta \longrightarrow \mathcal{T} / \Delta \quad \text { and } \quad \Phi_{R}: \mathcal{T} / \Delta \longrightarrow \bigoplus_{[\lambda]} \mathcal{T} / \mathcal{T}^{\lambda}
$$

Here the index $[\lambda]$ of the direct sums varies over the $*$-associate classes of irreducibles $\lambda$ that arise as the factor of an Alexander polynomial of a knot. The homomorphism $\Phi_{L}$ is defined to be the sum of the inclusions $\mathcal{T}_{\lambda} / \Delta \hookrightarrow \mathcal{T} / \Delta$. Because $\Delta_{K}$ is a product of finitely many irreducibles, the quotient epimorphisms $\mathcal{T} / \Delta \rightarrow \mathcal{T} / \mathcal{T}^{\lambda}$ induce a homomorphism into the direct sum, which is our $\Phi_{R}$ above. This formulation is influenced by earlier work in the literature, particularly Levine [35, 34] and Cochran, Harvey and Leidy [14, 13].

An informal remark. One might regard elements in $\mathcal{T}_{\lambda} / \Delta \subset \mathcal{T} / \Delta$ as ' $\lambda$-primary' and $\mathcal{T} / \Delta \rightarrow \mathcal{T} / \mathcal{T}^{\lambda}$ as 'forgetting those coprime to $\lambda$ ' or 'extracting the $\lambda$-primary component' of an element. Then, the surjectivity of $\Phi_{L}$ means the existence of a 'decomposition into a sum of primary elements', and the injectivity of $\Phi_{L}$ means the uniqueness of such a decomposition. Also, the injectivity of $\Phi_{R}$ means that 'primary components determine an element uniquely', and the surjectivity of $\Phi_{R}$ means that 'every combination of primary components is realisable'. 
Question 1.1 (Primary decomposition for topologically slice knots).

(1) Left primary decomposability: is $\Phi_{L}$ an isomorphism?

(2) Right primary decomposability: is $\Phi_{R}$ an isomorphism?

More generally (if they are not isomorphisms), what are their kernel and cokernel?

We conjecture an affirmative answer to (2) and that $\Phi_{L}$ is injective at the least. In any case, it appears to be interesting to study $\mathcal{T}_{\lambda} / \Delta$ and $\mathcal{T} / \mathcal{T}^{\lambda}$, which we call left and right primary parts (or primary factors). This leads us particularly to the following.

(3) Nontriviality of primary parts: are $\mathcal{T}_{\lambda} / \Delta$ and $\mathcal{T} / \mathcal{T}^{\lambda}$ nonzero for each irreducible $\lambda$ that arises as the factor of an Alexander polynomial of a knot?

More generally (if they are nontrivial), what are the isomorphism types of the primary parts $\mathcal{T}_{\lambda} / \Delta$ and $\mathcal{T} / \mathcal{T}^{\lambda}$ ?

(4) Relationship of left and right primary parts: is the composition

$$
\mathcal{T}_{\lambda} / \Delta \hookrightarrow \mathcal{T} / \Delta \rightarrow \mathcal{T} / \mathcal{T}^{\lambda}
$$

an isomorphism?

We remark that Definition A.1 in the appendix generalises the notion of left and right primary decomposition to a broader context. Also, regarding the relationship of Question 1.1(1) and (2), see Lemma A.4 in the appendix.

The first main result of this article, which is given as Theorem A, says that there is a large subgroup of $\mathcal{T}$ for which the answers to the above questions are affirmative and many primary parts of $\mathcal{T}$ are highly nontrivial. To state the result, we use the following notation. For a subgroup $\mathcal{S}$ of $\mathcal{T}$ and an irreducible $\lambda \in \mathbb{Q}\left[t^{ \pm 1}\right]$, let $\mathcal{S}_{\lambda}$ be the subgroup of $[K] \in \mathcal{S}$ with $\Delta_{K}$ a power of $\lambda \lambda^{*}$ and $\mathcal{S}^{\lambda}$ be the subgroup of $[K] \in \mathcal{S}$ with $\operatorname{gcd}\left(\Delta_{K}, \lambda\right)=1$. That is, $\mathcal{S}_{\lambda}=\mathcal{S} \cap \mathcal{T}_{\lambda}$ and $\mathcal{S}^{\lambda}=\mathcal{S} \cap \mathcal{T}^{\lambda}$. Then one can ask Question 1.1 for $\mathcal{S}$ in place of $\mathcal{T}$. Let

$$
\Lambda=\{(m+1) t-m \mid m \text { is a positive integer }\} .
$$

Note that $\Lambda$ is an infinite collection of pairwise non-*-associate irreducibles $\lambda$ such that $\lambda \lambda^{*}$ is an Alexander polynomial of a knot.

Theorem A. There is a subgroup $\mathcal{S}$ in $\mathcal{T}$ containing $\Delta$ that satisfies the following:

(1) For every $\lambda \in \Lambda, \mathcal{S}_{\lambda} / \Delta \cong \mathbb{Z}^{\infty}, \mathcal{S} / \mathcal{S}^{\lambda} \cong \mathbb{Z}^{\infty}$ and the composition $\mathcal{S}_{\lambda} / \Delta \hookrightarrow \mathcal{S} / \Delta \rightarrow \mathcal{S} / \mathcal{S}^{\lambda}$ is an isomorphism.

(2) The inclusions $\mathcal{S}_{\lambda} / \Delta \rightarrow \mathcal{S} / \Delta$ induce an isomorphism $\bigoplus_{\lambda \in \Lambda} \mathcal{S}_{\lambda} / \Delta \rightarrow \mathcal{S} / \Delta$.

(3) The surjections $\mathcal{S} / \Delta \rightarrow \mathcal{S} / \mathcal{S}^{\lambda}$ induce an isomorphism $\mathcal{S} / \Delta \rightarrow \bigoplus_{\lambda \in \Lambda} \mathcal{S} / \mathcal{S}^{\lambda}$.

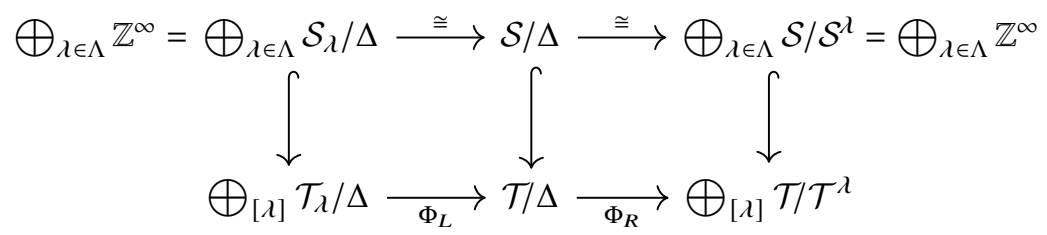

From Theorem $A$ it follows that each of the primary parts $\mathcal{T}_{\lambda} / \Delta$ and $\mathcal{T} / \mathcal{T}^{\lambda}$ has a subgroup isomorphic to $\mathbb{Z}^{\infty}$ for all $\lambda \in \Lambda$. An immediate consequence is the main result of [25] that $\mathcal{T} / \Delta$ has a subgroup isomorphic to $\mathbb{Z}^{\infty}$. 
Note that Theorem A(1) implies the following.

Theorem B. For each $\lambda \in \Lambda$, there is an infinite collection of topologically slice knots $\left\{K_{\lambda, i}\right\}_{i=1}^{\infty}$ with $\Delta_{K_{\lambda, i}}$ a power of $\lambda \lambda^{*}$, such that any nontrivial linear combination of the $K_{\lambda, i}$ is not smoothly concordant to any knot $J$ with $\Delta_{J}$ relatively prime to $\lambda$.

Indeed, Theorem B is equivalent to Theorem A, by an elementary formal argument.

Proof that Theorem B implies Theorem A. Suppose that Theorem B holds. Let $\mathcal{S}$ be the subgroup in $\mathcal{T}$ that is generated by $\Delta$ and the two-parameter family $\left\{K_{\lambda, i}\right\}_{\lambda \in \Lambda, i \in \mathbb{N}}$ given by Theorem B.

We claim that, for each $\lambda \in \Lambda, \mathcal{S}_{\lambda}$ is equal to the subgroup generated by $\Delta$ and the one-parameter family $\left\{K_{\lambda, i}\right\}_{i \in \mathbb{N}}$. To see this, first observe that for irreducibles $\lambda$ and $\mu$ that are not $*$-associates, $\mathcal{S}_{\lambda} \subset \mathcal{S}^{\mu}$, and so the composition

$$
\mathcal{S}_{\lambda} / \Delta \hookrightarrow \mathcal{S} / \Delta \rightarrow \mathcal{S} / \mathcal{S}^{\mu}
$$

is zero. If a linear combination $[J]+\sum_{\mu} \sum_{i} r_{\mu, i}\left[K_{\mu, i}\right]\left([J] \in \Delta, r_{\mu, i} \in \mathbb{Z}\right)$ lies in $\mathcal{S}_{\lambda}$, then for each $\mu \neq \lambda$, the image of the linear combination in $\mathcal{S} / \mathcal{S}^{\mu}$, which is represented by $\sum_{i} r_{\mu, i}\left[K_{\mu, i}\right]$, should be zero by the observation. Therefore, $r_{\mu, i}=0$ for all $i$ and $\mu \neq \lambda$, by the conclusion of Theorem B that the classes $\left[K_{\mu, i}\right]$ are linearly independent in $\mathcal{S} / \mathcal{S}^{\mu}$. This proves the claim.

Fix $\lambda \in \Lambda$ and temporarily denote by $\mathbb{Z}^{\infty}$ the free abelian group generated by the collection $\left\{K_{\lambda, i}\right\}_{i}$. The assignment $K_{\lambda, i} \mapsto\left[K_{\lambda, i}\right]$ gives rise to an epimorphism $\mathbb{Z}^{\infty} \rightarrow \mathcal{S}_{\lambda} / \Delta$ by the claim. Because $\mathcal{S}$ is generated by $\left\{K_{\lambda, i}\right\}_{\lambda, i}$ and $\left[K_{\mu, i}\right]=0$ in $\mathcal{S} / \mathcal{S}^{\lambda}$ for $\mu \neq \lambda$, the composition

$$
\mathbb{Z}^{\infty} \rightarrow \mathcal{S}_{\lambda} / \Delta \hookrightarrow \mathcal{S} / \Delta \rightarrow \mathcal{S} / \mathcal{S}^{\lambda}
$$

is surjective. Moreover, by the linear independence of $\left[K_{\lambda, i}\right]$ in $\mathcal{S} / \mathcal{S}^{\lambda},(1.4)$ is an isomorphism. From this, it follows that both $\mathbb{Z}^{\infty} \rightarrow \mathcal{S}_{\lambda} / \Delta$ and $\mathcal{S}_{\lambda} / \Delta \rightarrow \mathcal{S} / \mathcal{S}^{\lambda}$ are isomorphisms. This shows that Theorem A(1) holds.

Because (1.3) is zero for $\mu \neq \lambda \in \Lambda$, the composition of two horizontal arrows on the top row of (1.2) is the direct sum of the isomorphisms $\mathcal{S}_{\lambda} / \Delta \rightarrow \mathcal{S} / \mathcal{S}^{\lambda}$. So the top row composition in (1.2) is an isomorphism. Also, the first horizontal arrow in the top row is surjective by the definition of $\mathcal{S}$. From this, it follows that Theorem A(2) and (3) hold.

\subsection{Primary decomposition for the bipolar filtration}

The method of this article provides further information on the structure of $\mathcal{T}$. To discuss this, we consider the bipolar filtration of $\mathcal{T}$, which was defined by Cochran, Harvey and Horn [11]. It is a descending filtration

$$
\{0\} \subset \cdots \subset \mathcal{T}_{n} \subset \cdots \subset \mathcal{T}_{1} \subset \mathcal{T}_{0} \subset \mathcal{T}
$$

where the subgroup $\mathcal{T}_{n}$ consists of concordance classes of certain knots called $n$-bipolar (see Definition 2.1). It is known that various modern smooth invariants vanish for knots in the subgroups $\mathcal{T}_{0}$ and $\mathcal{T}_{1}$, but the associated graded groups $\operatorname{gr}_{n}(\mathcal{T}):=\mathcal{T}_{n} / \mathcal{T}_{n+1}$ are nontrivial for all $n$ [11, 6]. Indeed, the abelian group $\operatorname{gr}_{n}(\mathcal{T})$ is known to have infinite rank for $n=0$ [15] and for all $n \geq 2$ [6].

As an attempt to understand the structure of the filtration, we formulate and study the primary decomposition of $\operatorname{gr}_{n}(\mathcal{T})$. For an $n$-bipolar knot $K$, denote its class $\operatorname{in}_{\operatorname{gr}_{n}}(\mathcal{T})$ by $[K]$. Similar to the case of $\mathcal{T}$, for an irreducible element $\lambda$ in $\mathbb{Q}\left[t^{ \pm 1}\right]$, consider the following subgroups of $\operatorname{gr}_{n}(\mathcal{T})$ :

$$
\begin{aligned}
& \operatorname{gr}_{n}(\mathcal{T})_{\lambda}:=\left\{[K] \in \operatorname{gr}_{n}(\mathcal{T}) \mid \Delta_{K}=\left(\lambda \lambda^{*}\right)^{k} \text { for some } k \geq 0\right\}, \\
& \operatorname{gr}_{n}(\mathcal{T})^{\lambda}:=\left\{[K] \in \operatorname{gr}_{n}(\mathcal{T}) \mid \Delta_{K} \text { is relatively prime to } \lambda\right\}
\end{aligned}
$$


Also, let $\operatorname{gr}_{n}(\Delta)=\left\{[K] \in \operatorname{gr}_{n}(\mathcal{T}) \mid \Delta_{K}\right.$ is trivial $\}$. The injections $\operatorname{gr}_{n}(\mathcal{T})_{\lambda} / \operatorname{gr}_{n}(\Delta) \hookrightarrow \operatorname{gr}_{n}(\mathcal{T}) / \operatorname{gr}_{n}(\Delta)$ and the surjections $\operatorname{gr}_{n}(\mathcal{T}) / \operatorname{gr}_{n}(\Delta) \rightarrow \operatorname{gr}_{n}(\mathcal{T}) / \operatorname{gr}_{n}(\mathcal{T})^{\lambda}$ induce homomorphisms

$$
\begin{aligned}
& \Phi_{L}^{n}: \bigoplus_{[\lambda]} \operatorname{gr}_{n}(\mathcal{T})_{\lambda} / \operatorname{gr}_{n}(\Delta) \longrightarrow \operatorname{gr}_{n}(\mathcal{T}) / \operatorname{gr}_{n}(\Delta), \\
& \Phi_{R}^{n}: \operatorname{gr}_{n}(\mathcal{T}) / \operatorname{gr}_{n}(\Delta) \longrightarrow \bigoplus_{[\lambda]} \operatorname{gr}_{n}(\mathcal{T}) / \operatorname{gr}_{n}(\mathcal{T})^{\lambda}
\end{aligned}
$$

The following is an analogue of Question 1.1.

Question 1.2 (Primary decomposition for the associated graded).

(1) Is $\Phi_{L}^{n}$ an isomorphism?

(2) Is $\Phi_{R}^{n}$ an isomorphism?

(3) Are $\operatorname{gr}_{n}(\mathcal{T})_{\lambda} / \operatorname{gr}_{n}(\Delta)$ and $\operatorname{gr}_{n}(\mathcal{T}) / \operatorname{gr}_{n}(\mathcal{T})^{\lambda}$ nontrivial for every irreducible $\lambda$ that arises as a factor of an Alexander polynomial of a knot?

(4) Is the following composition an isomorphism?

$$
\operatorname{gr}_{n}(\mathcal{T})_{\lambda} / \operatorname{gr}_{n}(\Delta) \hookrightarrow \operatorname{gr}_{n}(\mathcal{T}) / \operatorname{gr}_{n}(\Delta) \rightarrow \operatorname{gr}_{n}(\mathcal{T}) / \operatorname{gr}_{n}(\mathcal{T})^{\lambda}
$$

The following result supports affirmative answers. Similar to the case of $\mathcal{T}$, for a subgroup $\mathcal{S}$ in $\operatorname{gr}_{n}(\mathcal{T})$, let $\mathcal{S}_{\lambda}=\mathcal{S} \cap \operatorname{gr}_{n}(\mathcal{T})_{\lambda}$ and $\mathcal{S}^{\lambda}=\mathcal{S} \cap \operatorname{gr}_{n}(\mathcal{T})^{\lambda}$. Recall that the collection $\Lambda$ has been defined in (1.1) above.

Theorem C. Let $n \geq 2$. Then there is a subgroup $\mathcal{S}$ in $\operatorname{gr}_{n}(\mathcal{T})$ that contains $\operatorname{gr}_{n}(\Delta)$ such that

$$
\bigoplus_{\lambda \in \Lambda} \mathcal{S}_{\lambda} / \operatorname{gr}_{n}(\Delta) \stackrel{\cong}{\rightarrow} \mathcal{S} / \operatorname{gr}_{n}(\Delta) \stackrel{\cong}{\rightarrow} \bigoplus_{\lambda \in \Lambda} \mathcal{S} / \mathcal{S}^{\lambda}
$$

and $\mathcal{S}_{\lambda} / \operatorname{gr}_{n}(\Delta) \cong \mathbb{Z}^{\infty} \cong \mathcal{S} / \mathcal{S}^{\lambda}$ for every $\lambda \in \Lambda$.

By the same argument as the proof that Theorem $\mathrm{A}$ is equivalent to Theorem $\mathrm{B}$, it is seen that Theorem $\mathrm{C}$ is equivalent to the following statement.

Theorem D. Let $n \geq 2$. Then for each $\lambda \in \Lambda$, there are infinitely many topologically slice $n$-bipolar knots $K_{\lambda, i}(i=1,2, \ldots)$ with $\Delta_{K_{\lambda, i}}$ a power of $\lambda \lambda^{*}$ that are linearly independent in $\operatorname{gr}_{n}(\mathcal{T}) / \mathrm{gr}_{n}(\mathcal{T})^{\lambda}$.

Also, Theorem D implies Theorem B. Indeed, if a linear combination $\#_{i} a_{i} K_{\lambda, i}$ of the knots $K_{\lambda, i}$ in Theorem D is smoothly concordant to a knot $L$ with $\Delta_{L}$ relatively prime to $\lambda$, then the class $[L]$ automatically lies in the subgroup $\mathcal{T}_{n}$ of $\mathcal{T}$ because so are $K_{\lambda, i}$, and thus $[L]$ is zero in the quotient $\operatorname{gr}_{n}(\mathcal{T}) / \operatorname{gr}_{n}(\mathcal{T})^{\lambda}$. It follows that $a_{i}=0$ for all $i$, by Theorem $\mathrm{D}$. Therefore, to obtain Theorems A, B and $\mathrm{C}$, it suffices to prove Theorem D.

The remaining part of this article is organised as follows. Sections 2-4 are devoted to the proof of Theorem D. In the appendix, we discuss a general formulation of the notion of primary decomposition.

\subsubsection{Ingredients of the proof}

The proof of the above results uses (the ideas of) several results in the literature. To extract obstructions to smooth concordance, we combine the Cheeger-Gromov $L^{2} \rho$-invariants or, equivalently, $L^{2}$ signature defects and the Ozsváth-Szabó $d$-invariant defined from Heegaard Floer homology, following the approach of [6], which was motivated by earlier work of Cochran, Harvey and Horn [11]. The amenable signature theorem developed in [8,3] and Ozsváth-Szabó's $d$-invariant inequality for definite 4-manifolds [40] are among the key ingredients. We develop and use a localisation technique inspired by work of Cochran, Harvey and Leidy [14, 13], to produce representations of the fundamental group to which our $\rho$-invariants are associated. Also, to compute and estimate $d$-invariants of infinitely many branched covers of the infinitely many topologically slice knots in Theorems B and D, we use Némethi's work [38] on Heegaard Floer homology of negative definite plumbed 3-manifolds. 


\section{The first step of the proof of Theorem $D$}

As a preliminary of the proof of Theorem D, we recall the definition of the bipolar filtration from [11]. Let $M(K)$ be the zero framed surgery manifold of a knot $K$ in $S^{3}$.

Definition 2.1 ([11, Definition 5.1]). Let $n \geq 0$ be an integer. A compact connected 4-manifold $V$ bounded by $M(K)$ is an $n$-negaton if the following are satisfied:

(1) The inclusion induces an isomorphism on $H_{1}(M(K)) \rightarrow H_{1}(V)$, and a meridian of $K$ normally generates $\pi_{1}(V)$.

(2) There are disjointly embedded closed connected surfaces $S_{i}$ in $V$ that form a basis for $H_{2}(V)$ and have self-intersection -1 or, equivalently, the normal bundle of $S_{i}$ has Euler class -1 .

(3) For each $i$, the image of $\pi_{1}\left(S_{i}\right)$ lies in the $n$th derived subgroup $\pi_{1}(V)^{(n)}$.

If there is an $n$-negaton bounded by $M(K)$, then $K$ is called $n$-negative. An $n$-positon and an $n$-positive knot are defined by replacing -1 by +1 in condition (2) above. A knot $K$ is $n$-bipolar if $K$ is $n$-positive and $n$-negative.

Recall that $\mathcal{T}$ is the smooth concordance group of topologically slice knots. The bipolar filtration $\left\{\mathcal{T}_{n}\right\}_{n \geq 0}$ of $\mathcal{T}$ is defined by

$$
\mathcal{T}_{n}=\{[K] \in \mathcal{T} \mid K \text { is } n \text {-bipolar }\} .
$$

Because an $(n+1)$-bipolar knot is $n$-bipolar, $\left\{\mathcal{T}_{n}\right\}_{n \geq 0}$ is a descending filtration. It is an open problem whether $\bigcap_{n \geq 0} \mathcal{T}_{n}=\{0\}$.

\subsection{Construction of a family of knots $\left\{K_{i}\right\}$}

We start the proof of Theorem D with a construction of knots that will be shown to generate the promised infinite rank free abelian subgroup.

Fix an integer $n \geq 2$, as in Theorem D. Also, fix another integer $m \geq 1$. Several objects we will use below depend on $(n, m)$, but we omit it from notation because $(m, n)$ is fixed in our arguments. Let

$$
\lambda(t)=\lambda_{m}(t)=(m+1) t-m \in \Lambda .
$$

For each $(n, m)$, we will construct an infinite family of knots $\left\{K_{i}\right\}$ indexed by integers $i>0$, whose Alexander polynomial is $\lambda(t) \lambda\left(t^{-1}\right)$. The construction is similar to [6, Section 2.2]. (See also [11], which influenced the construction of [6] and ours.) Let $R(J, D)$ be the knot shown in Figure 1. Here $J$ and $D$ are knots that will be specified later. (For now, ignore the circles $\alpha_{J}$ and $\alpha_{D}$.) The knot $R(J, D)$ bounds an obviously seen Seifert surface of genus one, which consists of a 0-handle and two 1-handles. In Figure 1, the two 1-handles are untwisted and cross each other $2 m+1$ times. So, $S=\left[\begin{array}{cc}0 & m+1 \\ m & 0\end{array}\right]$ is a Seifert matrix. We remark that [6] and [11] use the particular case of $m=1$.

Because $t S-S^{T}$ presents the Alexander module of $R(J, D)$, a routine computation shows that $R(J, D)$ has (integral) Alexander module

$$
H_{1}\left(M(R(J, D)) ; \mathbb{Z}\left[t^{ \pm 1}\right]\right)=\mathbb{Z}\left[t^{ \pm 1}\right] /\langle\lambda(t)\rangle \oplus \mathbb{Z}\left[t^{ \pm 1}\right] /\left\langle\lambda\left(t^{-1}\right)\right\rangle,
$$

and the summands $\mathbb{Z}\left[t^{ \pm 1}\right] /\langle\lambda(t)\rangle$ and $\mathbb{Z}\left[t^{ \pm 1}\right] /\left\langle\lambda\left(t^{-1}\right)\right\rangle$ are equal to the subgroups $\left\langle\alpha_{J}\right\rangle$ and $\left\langle\alpha_{D}\right\rangle$ generated by the loops $\alpha_{J}$ and $\alpha_{D}$ shown in Figure 1. It follows that $\Delta_{R(J, D)}=\lambda(t) \lambda\left(t^{-1}\right)$. Moreover, the Blanchfield pairing

$$
B \ell: \mathcal{A} \times \mathcal{A} \longrightarrow \mathbb{Q}(t) / \mathbb{Q}\left[t^{ \pm 1}\right]
$$

on the Alexander module $\mathcal{A}:=H_{1}\left(M(R(J, D)) ; \mathbb{Q}\left[t^{ \pm 1}\right]\right)$ has exactly two metabolisers, $\left\langle\alpha_{J}\right\rangle$ and $\left\langle\alpha_{D}\right\rangle$. Here, a submodule $P \subset \mathcal{A}$ is called a metaboliser if $P$ is equal to $P^{\perp}:=\{x \in \mathcal{A} \mid B \ell(P, x)=0\}$. The above observations on $R(J, D)$ hold for any choice of $J$ and $D$. 


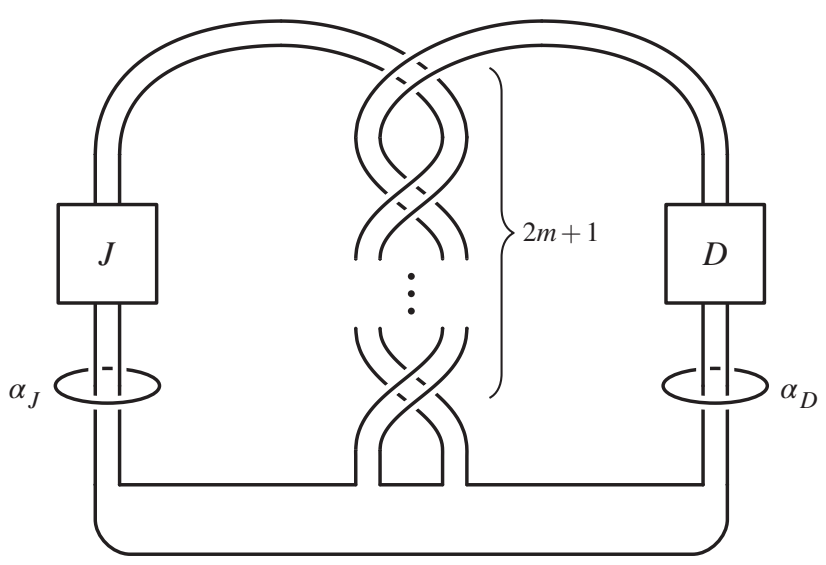

Figure 1. The knot $R(J, D)$.

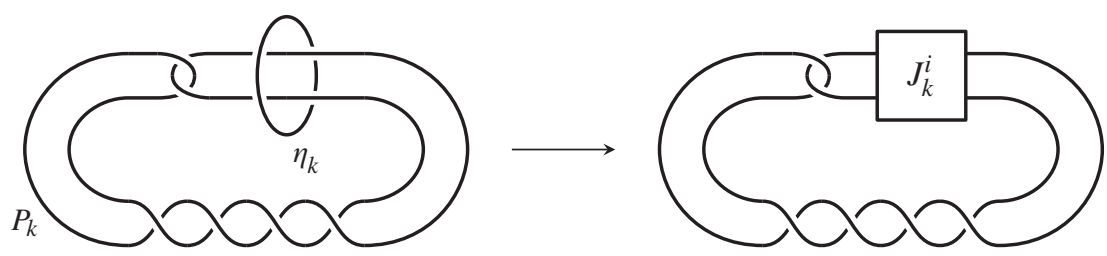

Figure 2. The stevedore's pattern $\left(P_{k}, \eta_{k}\right)$ and the satellite knot $P_{k}\left(\eta_{k}, J_{k}^{i}\right)$.

As another ingredient of the construction of the promised knots $K_{i}$, we will use a family of knots $\left\{J_{k}^{i}\right\}$, which was given in [6]. For $k=0$, an explicit construction of such $\left\{J_{0}^{i}\right\}_{i}$ is given in [6, Section 4]. We need that $\left\{J_{0}^{i}\right\}_{i}$ satisfies the following conditions (J1), (J2) and (J3) for some sequence of increasing primes $\left\{p_{i}\right\}$. For a knot $J$ and an integer $p$, let $\sigma_{J}(\omega) \in \mathbb{Z}$ be the Levine-Tristram signature function of $J$ at $\omega \in S^{1} \subset \mathbb{C}$, and let

$$
\rho\left(J, \mathbb{Z}_{d}\right)=\frac{1}{d} \sum_{k=0}^{d-1} \sigma_{J}\left(e^{2 \pi k \sqrt{-1} / d}\right) .
$$

(J1) For each $i, J_{0}^{i}$ is 0 -negative.

(J2) For each $i,\left|\rho\left(J_{0}^{i}, \mathbb{Z}_{p_{i}}\right)\right|>69713280 \cdot(6 n+8 m+86)$.

(J3) For $i<j, \rho\left(J_{0}^{j}, \mathbb{Z}_{p_{i}}\right)=0$.

For $k=0, \ldots, n-2, J_{k+1}^{i}$ is defined inductively by $J_{k+1}^{i}=P_{k}\left(\eta_{k}, J_{k}^{i}\right)$, where $P_{k}\left(\eta_{k}, J_{k}^{i}\right)$ is the satellite knot shown in the right of Figure 2. The left of Figure 2 shows the pattern $P_{k}$ in the exterior of an unknotted circle $\eta_{k}$, which is a standard solid torus. The companion is the knot $J_{k}^{i}$.

Now, let $K_{i}=R\left(J_{n-1}^{i}, D\right)$, where $D=\mathrm{Wh}^{+}(T)$ is the positive Whitehead double of the right-handed trefoil $T$. Because $D$ is topologically slice [21], $K_{i}$ is topologically concordant to $R\left(J_{n-1}^{i}, U\right)$, where $U$ is the trivial knot. Because $R(J, U)$ is (smoothly) slice for any $J$, it follows that $K_{i}$ is topologically slice. By [6, Section 2.2, Lemma 2.3], property (J1) implies that each $K_{i}$ is $n$-negative and $k$-positive for all $k \geq 0$. Because $\Delta_{K_{i}}=\lambda(t) \lambda\left(t^{-1}\right)$, it follows that the class $\left[K_{i}\right]$ lies in $\operatorname{gr}_{n}(\mathcal{T})_{\lambda}=\left(\mathcal{T}_{n} \cap \mathcal{T}_{\lambda}\right) /\left(\mathcal{T}_{n+1} \cap \mathcal{T}_{\lambda}\right)$. Therefore, to prove Theorem D, it suffices to show the following statement.

Theorem 2.2. Suppose that $K=\left(\#_{i=1}^{r} a_{i} K_{i}\right) \# L\left(a_{i} \in \mathbb{Z}\right)$ is a linear combination of the knots $K_{i}$ and $a$ knot $L$ with $\Delta_{L}(t)$ relatively prime to $\lambda(t)$. If $a_{i} \neq 0$ for some $i$, then $K$ is not $(n+1)$-bipolar. 
For the special case that $L$ is a trivial knot and $m=1$, the conclusion of Theorem 2.2 was shown in [6]. The general case of Theorem 2.2 requires substantially more sophisticated ideas and methods, which will be discussed in Sections 3 and 4.

\subsection{Construction of a negaton}

To prove Theorem 2.2 by contradiction, suppose that the knot $K$ is $(n+1)$-bipolar. Recall that $K_{i}$ is $n$-bipolar. The following observation will be useful. The knot $L$ in the statement of Theorem 2.2 is automatically $n$-bipolar, because $L$ is concordant to the sum of $K,-a_{1} K_{1}, \ldots-a_{r-1} K_{r-1}$, and $-a_{r} K_{r}$ and each of $K$ and $K_{i}$ are $n$-bipolar.

We may assume $a_{i} \neq 0$ for all $i$, by removing $K_{i}$ when $a_{i}=0$. In addition, by taking $-K$ instead of $K$, we may assume that $a_{1}>0$. Under this assumption, we will prove that $K$ is not $(n+1)$-negative. To derive a contradiction, suppose that $K$ is $(n+1)$-negative. As done in [6, Section 2.3], we will construct a certain $n$-negaton for the first summand $K_{1}$, which we will call $X^{-}$below. Because the generality of Theorem 2.2 does not cause significant issues in this construction, we will closely follow [6], with minor additional changes related to $L$.

Use the $n$-bipolarity of $L$ to choose an $n$-negaton, say, $Z_{L}^{-}$, bounded by $-M(L)=M(-L)$. Use the $n$-bipolarity of $K_{i}$, choose an $n$-negaton $Z_{i}^{-}$bounded by $-M\left(K_{i}\right)=M\left(-K_{i}\right)$ for each $i$ for which $a_{i}>0$ and choose $n$-negaton $Z_{i}^{-}$bounded by $M\left(K_{i}\right)$ for each $i$ for which $a_{i}<0$. Let $V^{-}$be an $(n+1)$-negaton bounded by $M(K)$. There is a standard cobordism $C$ bounded by the union of $\partial_{-} C:=-M(K)$ and $\partial_{+} C:=$ $\left(\bigsqcup_{i} a_{i} M\left(K_{i}\right)\right) \sqcup M(L)$, which is associated with the connected sum expression $K=\left(\#_{i=1}^{r} a_{i} K_{i}\right) \# L ; C$ is obtained by attaching to $\left(\bigsqcup_{i=1}^{r} a_{i} M\left(K_{i}\right) \times I\right) \sqcup M(L) \times I, N$ 1-handles that connect the component and then attaching $N$ 2-handles that make meridians of the involved $N+1$ knots parallel, where $N=\sum_{i}\left|a_{i}\right|$. A detailed description of $C$ can be found, for instance, from [17, p. 113]. Define

$$
X^{-}:=V^{-} \underset{\partial_{-} C}{\cup} C \underset{\partial_{+} C}{\cup}\left(\left(a_{1}-1\right) Z_{1}^{-} \sqcup\left(\bigsqcup_{i>1}\left|a_{i}\right| Z_{i}^{-}\right) \sqcup Z_{L}^{-}\right) .
$$

Figure 3 depicts the construction of $X^{-}$. By (the argument of) [6, Lemma 2.4], $X^{-}$is an $n$-negaton bounded by $M\left(K_{1}\right)$.

Now, let

$$
P:=\operatorname{Ker}\left\{H_{1}\left(M\left(K_{1}\right) ; \mathbb{Q}\left[t^{ \pm 1}\right]\right) \rightarrow H_{1}\left(X^{-} ; \mathbb{Q}\left[t^{ \pm 1}\right]\right)\right\} .
$$

Because $X^{-}$is an $n$-negaton with $n \geq 2, P$ is a metaboliser of the Blanchfield pairing on $H_{1}\left(M\left(K_{1}\right) ; \mathbb{Q}\left[t^{ \pm 1}\right]\right)$, by [11, Theorem 5.8]. (See also the statement of [6, Lemma 2.5].) Because $K_{1}=$ $R\left(J_{n-1}^{1}, D\right)$ has exactly two metabolisers $\left\langle\alpha_{J}\right\rangle$ and $\left\langle\alpha_{D}\right\rangle$, we have the following two cases: $P=\left\langle\alpha_{D}\right\rangle$ or $P=\left\langle\alpha_{J}\right\rangle$. By deriving a contradiction for each case, the proof of Theorem 2.2 will be completed.

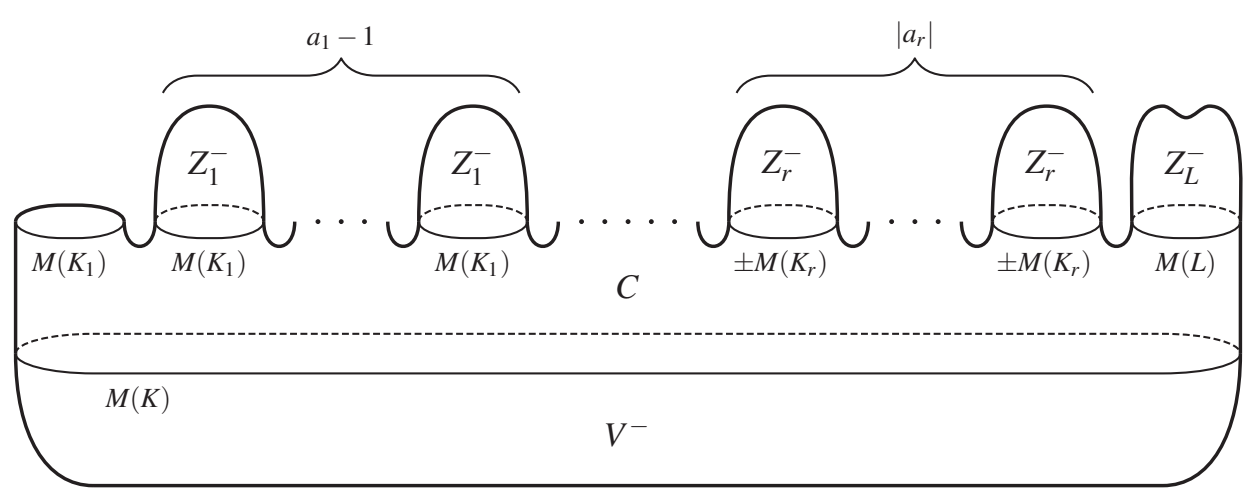

Figure 3. The construction of $X^{-}$. The sign of $M\left(K_{i}\right)$ equals that of $a_{i}$. 


\section{3. $L^{2}$-signatures and localised mixed-type commutator series}

In this section, we continue the proof of Theorem 2.2 for the case $P=\left\langle\alpha_{D}\right\rangle$.

Recall that we constructed an $n$-negaton $X^{-}$in (2.3) using the negatons $V^{-}, Z_{L}^{-}$and $Z_{i}^{-}$. To obtain a hyperbolic intersection form, take the connected sum of the $(n+1)$-negaton $V^{-}$and $b_{2}\left(V^{-}\right)$copies of $\mathbb{C} P^{2}$ and call the result $V^{0}$. Indeed, by [11, Proposition 5.5], $V^{0}$ is a special type of 4-manifold called an integral $(n+1)$-solution in [3, Definition 3.1], which particularly has a metabolic intersection form over twisted coefficients. We do not describe its definition because we do not use it directly, but we will state and use its properties later. For $Z_{L}^{-}$and $Z_{i}^{-}$, define integral $n$-solutions $Z_{L}^{0}$ and $Z_{i}^{0}$ by taking connected sum with copies of $\mathbb{C} P^{2}$ in the same way.

Repeat the construction of $X^{-}$but now use $V^{0}, Z_{L}^{0}$ and $Z_{i}^{0}$ in place of $V^{-}, Z_{L}^{-}$and $Z_{i}^{-}$to obtain a 4-manifold $X^{0}$. By the proof of [6, Lemma 2.4], $b_{2}\left(X^{-}\right)$is equal to the sum of $b_{2}\left(V^{-}\right), b_{2}\left(Z_{L}^{-}\right)$, $\left(a_{1}-1\right) b_{2}\left(Z_{1}^{-}\right)$and $\left|a_{i}\right| b_{2}\left(Z_{i}^{-}\right)(i>1)$. Thus, $X^{0}=X^{-} \#\left(b_{2}\left(X^{-}\right) \mathbb{C} P^{2}\right)$. Because $X^{-}$is an $n$-negaton, it follows that $X^{0}$ is an integral $n$-solution, again by [11, Proposition 5.5].

We will attach additional pieces to $X^{0}$ to obtain a sequence of 4-manifolds, essentially following a technique that first appeared in [12]; see also [14, 13, 3, 6]. The notation used below is close to [3, 6]. Consider the satellite construction $J_{k+1}^{1}=P_{k}\left(\eta_{k}, J_{k}^{1}\right)$. Due to [12], there is a standard cobordism, which we denote by $E_{k}$, from $M\left(J_{k+1}^{1}\right)$ to $M\left(J_{k}^{1}\right) \sqcup M\left(P_{k}\right)$ for $k=0, \ldots, n-2$. In Subsection 3.1, we will use an alternative description given in Figure 4, which illustrates that $E_{k}$ is obtained from $M\left(J_{k+1}^{1}\right) \times[0,1]$ by attaching a 2-handle and a 3-handle: start with $M\left(J_{k+1}^{1}\right)=M\left(P_{k}\left(\eta_{k}, J_{k}^{1}\right)\right)$, attach a 2-handle along a zero-framed longitude of $J_{k}^{1}$ to obtain the second diagram and apply handle slide to obtain the last diagram, which is $M\left(J_{k}^{1}\right) \# M\left(P_{k}\right)$. Attach a 3-handle to obtain $M\left(J_{k}^{1}\right) \sqcup M\left(P_{k}\right)$.

View $K_{1}=R\left(J_{n-1}^{1}, D\right)$ as the satellite knot $R(U, D)\left(\alpha_{J}, J_{n-1}^{1}\right)$ and apply the same construction to obtain a standard cobordism $E_{n-1}$ from $M\left(K_{1}\right)$ to $M\left(J_{n-1}^{1}\right) \sqcup M(R(U, D))$. Now, define 4-manifolds $X_{n}, X_{n-1}, \ldots, X_{0}=X$ as follows:

$$
\begin{aligned}
X_{n} & :=X^{0} \\
X_{n-1} & :=X_{n} \underset{M\left(K_{1}\right)}{\cup} E_{n-1}=X^{0} \underset{M\left(K_{1}\right)}{\cup} E_{n-1} \\
X_{n-2} & :=X_{n-1} \underset{M\left(J_{n-1}^{1}\right)}{\cup} E_{n-2}=X^{0} \underset{M\left(K_{1}\right)}{\cup} E_{n-1} \underset{M\left(J_{n-1}^{1}\right)}{\cup} E_{n-2} \\
& \vdots \\
X=X_{0} & :=X_{1} \underset{M\left(J_{1}^{1}\right)}{\cup} E_{0}=X^{0} \underset{M\left(K_{1}\right)}{\cup} E_{n-1} \underset{M\left(J_{n-1}^{1}\right)}{\cup} E_{n-2} \underset{M\left(J_{n-2}^{1}\right)}{\cup} \cdots \underset{M\left(J_{1}^{1}\right)}{\cup} E_{0} .
\end{aligned}
$$

See the schematic diagram in Figure 5.
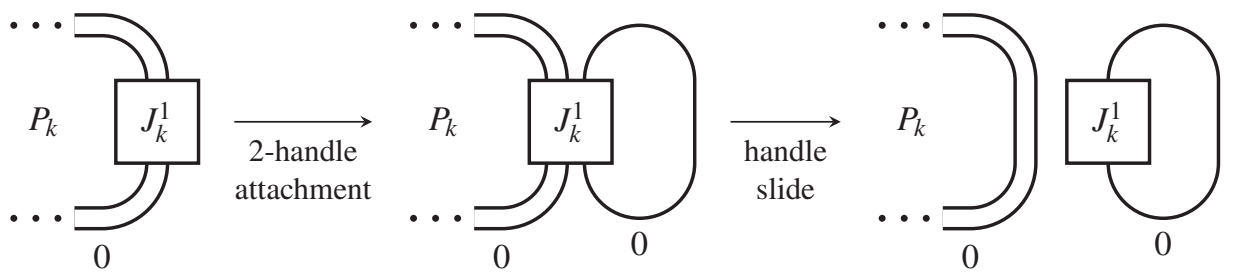

Figure 4. A handlebody description of the standard cobordism $E_{k}$. 


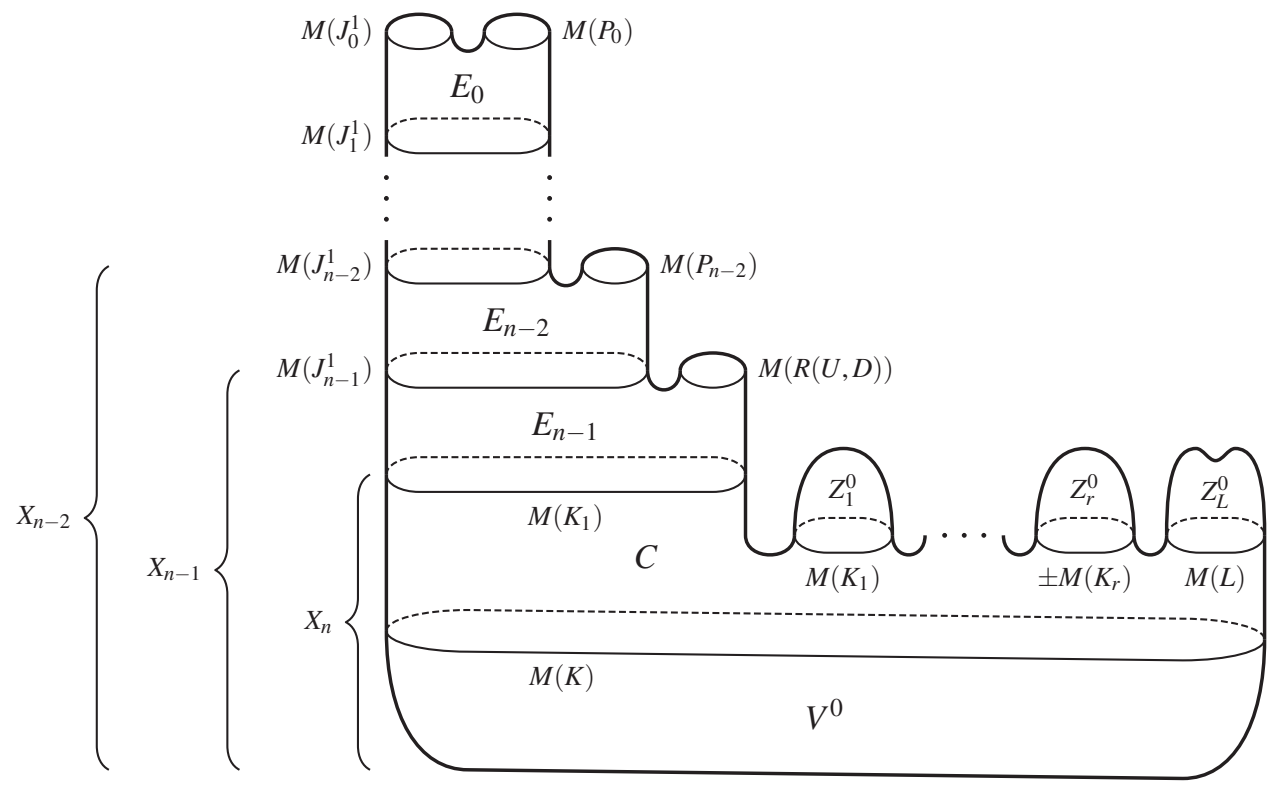

Figure 5. A schematic diagram of the 4-manifold X.

We have that $H_{1}\left(X^{0}\right)=H_{1}\left(X^{-}\right)=\mathbb{Z}=\langle t\rangle$ where the generator $t$ is represented by the meridian $\mu_{K}$ of $K$, because $X^{-}$is a negaton bounded by $M(K)$. See Definition 2.3(1). By a Mayer-Vietoris argument using this, it follows that $H_{1}\left(X_{k}\right)=\mathbb{Z}$ for all $k=0, \ldots, n$.

\subsection{A localised mixed-type commutator series associated with $X$}

Recall from Subsection 2.1 that $p_{1}$ denotes the first prime used in properties $(\mathrm{J} 2)$ and $(\mathrm{J} 3)$, and $\lambda(t)=$ $(m+1) t-m$. Let

$$
\Sigma:=\left\{f(t) \in \mathbb{Q}\left[t^{ \pm 1}\right] \mid f(1) \neq 0, \operatorname{gcd}\left(f(t), \lambda(t) \lambda\left(t^{-1}\right)\right)=1\right\}
$$

Obviously, $\Sigma$ is a multiplicative subset. Let $\mathbb{Q}\left[t^{ \pm}\right] \Sigma^{-1}$ be the localisation.

Definition 3.1. Let $G$ be a group endowed with a homomorphism $G \rightarrow \pi_{1}(X)$ that induces an epimorphism $H_{1}(G) \rightarrow H_{1}(X)$. For $i=0,1, \ldots, n+1$, define subgroups $\mathcal{P}^{i} G$ of $G$ inductively as follows. Let $\mathcal{P}^{0} G:=G$, and let $\mathcal{P}^{1} G$ be the kernel of the composition

$$
G \longrightarrow \pi_{1}(X) \longrightarrow H_{1}(X)=\mathbb{Z}=\langle t\rangle .
$$

Let $\mathcal{P}^{2} G$ be the kernel of the composition

$$
\begin{aligned}
\mathcal{P}^{1} G & \longrightarrow \frac{\mathcal{P}^{1} G}{\left[\mathcal{P}^{1} G, \mathcal{P}^{1} G\right]} \longrightarrow \frac{\mathcal{P}^{1} G}{\left[\mathcal{P}^{1} G, \mathcal{P}^{1} G\right] \mathbb{Z}} \otimes \mathbb{Q}=H_{1}\left(G ; \mathbb{Q}\left[t^{ \pm 1}\right]\right) \longrightarrow H_{1}\left(G ; \mathbb{Q}\left[t^{ \pm 1}\right] \Sigma^{-1}\right) \\
& \longrightarrow H_{1}\left(X ; \mathbb{Q}\left[t^{ \pm 1}\right] \Sigma^{-1}\right) \longrightarrow H_{1}\left(X ; \mathbb{Q}\left[t^{ \pm 1}\right] \Sigma^{-1}\right) / \operatorname{Im} H_{1}\left(Z_{L}^{0} ; \mathbb{Q}\left[t^{ \pm 1}\right] \Sigma^{-1}\right) .
\end{aligned}
$$

Here, $\operatorname{Im} H_{1}\left(Z_{L}^{0} ; \mathbb{Q}\left[t^{ \pm 1}\right] \Sigma^{-1}\right)$ is the image of $H_{1}\left(Z_{L}^{0} ; \mathbb{Q}\left[t^{ \pm 1}\right] \Sigma^{-1}\right) \rightarrow H_{1}\left(X ; \mathbb{Q}\left[t^{ \pm 1}\right] \Sigma^{-1}\right)$ induced by the inclusion. 
For $i=2, \ldots, n-1$, let

$$
\mathcal{P}^{i+1} G:=\operatorname{Ker}\left\{\mathcal{P}^{i} G \rightarrow \frac{\mathcal{P}^{i} G}{\left[\mathcal{P}^{i} G, \mathcal{P}^{i} G\right]} \rightarrow \frac{\mathcal{P}^{i} G}{\left[\mathcal{P}^{i} G, \mathcal{P}^{i} G\right]} \underset{\mathbb{Z}}{\otimes} \mathbb{Q}=H_{1}\left(G ; \mathbb{Q}\left[G / \mathcal{P}^{i} G\right]\right)\right\} .
$$

Finally, define

$$
\mathcal{P}^{n+1} G:=\operatorname{Ker}\left\{\mathcal{P}^{n} G \rightarrow \frac{\mathcal{P}^{n} G}{\left[\mathcal{P}^{n} G, \mathcal{P}^{n} G\right]} \rightarrow \frac{\mathcal{P}^{n} G}{\left[\mathcal{P}^{n} G, \mathcal{P}^{n} G\right]} \underset{\mathbb{Z}}{\otimes \mathbb{Z}_{p_{1}}}=H_{1}\left(G ; \mathbb{Z}_{p_{1}}\left[G / \mathcal{P}^{n} G\right]\right)\right\} .
$$

It is straightforward to verify inductively that $\mathcal{P}^{k} G$ is a normal subgroup of $G$ and the standard $k$ th derived subgroup $G^{(k)}$ lies in $\mathcal{P}^{k} G$ for all $k$.

We remark that the commutator series $\left\{\mathcal{P}^{k} G\right\}_{k}$ in Definition 3.1 is 'localised at polynomials' in the sense of [14, Sections 3 and 4] and is of 'mixed-coefficient' type in the sense that we use both $\mathbb{Z}_{p_{1}}$ and $\mathbb{Q}$ (see [3, Section 4.1]).

In particular, define the subgroups $\mathcal{P}^{i} \pi_{1}\left(X_{k}\right)$, by applying Definition 3.1 to the case $G=\pi_{1}\left(X_{k}\right) \rightarrow$ $\pi_{1}(X)$. The following properties, which we will state as Assertions A and B, are essential for our purpose. Let $\mu_{k} \subset M\left(J_{k}^{1}\right)$ be the meridian of $J_{k}^{1}$. By the construction of $X_{k}, M\left(J_{k}^{1}\right)$ is a component of $\partial X_{k}$ (see Figure 5), and thus $\mu_{k}$ represents an element in $\pi_{1}\left(X_{k}\right)$ for $k \leq n-1$. For brevity, let $J_{n}^{1}:=K_{1}$, so that the previous sentence holds for $k=n$ as well. Also, let $\left(P_{n-1}, \eta_{n-1}\right):=\left(R(U, D), \alpha_{D}\right)$, so that $J_{k}^{1}=P_{k-1}\left(\eta_{k-1}, J_{k-1}^{1}\right)$ holds for $k=n$ as well.

Assertion A. The class of $\mu_{k}$ lies in $\pi_{1}\left(X_{k}\right)^{(n-k)} \subset \mathcal{P}^{n-k} \pi_{1}\left(X_{k}\right)$ and is nontrivial in $\mathcal{P}^{n-k} \pi_{1}\left(X_{k}\right) / \mathcal{P}^{n-k+1} \pi_{1}\left(X_{k}\right)$ for all $k=0,1, \ldots, n$. In particular, the class $\mu_{0}$ is nontrivial in $\mathcal{P}^{n} \pi_{1}(X) / \mathcal{P}^{n+1} \pi_{1}(X)$.

Assertion B. For $i=1, \ldots, n$, the inclusion-induced map sends $\pi_{1}\left(Z_{L}^{0}\right)^{(i)}$ to the subgroup $\mathcal{P}^{i+1} \pi_{1}(X) \subset$ $\pi_{1}(X)$. In particular, $\pi_{1}\left(Z_{L}^{0}\right)^{(n)}$ maps to $\mathcal{P}^{n+1} \pi_{1}(X)$.

\section{Remark 3.2.}

(1) Analogues of Assertion A for similar situations were established in earlier papers; for instance, in $[12,14,3,4,6]$. We will give a proof for our case, because our series $\mathcal{P}^{i}$ is different (notably at $i=2$ ) from those in the literature. Assertion B and its application are new, to the author's knowledge.

(2) Note that both $\mu_{0}$ and $\pi_{1}\left(Z_{L}^{0}\right)^{(n)}$ map to the $n$th subgroup $\mathcal{P}^{n} \pi_{1}(X)$. Due to Assertions A and B, they have opposite nature in the next stage: $\pi_{1}\left(Z_{L}^{0}\right)^{(n)}$ lies in $\mathcal{P}^{n+1} \pi_{1}(X)$, whereas $\mu_{0}$ does not. This will be crucial in separating the contribution of the unknown knot $L$ from that of $K_{1}$ in the linear combination $K=\left(\#_{i=1}^{r} a_{i} K_{i}\right) \# L$. See Subsection 3.2, particularly the Cheeger-Gromov $\rho$-invariants in (3.3) and (3.8).

In the proof of Assertion A, the following fact will be useful. Let $\lambda_{k-1}$ be the zero-linking longitude of $J_{k-1}^{1}$, which lies in

$$
E\left(J_{k-1}^{1}\right) \subset E\left(J_{k-1}^{1}\right) \cup E\left(P_{k-1} \sqcup \eta_{k-1}\right)=E\left(J_{k}^{1}\right) \subset M\left(J_{k}^{1}\right) \subset \partial X_{k} .
$$

Assertion C. The inclusion $X_{k} \subset X_{k-1}$ induces an isomorphism $\mathcal{P}^{i} \pi_{1}\left(X_{k}\right) /\left\langle\lambda_{k-1}\right\rangle \cong \mathcal{P}^{i} \pi_{1}\left(X_{k-1}\right)$ for all $i \leq n-k+2$. Consequently, we have

$$
\mathcal{P}^{n-k+1} \pi_{1}\left(X_{k}\right) / \mathcal{P}^{n-k+2} \pi_{1}\left(X_{k}\right) \cong \mathcal{P}^{n-k+1} \pi_{1}\left(X_{k-1}\right) / \mathcal{P}^{n-k+1} \pi_{1}\left(X_{k-1}\right) .
$$

Proof of Assertion $C$. Because $E_{k-1}$ is obtained by attaching a 2-handle to $M\left(J_{k}^{1}\right) \times[0,1]$ along $\lambda_{k-1}$ and then attaching a 3-handle (see Figure 4), $X_{k-1}=X_{k} \cup E_{k-1}$ is obtained from $X_{k}$ by the same handle attachments. It follows that $\pi_{1}\left(X_{k-1}\right) \cong \pi_{1}\left(X_{k}\right) /\left\langle\lambda_{k-1}\right\rangle$. This shows the assertion for $i=0$. Now, to 
proceed by induction, suppose that $i \geq 1$ and suppose that the assertion holds for $i-1$. If $i=1$, we have the following commutative diagram with exact rows:

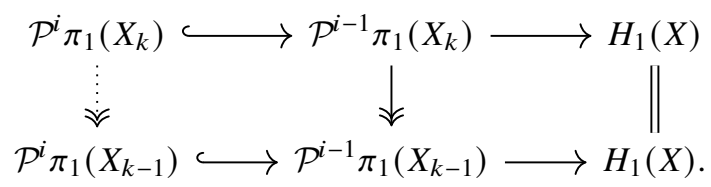

Because the two rightmost horizontal arrows are to the same target $H_{1}(X)$, there is an induced epimorphism $\mathcal{P}^{i} \pi_{1}\left(X_{k}\right) \rightarrow \mathcal{P}^{i} \pi_{1}\left(X_{k-1}\right)$ and its kernel is equal to that of the epimorphism $\mathcal{P}^{i-1} \pi_{1}\left(X_{k}\right) \rightarrow$ $\mathcal{P}^{i-1} \pi_{1}\left(X_{k-1}\right)$. So the assertion holds for $i=1$. For $i=2$, replace $H_{1}(X)$ in the above diagram by the quotient

$$
H_{1}\left(X ; \mathbb{Q}\left[t^{ \pm 1}\right] \Sigma^{-1}\right) / \operatorname{Im} H_{1}\left(Z_{L}^{0} ; \mathbb{Q}\left[t^{ \pm 1}\right] \Sigma^{-1}\right)
$$

and apply the same argument.

For $i \geq 3$, we have

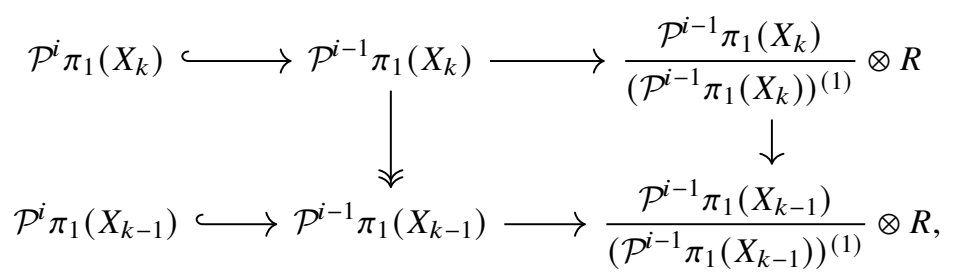

where $R=\mathbb{Q}$ or $\mathbb{Z}_{p_{1}}$, depending on $i$. We claim that the rightmost vertical arrow is an isomorphism. From the claim, it follows that the assertion holds for $i$, once again by the argument used above. To show the claim, let $\gamma_{k-1}$ be the meridian of $J_{k-1}^{1}$ in the exterior $E\left(J_{k-1}^{1}\right) \subset M\left(J_{k}^{1}\right) \subset \partial X_{k}$. Note that $\gamma_{k-1}$ is different from the meridian $\mu_{k-1} \subset M\left(J_{k-1}^{1}\right) \subset \partial X_{k-1}$ used in the statement of Assertion A, but $\gamma_{k-1}$ and $\mu_{k-1}$ are isotopic in the cobordism $E_{k-1}$. The meridian $\gamma_{k-1}$ is identified with the curve $\eta_{k-1}$, which lies in the commutator subgroup $\pi_{1}\left(M\left(J_{k}^{1}\right)\right)^{(1)}$. Because $\pi_{1}\left(M\left(J_{k}^{1}\right)\right)$ is normally generated by the meridian $\mu_{k}, \gamma_{k-1}$ lies in $\left\langle\mu_{k}\right\rangle^{(1)}=\left\langle\gamma_{k}\right\rangle^{(1)}$ in $\pi_{1}\left(X_{k}\right)$. By induction, it follows that $\gamma_{k-1}$ lies in $\left\langle\gamma_{n}\right\rangle^{(n-k+1)}$. Therefore, the image of $\pi_{1}\left(E\left(J_{k-1}^{1}\right)\right)$ lies in $\pi_{1}\left(X_{k}\right)^{(n-k+1)}$. Because the longitude $\lambda_{k-1}$ lies in $\pi_{1}\left(E\left(J_{k-1}^{1}\right)\right)^{(1)}$, it follows that

$$
\lambda_{k-1} \in \pi_{1}\left(X_{k}\right)^{(n-k+2)} .
$$

Because $i \leq n-k+2$, (3.1) implies that $\lambda_{k-1} \in \pi_{1}\left(X_{k}\right)^{(i)} \subset\left(\mathcal{P}^{i-1} \pi_{1}\left(X_{k}\right)\right)^{(1)}$. Also, by the induction hypothesis, $\mathcal{P}^{i-1} \pi_{1}\left(X_{k}\right) \rightarrow \mathcal{P}^{i-1} \pi_{1}\left(X_{k-1}\right)$ is an epimorphism with kernel $\left\langle\lambda_{k-1}\right\rangle$. It follows that the rightmost vertical arrow in the above diagram is an isomorphism. This completes the proof of the assertion.

Proof of Assertion A. In the proof of Assertion C, we already showed that $\mu_{k}$ lies in $\pi_{1}\left(X_{k}\right)^{(n-k)}$. This is the first part of Assertion A.

It remains to show that $\mu_{k}$ is nontrivial in $\mathcal{P}^{n-k} \pi_{1}\left(X_{k}\right) / \mathcal{P}^{n-k+1} \pi_{1}\left(X_{k}\right)$. We will use reverse induction for $k=n, n-1, \ldots, 0$. For $k=n, \pi_{1}\left(X_{n}\right) / \mathcal{P}^{1} \pi_{1}\left(X_{n}\right)=H_{1}(X)$ by definition, and the meridian $\mu_{n}$ of $J_{n}^{1}=K_{1}$ is a generator of $H_{1}(X)$. So the assertion holds. 
For the case $k=n-1$, let $\Lambda=\mathbb{Q}\left[t^{ \pm 1}\right]$ for brevity, and consider the following commutative diagram:

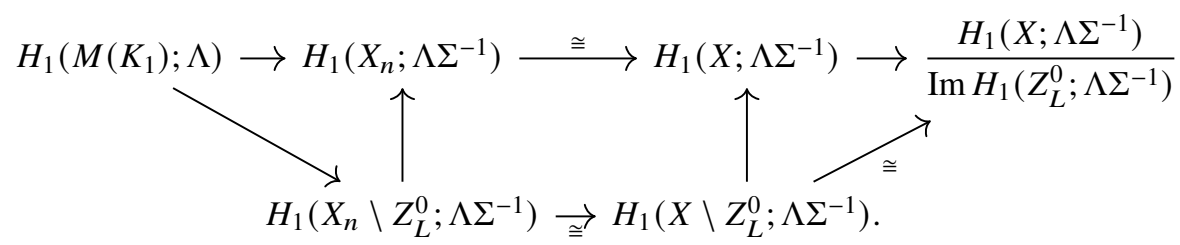

Here, $H_{1}\left(X_{n} ; \Lambda \Sigma^{-1}\right) \rightarrow H_{1}\left(X ; \Lambda \Sigma^{-1}\right)$ is an isomorphism, because $\pi_{1}(X)=\pi_{1}\left(X_{0}\right)$ is isomorphic to $\pi_{1}\left(X_{n}\right) /\left\langle\lambda_{0}, \ldots, \lambda_{n-1}\right\rangle$ by Assertion $C$, and the longitudes $\lambda_{0}, \ldots, \lambda_{n-1}$ lie in $\pi_{1}\left(X_{n}\right)^{(2)}$ by (3.1). The same argument shows that the bottom horizontal arrow is an isomorphism. (Alternatively, one may use Mayer-Vietoris arguments to show that they are isomorphisms.) Also, consider the Mayer-Vietoris sequence for $X=\overline{X \backslash Z_{L}^{0}} \cup_{M(L)} Z_{L}^{0}$ :

$$
H_{1}\left(M(L) ; \Lambda \Sigma^{-1}\right) \longrightarrow H_{1}\left(X \backslash Z_{L}^{0} ; \Lambda \Sigma^{-1}\right) \oplus H_{1}\left(Z_{L}^{0} ; \Lambda \Sigma^{-1}\right) \longrightarrow H_{1}\left(X ; \Lambda \Sigma^{-1}\right) \longrightarrow 0 .
$$

We have $H_{1}\left(M(L) ; \Lambda \Sigma^{-1}\right)=0$ by the hypothesis that $\Delta_{L}(t)$ is relatively prime to the polynomial $\lambda(t)$. It follows that the diagonal arrow on the right-hand side of the above diagram is an isomorphism.

By our hypothesis, the kernel $P$ of

$$
\left\langle\alpha_{J}\right\rangle \oplus\left\langle\alpha_{D}\right\rangle=H_{1}\left(M\left(K_{1}\right) ; \Lambda\right) \longrightarrow H_{1}\left(X_{n} ; \Lambda\right)
$$

is equal to the summand $\left\langle\alpha_{D}\right\rangle$. So the other summand $\left\langle\alpha_{J}\right\rangle$ injects into $H_{1}\left(X_{n} ; \Lambda\right)$. Because $\left\langle\alpha_{J}\right\rangle \cong$ $\Lambda /\langle\lambda\rangle$ is not annihilated by $\Sigma$, this implies that $\left\langle\alpha_{J}\right\rangle$ injects into $H_{1}\left(X_{n} ; \Lambda \Sigma^{-1}\right)=H_{1}\left(X_{n} ; \Lambda\right) \Sigma^{-1}$. By the above diagram, it follows that $\left\langle\alpha_{J}\right\rangle$ injects into $H_{1}\left(X \backslash Z_{L}^{0} ; \Lambda \Sigma^{-1}\right)$. Thus, $\alpha_{J}$ is nontrivial in $H_{1}\left(X ; \Lambda \Sigma^{-1}\right) / \operatorname{Im} H_{1}\left(Z_{L}^{0} ; \Lambda \Sigma^{-1}\right)$. Therefore, by the definition of $\mathcal{P}^{2} \pi_{1}\left(X_{n}\right), \alpha_{J}$ is nontrivial in the quotient

$$
\mathcal{P}^{1} \pi_{1}\left(X_{n}\right) / \mathcal{P}^{2} \pi_{1}\left(X_{n}\right) \subset H_{1}\left(X ; \Lambda \Sigma^{-1}\right) / \operatorname{Im} H_{1}\left(Z_{L}^{0} ; \Lambda \Sigma^{-1}\right) .
$$

By Assertion $\mathrm{C}, \mathcal{P}^{1} \pi_{1}\left(X_{n}\right) / \mathcal{P}^{2} \pi_{1}\left(X_{n}\right) \cong \mathcal{P}^{1} \pi_{1}\left(X_{n-1}\right) / \mathcal{P}^{2} \pi_{1}\left(X_{n-1}\right)$. Also, $\alpha_{J}$ is isotopic to the meridian $\mu_{n-1}$ in $X_{n-1}$. It follows that $\mu_{n-1}$ is nontrivial in the quotient $\mathcal{P}^{1} \pi_{1}\left(X_{n-1}\right) / \mathcal{P}^{2} \pi_{1}\left(X_{n-1}\right)$. This is exactly the promised conclusion for $k=n-1$.

Now, suppose $0 \leq k \leq n-2$. The induction hypothesis is that $\mu_{k+1}$ is nontrivial in the quotient $\mathcal{P}^{n-k-1} \pi_{1}\left(X_{k+1}\right) / \mathcal{P}^{n-k} \pi_{1}\left(X_{k+1}\right)$. To show that $\mu_{k}$ is nontrivial in $\mathcal{P}^{n-k} \pi_{1}\left(X_{k}\right) / \mathcal{P}^{n-k+1} \pi_{1}\left(X_{k}\right)$, we use an argument that is essentially the same as [3, Proof of Theorem 4.14], which was influenced by [12]. Let $R=\mathbb{Q}$ if $k \geq 1$, and $R=\mathbb{Z}_{p_{1}}$ if $k=0$. Let

$$
B: H_{1}\left(M\left(J_{k+1}^{1}\right) ; R\left[t^{ \pm 1}\right]\right) \times H_{1}\left(M\left(J_{k+1}^{1}\right) ; R\left[t^{ \pm 1}\right]\right) \longrightarrow R(t) / R\left[t^{ \pm 1}\right]
$$

be the classical Blanchfield pairing of $J_{k+1}^{1}$ over $R$-coefficients. For brevity, let $G=$ $\pi_{1}\left(X_{k+1}\right) / \mathcal{P}^{n-k} \pi_{1}\left(X_{k+1}\right)$. Consider the noncommutative Alexander module $\mathcal{A}:=H_{1}\left(M\left(J_{k+1}^{1}\right) ; R G\right)$. The noncommutative Blanchfield pairing $\mathcal{B}: \mathcal{A} \times \mathcal{A} \rightarrow \mathcal{K} / R G$ is defined following [16, Theorem 2.13], where $\mathcal{K}$ is the skew-quotient field of $R G$. (For $R=\mathbb{Z}_{p_{1}}$, see also [3, Section 5].) In our case, because $2 \leq n-k \leq n$, from Definition 3.1 it follows that $G=\pi_{1}\left(X_{k+1}\right) / \mathcal{P}^{n-k} \pi_{1}\left(X_{k+1}\right)$ is poly-torsion-freeabelian and, consequently, $R G$ is an Ore domain due to [16, Proposition 2.5] and [3, Lemma 5.2]. We will use the following known facts:

(1) The nontriviality of $\mu_{k+1}$ in $\mathcal{P}^{n-k-1} \pi_{1}\left(X_{k+1}\right) / \mathcal{P}^{n-k} \pi_{1}\left(X_{k+1}\right) \subset G$ implies that $\mathcal{A} \cong$ $R G \otimes_{R\left[t^{ \pm 1}\right]} H_{1}\left(M\left(J_{k+1}^{1}\right) ; R\left[t^{ \pm 1}\right]\right)$ and that $\mathcal{B}(1 \otimes x, 1 \otimes y)=0$ if and only if $B(x, y)=0$. This 
is due to [33, Theorem 4.7], [2, Theorem 5.16] and [12, Lemma 6.5, Theorem 6.6]. See also [3, Theorem 5.4].

(2) The 4-manifold $X_{k+1}$ endowed with $\pi_{1}\left(X_{k+1}\right) \rightarrow G$ is a Blanchfield bordism in the sense of [4, Definition 4.11], due to an argument in [4, p. 3270] that uses [4, Theorem 4.13]. (The 4-manifold $W_{k+1}$ in [4] plays the role of our $X_{k+1}$.) The only property of a Blanchfield bordism we need is the following: for all $z$ in $\operatorname{Ker}\left\{\mathcal{A} \rightarrow H_{1}\left(X_{k+1} ; R G\right)\right\}, \mathcal{B}(z, z)=0$ by [4, Theorem 4.12].

Recall that $E\left(P_{k} \sqcup \eta_{k}\right) \cup E\left(J_{k}^{1}\right)=E\left(J_{k+1}^{1}\right) \subset M\left(J_{k+1}^{1}\right) \subset \partial X_{k+1}$. Denote a zero-linking longitude of $\eta_{k}$ in $E\left(P_{k} \sqcup \eta_{k}\right) \subset M\left(J_{k+1}^{1}\right)$ by $\eta_{k}$, abusing notation. If $\eta_{k}$ is trivial in $\mathcal{P}^{n-k} \pi_{1}\left(X_{k+1}\right) / \mathcal{P}^{n-k+1} \pi_{1}\left(X_{k+1}\right)$, then by the definition of $\mathcal{P}^{n-k+1}, \eta_{k}=1 \otimes \eta_{k}$ lies in the kernel of $\mathcal{A} \rightarrow H_{1}\left(X_{k+1} ; R G\right)$. By (2), from this it follows that $\mathcal{B}\left(1 \otimes \eta_{k}, 1 \otimes \eta_{k}\right)=0$ and, consequently, by $(1), B\left(\eta_{k}, \eta_{k}\right)=0$. Because $J_{k+1}^{1}=P_{k}\left(\eta_{k}, J_{k}^{1}\right)$ with $k \leq n-2$, the Alexander module $H_{1}\left(M\left(J_{k+1}^{1}\right) ; R\left[t^{ \pm 1}\right]\right)$ is isomorphic to that of stevedore's knot $P_{k}$, which is a cyclic module generated by $\eta_{k}$. It contradicts the nonsingularity of the classical Blanchfield pairing $B$. This shows that $\eta_{k}$ is nontrivial in $\mathcal{P}^{n-k} \pi_{1}\left(X_{k+1}\right) / \mathcal{P}^{n-k+1} \pi_{1}\left(X_{k+1}\right)$, which is isomorphic to $\mathcal{P}^{n-k} \pi_{1}\left(X_{k}\right) / \mathcal{P}^{n-k+1} \pi_{1}\left(X_{k}\right)$ by Assertion C. Because $\eta_{k}$ is identified with $\mu_{k}$, it follows that $\mu_{k}$ is nontrivial in the quotient $\mathcal{P}^{n-k} \pi_{1}\left(X_{k}\right) / \mathcal{P}^{n-k+1} \pi_{1}\left(X_{k}\right)$. This completes the proof of Assertion A.

Proof of Assertion B. Recall that Assertion B says that $\pi_{1}\left(Z_{0}^{L}\right)^{(i)}$ maps to $\mathcal{P}^{i+1} \pi_{1}(X)$ for $1 \leq i \leq n$. To show this for $i=1$, observe that the composition

$$
\begin{aligned}
\frac{\pi_{1}\left(Z_{L}^{0}\right)^{(1)}}{\pi_{1}\left(Z_{L}^{0}\right)^{(2)}}=H_{1}\left(Z_{L}^{0} ; \mathbb{Z}\left[t^{ \pm 1}\right]\right) & \longrightarrow H_{1}\left(X ; \mathbb{Z}\left[t^{ \pm 1}\right]\right) \longrightarrow H_{1}\left(X ; \mathbb{Q}\left[t^{ \pm 1}\right] \Sigma^{-1}\right) \\
& \longrightarrow H_{1}\left(X ; \mathbb{Q}\left[t^{ \pm 1}\right] \Sigma^{-1}\right) / \operatorname{Im} H_{1}\left(Z_{L}^{0} ; \mathbb{Q}\left[t^{ \pm 1}\right] \Sigma^{-1}\right)
\end{aligned}
$$

is obviously zero. By definition of $\mathcal{P}^{2} \pi_{1}(X)$, it follows that $\pi_{1}\left(Z_{L}^{0}\right)^{(1)}$ maps to $\mathcal{P}^{2} \pi_{1}(X)$. Therefore, for all $1 \leq i \leq n, \pi_{1}\left(Z_{L}^{0}\right)^{(i)}=\left(\pi_{1}\left(Z_{L}^{0}\right)^{(1)}\right)^{(i-1)}$ maps to $\mathcal{P}^{2} \pi_{1}(X)^{(i-1)}$, which is a subgroup of $\mathcal{P}^{i+1} \pi_{1}(X)$.

\subsection{Obstruction from Cheeger-Gromov $\rho$-invariants}

Now, we will use the Cheeger-Gromov $\rho$-invariant to derive a contradiction. We begin with some background. For a connected closed 3-manifold $M$ and a homomorphism $\phi: \pi_{1}(M) \rightarrow \Gamma$ with $\Gamma$ arbitrary, the Cheeger-Gromov invariant $\rho^{(2)}(M, \phi) \in \mathbb{R}$ is defined [10]. The value of $\rho^{(2)}(M, \phi)$ is preserved under composition with automorphisms of $\pi_{1}(M)$, so one can view $\rho^{(2)}(M, \phi)$ as an invariant of $M$ equipped with (the homotopy class of) a map $\phi: M \rightarrow B \Gamma=K(\Gamma, 1)$, instead of $\pi_{1}(M) \rightarrow \Gamma$. Even when $M$ is not connected, $\rho^{(2)}(M, \phi)$ is defined for $\phi: M \rightarrow B \Gamma$, with additivity under disjoint union: $\rho^{(2)}(M, \phi)=\sum_{i} \rho^{(2)}\left(M_{i},\left.\phi\right|_{M_{i}}\right)$ where $M=\bigcup_{i} M_{i}$ with components $M_{i}$.

In this article, we do not use the definition of $\rho^{(2)}\left(M, \rho^{(2)}\right)$ given in [10]. Instead, for our purpose, the following $L^{2}$-signature defect interpretation is useful. If $M=\cup M_{i}$ bounds a 4-manifold $W$ and $\phi: M \rightarrow B \Gamma$ factors through $W$, then $\rho^{(2)}(M, \phi)=\sum_{i} \rho^{(2)}\left(M_{i},\left.\phi\right|_{M_{i}}\right)$ is equal to the $L^{2}$-signature defect $\bar{\sigma}_{\Gamma}^{(2)}(W):=\operatorname{sign}_{\Gamma}^{(2)}(W)-\operatorname{sign}(W)$, where $\operatorname{sign}(W)$ is the ordinary signature and $\operatorname{sign}_{\Gamma}^{(2)}(W)$ is the $L^{2}$ signature of $W$ over the group $\Gamma$. We note that this approach can also be used to provide an alternative definition of $\rho^{(2)}(M, \phi)$ for arbitrary $(M, \phi)$. As references, see, for instance, [9], [18, Section 2], [23, Section 3], [5, Section 2.1].

For our case, let $\Gamma:=\pi_{1}(X) / \mathcal{P}^{n+1} \pi_{1}(X)$. For a connected 3-dimensional submanifold $M$ in $X$, denote the composition $\pi_{1}(M) \rightarrow \pi_{1}(X) \rightarrow \Gamma$ by $\phi$, abusing notation. 
Then, by the $L^{2}$-signature defect interpretation for $\rho^{(2)}(\partial X, \phi)$, we have

$$
\begin{gathered}
\rho^{(2)}\left(M\left(J_{0}^{1}\right), \phi\right)+\sum_{k=0}^{n-2} \rho^{(2)}\left(M\left(P_{k}\right), \phi\right)+\rho^{(2)}(M(R(U, D)), \phi)=\bar{\sigma}_{\Gamma}^{(2)}(X) \\
=\bar{\sigma}_{\Gamma}^{(2)}\left(V^{0}\right)+\bar{\sigma}_{\Gamma}^{(2)}(C)+\bar{\sigma}_{\Gamma}^{(2)}\left(Z_{L}^{0}\right)+\sum_{i, j} \bar{\sigma}_{\Gamma}^{(2)}\left(Z_{i, j}^{0}\right)+\sum_{k=0}^{n-1} \bar{\sigma}_{\Gamma}^{(2)}\left(E_{k}\right),
\end{gathered}
$$

where the 4-manifolds $Z_{i, j}^{0}$ are copies of $Z_{i}^{0}$ used in the construction of $X$. (See Figure 5.) The second equality is obtained by Novikov additivity of $L^{2}$-signatures (for instance, see [16 Lemma 5.9]).

Recall that $\mu_{0}$ is the meridian of $J_{0}^{1}$ in $M\left(J_{0}^{1}\right)$. By Assertion A, $\phi\left(\mu_{0}\right)$ is nontrivial in $\Gamma$. Because $\phi\left(\mu_{J_{0}^{1}}\right)$ lies in the subgroup $\mathcal{P}^{n} \pi_{1}(X) / \mathcal{P}^{n+1} \pi_{1}(X)$ of $\Gamma$, which is a vector space over $\mathbb{Z}_{p_{1}}$ by Definition 3.1, it follows that $\mu_{J_{0}^{1}}$ has order $p_{1}$. So the image of $\pi_{1}\left(M\left(J_{0}^{1}\right)\right)$ in $\Gamma$ under $\phi$ is isomorphic to $\mathbb{Z}_{p_{1}}$. By [8, Lemma 8.7], this implies that

$$
\rho^{(2)}\left(M\left(J_{0}^{1}\right), \phi\right)=\rho\left(J_{0}^{1}, \mathbb{Z}_{p_{1}}\right),
$$

where $\rho\left(J_{0}^{1}, \mathbb{Z}_{p_{1}}\right)$ is defined by (2.2).

By the explicit universal bound for the Cheeger-Gromov invariants given in [5, Theorem 1.9], we have

$$
\left|\rho^{(2)}\left(M\left(P_{k}\right), \phi\right)\right| \leq 6 \cdot 69713280
$$

because stevedore's knot $P_{k}$ has six crossings. Similarly, by [5, Theorem 1.9],

$$
\left|\rho^{(2)}(M(R(U, D)), \phi)\right| \leq(8 m+92) \cdot 69713280
$$

because $R(U, D)$ has a diagram with $8 m+92$ crossings (see Figure 1 ).

By [12, Lemma 2.4], the following holds for each $k$.

$$
\bar{\sigma}_{G}^{(2)}(C)=0, \quad \bar{\sigma}_{G}^{(2)}\left(E_{k}\right)=0 .
$$

To evaluate the terms $\bar{\sigma}_{\Gamma}^{(2)}\left(V^{0}\right)$ and $\bar{\sigma}_{\Gamma}^{(2)}\left(Z_{L}^{0}\right)$ in (3.2), we will use the following result.

Theorem 3.3 (Amenable Signature Theorem [3, Theorem 3.2]). Suppose that $W$ is an integral $(n+1)$ solution bounded by the zero surgery manifold $M(K)$ of a knot $K$. Suppose that $\Gamma$ is a group that satisfies $\Gamma^{(n+1)}=\{1\}$ and lies in Strebel's class $D\left(\mathbb{Z}_{p_{1}}\right)$ in the sense of [45] (or, equivalently, $\Gamma$ is a locally $p$-indicable group, due to [26]). If $\phi: \pi_{1}(M(K)) \rightarrow \Gamma$ is a homomorphism that factors through $\pi_{1}(W)$ and sends the meridian of $K$ to an infinite-order element in $\Gamma$, then $\rho^{(2)}(M(K), \phi)=\bar{\sigma}_{\Gamma}^{(2)}(W)=0$.

In our case, $V^{0}$ is an integral $(n+1)$-solution bounded by $M(K)$. Also, the group $\Gamma$ lies in $D\left(\mathbb{Z}_{p_{1}}\right)$ by [8, Lemma 6.8], and we have $\Gamma^{(n+1)}=\{1\}$ because $\pi_{1}(X)^{(n+1)} \subset \mathcal{P}^{n+1} \pi_{1}(X)$. The meridian of $K$ has infinite order in $\Gamma$ because $\Gamma$ surjects onto $H_{1}(X)=\mathbb{Z}$ generated by the meridian. By Amenable Signature Theorem 3.3, it follows that

$$
\bar{\sigma}_{\Gamma}^{(2)}\left(V^{0}\right)=\rho^{(2)}(M(K), \phi)=0 .
$$

Now we will evaluate $\bar{\sigma}_{\Gamma}^{(2)}\left(Z_{L}^{0}\right)$, using the Amenable Signature Theorem again. Note that $\partial Z_{L}^{0}=$ $M(L)$. An important difference from the above paragraph is that the 4-manifold $Z_{L}^{0}$ is an integral $n$ solution, not $n+1$. So, the Amenable Signature Theorem does not apply directly over $\Gamma$, because $\Gamma^{(n)}$ is not necessarily trivial. Instead, we proceed as follows. 
Note that the map $\phi: \pi_{1}(M(L)) \rightarrow \pi_{1}\left(Z_{L}^{0}\right) \rightarrow \Gamma$ factors through $\pi_{1}\left(Z_{L}^{0}\right) / \pi_{1}\left(Z_{L}^{0}\right)^{(n)}$, by Assertion B. Let $G$ be the image of $\pi_{1}\left(Z_{L}^{0}\right) / \pi_{1}\left(Z_{L}^{0}\right)^{(n)}$ in $\Gamma$, and let $\psi: \pi_{1}(M(L)) \rightarrow G$ be the map induced by $\phi$. Because $G$ injects into $\Gamma$, we have $\rho^{(2)}(M(L), \phi)=\rho^{(2)}(M(L), \psi)$, by the $L^{2}$-induction property (for instance, see [10, Eq. 2.3]). Now, because $G$ is a subgroup of $\Gamma$ that is in $D\left(\mathbb{Z}_{p_{1}}\right), G$ is in Strebel's class $D\left(\mathbb{Z}_{p_{1}}\right)$, too. Also, $G^{(n)}$ is trivial because it is the image of $\pi_{1}\left(Z_{L}^{0}\right) / \pi_{1}\left(Z_{L}^{0}\right)^{(n)}$. The meridian $\mu_{L}$ of $L$ has infinite order in $G$, because $G$ surjects onto $H_{1}(X)=\mathbb{Z}$, which $\mu_{L}$ generates. Therefore, Amenable Signature Theorem 3.3 (with $n$ in place of $n+1$ ) applies to $(M(L), \psi)$ to conclude that

$$
\bar{\sigma}_{\Gamma}^{(2)}\left(Z_{L}^{0}\right)=\rho^{(2)}(M(L), \phi)=\rho^{(2)}(M(L), \psi)=0 .
$$

By [6, Lemma 3.3], we may assume that each $Z_{i, j}^{0}$ has the property that $\bar{\sigma}_{\Gamma}^{(2)}\left(Z_{i, j}^{0}\right)$ is equal to either 0 or $\rho\left(J_{0}^{i}, \mathbb{Z}_{p_{1}}\right)$. (Indeed, [6, Lemma 3.3] applies when every $J_{0}^{i}$ is 0 -negative; it is the case by property (J1) in Subsection 2.1.) Moreover, by property (J3) in Subsection 2.1, we have

$$
\bar{\sigma}_{\Gamma}^{(2)}\left(Z_{i, j}^{0}\right)= \begin{cases}0 \text { or } \rho\left(J_{0}^{1}, \mathbb{Z}_{p_{1}}\right) & \text { if } i=1 \\ 0 & \text { if } i>1\end{cases}
$$

Now, combine (3.2)-(3.9) to obtain

$$
N \cdot\left|\rho^{(2)}\left(J_{0}^{1}, \mathbb{Z}_{p_{1}}\right)\right| \leq(6 n+8 m+86) \cdot 69713280,
$$

where $N$ is one plus the number of the 4-manifolds $Z_{1, j}^{0}$ such that $\bar{\sigma}^{(2)}\left(Z_{1, j}^{0}\right) \neq 0$. Because $N \geq 1$, it contradicts property (J2) in Subsection 2.1. This completes the proof that $P$ cannot be equal to $\left\langle\alpha_{D}\right\rangle$. That is, $P$ must be $\left\langle\alpha_{J}\right\rangle$.

\section{Computing and estimating $d$-invariants}

In this section, we will continue the proof of Theorem 2.2, to reach a contradiction under the hypothesis that $P=\left\langle\alpha_{J}\right\rangle$.

\subsection{Finite cyclic covers and their d-invariants}

We begin by applying a trick introduced in [11], which we describe below. Let $K_{1}=K_{0}(\alpha, J)$ be a satellite knot, where the pattern satisfies $\operatorname{lk}\left(K_{0}, \alpha\right)=0$. Then, the identity on $E(K \sqcup \alpha)$ extends to a map $E\left(K_{1}\right)=E(K \sqcup \alpha) \cup E(J) \rightarrow E(K \sqcup \alpha) \cup E(U)=E\left(K_{0}\right)$ that induces an isomorphism $H_{1}\left(M\left(K_{1}\right) ; \mathbb{Q}\left[t^{ \pm 1}\right]\right) \rightarrow H_{1}\left(M\left(K_{0}\right) ; \mathbb{Q}\left[t^{ \pm 1}\right]\right)$, under which we will identify the Alexander modules. Essentially, [11, Lemma 8.2] says the following (see also [6, Lemma 5.1]): if $K_{1}=K_{0}(\alpha, J)$ admits a 1-negaton $X_{1}$ bounded by $M\left(K_{1}\right)$ and if $J$ is unknotted by changing some positive crossings to negative, then $K_{0}$ has a 1-negaton $X_{0}$ bounded by $M\left(K_{0}\right)$ such that the two maps

$$
H_{1}\left(M\left(K_{i}\right) ; \mathbb{Q}\left[t^{ \pm 1}\right]\right) \longrightarrow H_{1}\left(X_{i} ; \mathbb{Q}\left[t^{ \pm 1}\right]\right) \quad(i=0,1)
$$

have the identical kernel. In particular, this applies to the satellite knot $K_{1}=R(U, D)\left(\alpha_{D}, J_{n-1}^{1}\right)$ defined in Subsection 2.1, because $J_{n-1}^{1}=P_{n-2}\left(\eta_{n}, J_{n-2}^{1}\right)$ is unknotted by changing a single positive crossing (see Figure 2). Note that here we use that $n \geq 2$. Therefore, in our case, the knot $K_{0}:=R(U, D)$ admits a 1-negaton bounded by $M\left(K_{0}\right)$, say, $W$, such that

$$
\left\langle\alpha_{J}\right\rangle=\operatorname{Ker}\left\{H_{1}\left(M\left(K_{0}\right) ; \mathbb{Q}\left[t^{ \pm 1}\right]\right) \rightarrow H_{1}\left(W ; \mathbb{Q}\left[t^{ \pm 1}\right]\right)\right\} .
$$

We will derive a contradiction from the existence of this 1-negaton $W$ for $K_{0}$. 
The next step is to pass to finite-degree branched cyclic covers, to which Heegaard Floer homology machinery applies, following [6, Section 5.1]. Let $\Sigma_{r}$ be the $r$-fold branched cyclic cover of $\left(S^{3}, K_{0}\right)$. The curves $\alpha_{J}$ and $\alpha_{D}$ in Figure 1 represent homology classes in $\Sigma_{r}$, say, $x_{1}$ and $x_{2} \in H_{1}\left(\Sigma_{r}\right)$, respectively. (The classes $x_{1}$ and $x_{2}$ are defined up to covering transformation, but it will cause no problem in our argument.) Due to [37], we have $H_{1}\left(\Sigma_{r}\right)=H_{1}\left(M\left(K_{0}\right) ; \mathbb{Z}\left[t^{ \pm 1}\right]\right) /\left\langle t^{r}-1\right\rangle$. Recall that $H_{1}\left(M\left(K_{0}\right) ; \mathbb{Z}\left[t^{ \pm 1}\right]\right)$ is given by (2.1). From this, by elementary computation, it follows that $H_{1}\left(\Sigma_{r}\right)=$ $\mathbb{Z}_{(m+1)^{r}-m^{r}} \oplus \mathbb{Z}_{(m+1)^{r}-m^{r}}$ and the summands are generated by $x_{1}$ and $x_{2}$. In particular, $\Sigma_{r}$ is a $\mathbb{Z}_{2^{-}}$ homology sphere.

For a rational homology 3 -sphere $Y$ and a $\operatorname{spin}^{c}$ structure $\mathrm{t}$ on $Y$, Ozsváth and Szabó defined a correction term invariant $d(Y, \mathrm{t})$ using the Heegaard Floer chain complex [40]. In case of a $\mathbb{Z}_{2}$-homology sphere $Y$, the unique spin structure determines a canonical $\operatorname{spin}^{c}$ structure on $Y$, which we denote by $\mathfrak{s}_{Y}$, and all $\operatorname{spin}^{c}$ structures of $Y$ are given in the form $\mathfrak{s}_{Y}+c$, where $c \in H^{2}(Y)$ and + designates the action of $H^{2}(Y)$ on the set of $\operatorname{spin}^{c}$ structures. For $x \in H_{1}(Y)$, let $\widehat{x} \in H^{2}(Y)$ be the Poincare dual of $x$. Techniques used in $[6,11]$ give us the following $d$-invariant obstruction.

Lemma 4.1. Suppose that $M\left(K_{0}\right)$ bounds a 1-negaton that satisfies (4.1). If $r$ is sufficiently large, then $d\left(\Sigma_{r}, \mathfrak{s}_{\Sigma_{r}}+k \cdot \widehat{x_{1}}\right) \geq 0$ for all $k \in \mathbb{Z}$.

Proof. Attach a 2-handle to a given 1-negaton $W$, along the zero-framed meridian of $K_{0}$, to obtain a 4-manifold, which we temporarily call $V$. Note that $\partial V=S^{3}$ and the cocore of the 2-handle is a slicing disk $\Delta \subset V$ bounded by $K_{0}$. Take the $r$-fold branched cyclic cover of $(V, \Delta)$ and call it $V_{r}$. Here the $r$-fold branched cyclic cover is defined because, using that $W$ is a 1-negaton, we see that $H_{1}(W \backslash \Delta)=\mathbb{Z}$ generated by a meridian of $\Delta$. Indeed, if we denote by $W_{r}$ the $r$-fold cyclic cover of $W$, then $V_{r}$ is obtained by attaching a 2-handle to $W_{r}$. The 4-manifold $V_{r}$ is bounded by $\Sigma_{r}$.

The first key step is to relate the hypothesis $P=\left\langle\alpha_{J}\right\rangle$, which is associated with the infinite cyclic cover, to the kernel

$$
G:=\operatorname{Ker}\left\{H_{1}\left(\Sigma_{r}\right) \longrightarrow H_{1}\left(V_{r}\right)\right\}
$$

associated with finite covers. The following is a modified version of [6, Lemma 5.2].

Assertion. Under the hypothesis that $P=\left\langle\alpha_{J}\right\rangle, x_{1} \in G$ for all large primes $r$.

Although we do not use it, we remark that the assertion implies that $G=\left\langle x_{1}\right\rangle$, because it is known that $|G|$ is equal to $\left|H_{1}\left(\Sigma_{m}\right)\right|^{1 / 2}=(m+1)^{r}-m^{r}$.

Proof of Assertion. Let $E_{r}$ be the $r$-fold cyclic cover of the exterior $E\left(K_{0}\right)$ for $r \leq \infty$. Consider the following commutative diagram:

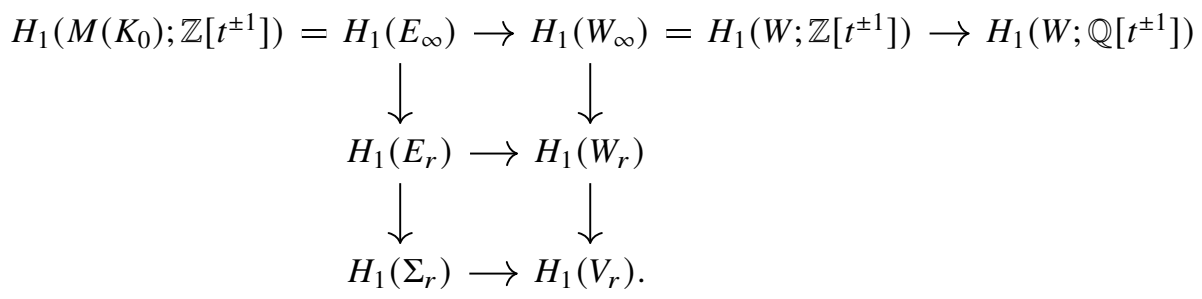

The vertical arrows are induced by coverings and inclusions. Because $\alpha_{J}$ lies in the kernel of the composition of the top row by the hypothesis, $\alpha_{J}$ is $\mathbb{Z}$-torsion in $H_{1}\left(W ; \mathbb{Z}\left[t^{ \pm 1}\right]\right)$. That is, $a \cdot \alpha_{j}=0$ in $H_{1}\left(W ; \mathbb{Z}\left[t^{ \pm 1}\right]\right)$ for some nonzero $a \in \mathbb{Z}$. By the above diagram, it follows that $a \cdot x_{1} \in H_{1}\left(\Sigma_{r}\right)$ lies in the kernel $G$ of $H_{1}\left(\Sigma_{r}\right) \rightarrow H_{1}\left(V_{r}\right)$.

Suppose that $r$ is a prime not smaller than any prime factor of $a$. Under this assumption, we claim that $\operatorname{gcd}\left(a,(m+1)^{r}-m^{r}\right)=1$. From this it follows that $x_{1}$ lies in $G$, because $x_{1}$ has order $(m+1)^{r}-m^{r}$ in $H_{1}\left(\Sigma_{r}\right)$. This proves the assertion, modulo the proof of the claim. 

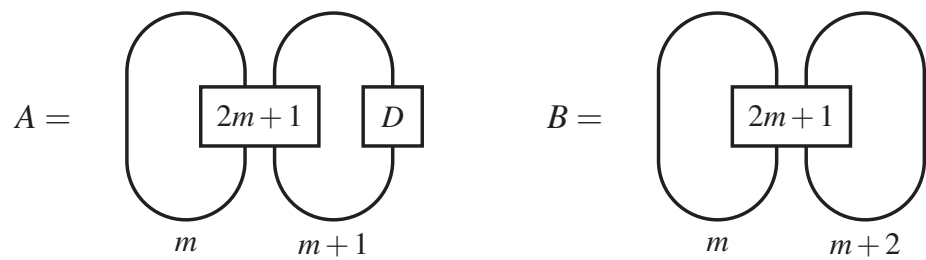

Figure 6. The 3-manifolds $A$ and B. The box $2 m+1$ represents $2 m+1$ right-handed full twists between vertical strands.

To show the claim, it suffices to show that every prime factor $q$ of $a$ is relatively prime to $(m+1)^{r}-m^{r}$. It is obviously true, if $q \mid m$ or $q \mid m+1$. So, suppose that $q$ divides neither $m$ nor $m+1$ but $q$ divides $(m+1)^{r}-m^{r}$. Let $u=m^{*}(m+1)$, where $m^{*}$ is an arithmetic inverse of $m \bmod q$. We have $u^{r} \equiv 1 \bmod q$ by the hypothesis and $u^{q-1} \equiv 1 \bmod q$ by Fermat's little theorem. Because $r$ is a prime and $u \neq \equiv 1 \bmod q$, it follows that $r \mid q-1$. This contradicts the assumption that $r \geq q$. This completes the proof of the claim.

The assertion enables us to invoke a result of Cochran, Harvey and Horn [11, Theorem 6.5], which says the following: if $W$ is a 1-negaton bounded by $M\left(K_{0}\right)$, then $d\left(\Sigma_{r}, \widehat{x}\right) \geq 0$ for all $x$ lying in $G=\operatorname{Ker}\left\{H_{1}\left(\Sigma_{r}\right) \rightarrow H_{1}\left(V_{r}\right)\right\}$. Applying this to $x=k \cdot x_{1}$, the proof of Lemma 4.1 is completed.

Theorem 4.2. Let $m \geq 1$ be an odd integer and $r \geq 1$ be an odd prime power. Let $k$ be the arithmetic inverse of $2 \bmod (m+1)^{r}-m^{r}$. Then $d\left(\Sigma_{r}, \mathfrak{s}_{\Sigma_{r}}+k \widehat{x}_{1}\right) \leq-\frac{3}{2}$.

On the other hand, Lemma 4.1 says that $d\left(\Sigma_{r}, \mathfrak{s}_{\Sigma_{r}}+k \widehat{x}_{1}\right)$ must be nonnegative. This contradiction implies that the kernel $P$ defined in (2.4) cannot be equal to $\left\langle\alpha_{J}\right\rangle$. This completes the proof of Theorem 2.2.

For $m=1$, Theorem 4.2 was already shown in [6, Theorem 5.4]. (We remark that the symbol $m$ in [6] denotes our $r$.) So, in the remaining part of this article, we will assume that $m>1$.

Proof of Theorem 4.2. Let $A$ and $B$ be the 3-manifolds given by the surgery presentations in Figure 6 . Let $Y_{r}$ be the connected sum of $A, B$ and $r-3$ copies of the lens space $L_{2 m+1,1}$. (We use the orientation convention that $L_{p, 1}$ is the $p$-framed surgery on the trivial knot $U$ in $S^{3}$.) Then, arguments in [6, Section 6.1] construct a negative definite 4-manifold $W$ with $\partial W=Y_{r} \sqcup-\Sigma_{r}$ and $b_{2}(W)=(2 m+1) r-$ $4 m+1$ and construct a $\operatorname{spin}^{c}$ structure $\mathrm{t}$ on $W$ such that $c_{1}(\mathrm{t})^{2}=-r, c_{1}\left(\left.\mathrm{t}\right|_{\Sigma_{r}}\right)=\widehat{x_{1}}$ and $c_{1}\left(\left.\mathrm{t}\right|_{Y_{r}}\right)=0$. Indeed, [6, Section 6.1] is the case of $m=1$. We do not repeat the details here, because exactly the same method works under our assumption that $m \geq 1$ is odd. Perhaps the least obvious part is that the inverse matrix $P^{-1}$ in [6, Eq. (6.10)] should be replaced with a block matrix $P^{-1}=\left(R_{i j}\right)_{1 \leq i, j \leq r-1}$ with

$$
R_{i j}=\frac{\left((m+1)^{i}-m^{i}\right)\left((m+1)^{r-j}-m^{r-j}\right)}{(m+1)^{r}-m^{r}}\left[\begin{array}{cc}
0 & (m+1)^{i-j} \\
m^{i-j} & 0
\end{array}\right] \text { for } i \geq j,
$$

and $R_{i j}=R_{j i}^{T}$ for $i<j$.

By applying Ozsváth-Szabó's $d$-invariant inequality [40, Theorem 9.6] to the negative definite 4manifold $W$, and by using additivity of the $d$-invariant under connected sum, we have

$$
\begin{aligned}
d\left(\Sigma_{r},\left.\mathrm{t}\right|_{\Sigma_{r}}\right) & \leq d\left(Y_{r},\left.\mathrm{t}\right|_{Y_{r}}\right)-\frac{c_{1}(\mathrm{t})^{2}+b_{2}(W)}{4} \\
& =d\left(A,\left.\mathrm{t}\right|_{A}\right)+d\left(B,\left.\mathrm{t}\right|_{B}\right)+(r-3) d\left(L_{2 m+1,1},\left.\mathrm{t}\right|_{L_{2 m+1,1}}\right)-\frac{2 m r-4 m+1}{4}
\end{aligned}
$$

Because $c_{1}\left(\left.\mathrm{t}\right|_{Y_{r}}\right)=0$, we have $\left.\right|_{L_{2 m+1,1}}=\mathfrak{s}_{L_{2 m+1,1}}$. By a $d$-invariant formula for lens spaces given in [40, Proposition 4.8], $d\left(L_{2 m+1,1}, \mathfrak{s}_{L_{2 m+1,1}}\right)=m / 2$. By Lemmas 4.3 and 4.4, which we will prove below, we have $d\left(A,\left.\mathrm{t}\right|_{A}\right) \leq(m-7) / 4$ and $d\left(B,\left.\mathrm{t}\right|_{B}\right) \leq(m+2) / 4$. Combine them with the above inequality to obtain 

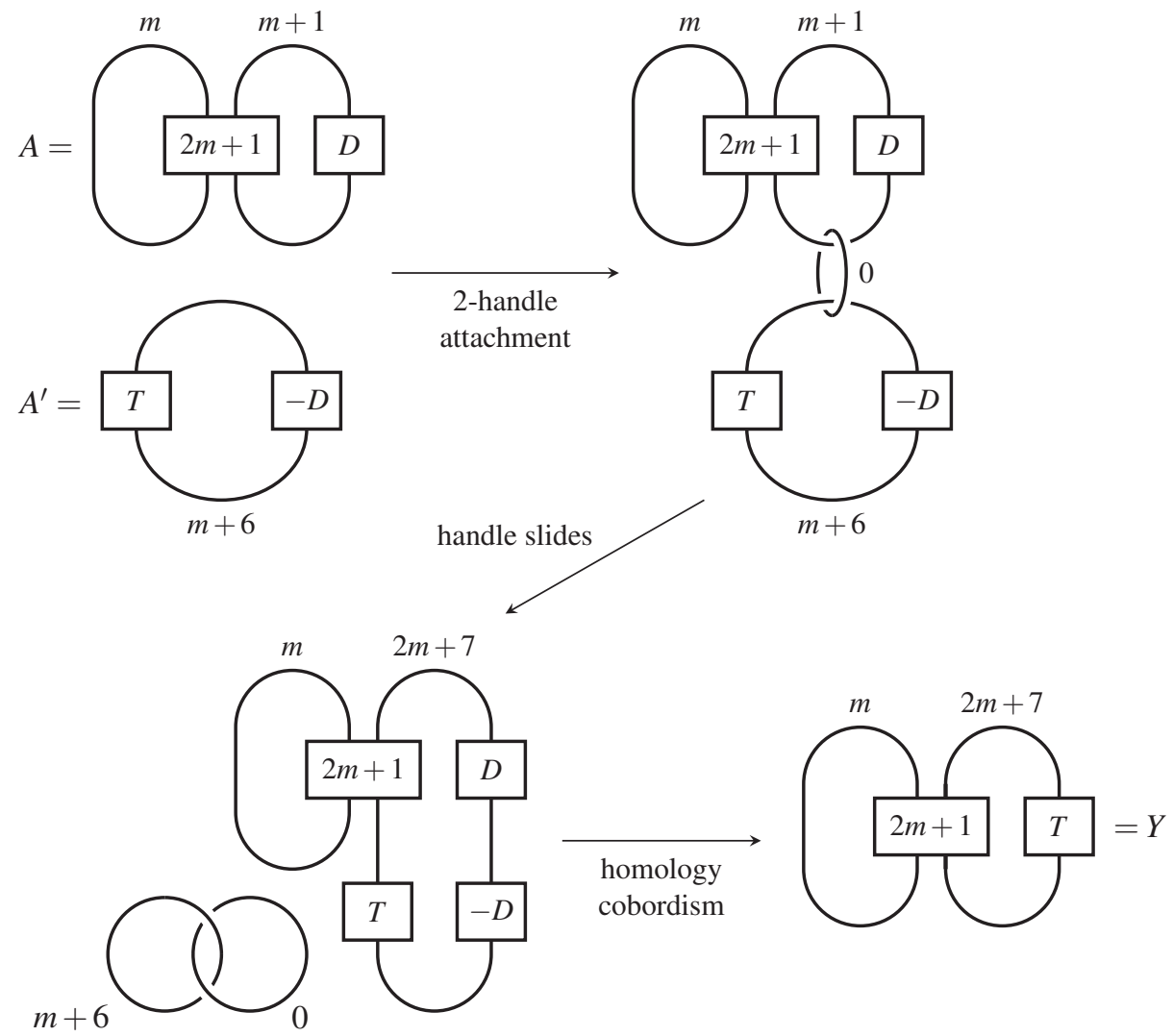

Figure 7. A cobordism from $A \# A^{\prime}$ to $Y$.

$d\left(\Sigma_{r},\left.\mathrm{t}\right|_{\Sigma_{r}}\right) \leq-\frac{3}{2}$. Observe that $c_{1}\left(\left.\mathrm{t}\right|_{\Sigma_{r}}\right)=\widehat{x_{1}}=2 k \widehat{x_{1}}=c_{1}\left(\mathfrak{s}_{\Sigma_{r}}+k \widehat{x_{1}}\right)$, because $2 k \equiv 1 \bmod (m+1)^{r}-m^{r}$ and $x_{1}$ has order $(m+1)^{r}-m^{r}$. It follows that $\mathrm{t}_{\Sigma_{r}}=\mathfrak{s}_{\Sigma_{r}}$, because $\Sigma_{r}$ is a $\mathbb{Z}_{2}$-homology sphere.

Note that $\left|H_{1}(A)\right|=\left|\operatorname{det}\left[\begin{array}{cc}m & -2 m-1 \\ -2 m-1 & m+1\end{array}\right]\right|=3 m^{2}+3 m+1$ is odd, so there is a unique $\operatorname{spin}^{c}$ structure $\mathfrak{s}$ of $A$ such that $c_{1}(\mathfrak{s})=0$.

Lemma 4.3. For the spin ${ }^{c}$ structure $\mathfrak{s}$ on $A$ with $c_{1}(\mathfrak{s})=0, d(A, \mathfrak{s}) \leq(m-7) / 4$.

For the case of $B$, because $\left|H_{1}(B)\right|=\left|\operatorname{det}\left[\begin{array}{cc}m & -2 m-1 \\ -2 m-1 & m+2\end{array}\right]\right|=3 m^{2}+2 m+1$ is even, there are exactly two $\operatorname{spin}^{c}$ structures $\mathfrak{s}$ of $B$ such that $c_{1}(\mathfrak{s})=0$.

Lemma 4.4. If $\mathfrak{s}$ is a spin ${ }^{c}$ structure of $B$ such that $c_{1}(\mathfrak{s})=0$, then $d(B, \mathfrak{s}) \leq(m+2) / 4$.

Proof of Lemma 4.3. Let $A^{\prime}$ be the 3-manifold obtained by $(m+6)$-surgery on the knot $T \#-D$. See Figure 7. Let $Y$ be the 3-manifold given by the last surgery diagram in Figure 7 . The four diagrams in Figure 7 describe a cobordism from $A \# A^{\prime}$ to $Y$. More precisely, start by taking $\left(A \# A^{\prime}\right) \times I$. Attach a 2-handle to obtain a cobordism, say, $V$, from $A \# A^{\prime}$ to the second surgery diagram in Figure 7 . Apply handle slide, to change the second surgery diagram to the third. Observe that in the third surgery diagram, the two components with framing $m+6$ and 0 give a $S^{3}$ summand, whereas the two components with framing $m$ and $2 m+7$ form a link concordant to the link in the last surgery diagram, which describes $Y$. It follows that there is a homology cobordism, say, $V^{\prime}$, from the third surgery diagram to $Y$. Now $V \cup V^{\prime}$ is a cobordism from $A \# A^{\prime}$ to $Y$.

We claim that $V$ is negative definite. To see this, view the surgery diagram of the 3-manifold $A \# A^{\prime}$ as a Kirby diagram of a 4-manifold $V_{0}$. That is, $V_{0}$ consists of one 0 -handle and three 2-handles attached 
along the framed link diagram of $A \# A^{\prime}$ in Figure 7. We have $\partial V_{0}=A \# A^{\prime}$. The second diagram in Figure 7 or, equivalently, the third diagram, is a Kirby diagram of the 4-manifold $V_{0} \cup_{A \# A^{\prime}} V$. The Kirby diagram of $V_{0}$ has linking matrix

$$
L=\left[\begin{array}{ccc}
m & -2 m-1 & 0 \\
-2 m-1 & m & 0 \\
0 & 0 & m+6
\end{array}\right]
$$

which has signature 1 because $m>0$ and the top upper $2 \times 2$ submatrix has negative determinant. It follows that $\operatorname{sign} V_{0}=1$. The third diagram in Figure 7 has linking matrix

$$
\left[\begin{array}{cccc}
m & -2 m-1 & 0 & 0 \\
-2 m-1 & 2 m+7 & 0 & 0 \\
0 & 0 & 0 & -1 \\
0 & 0 & -1 & m+6
\end{array}\right]
$$

which has vanishing signature. So sign $V_{0} \cup V=0$. By Novikov additivity, it follows that $\operatorname{sign} V=-1$. Because $b_{2}(V)=1$, this proves the claim that $V$ is negative definite.

Because $V^{\prime}$ is a homology cobordism, $H_{*}\left(V \cup V^{\prime}\right)=H_{*}(V)$. Consequently, $V \cup V^{\prime}$ is negative definite.

We will construct a generator of $H_{2}\left(V \cup V^{\prime}\right)=H_{2}(V)$ and use it to describe a certain $\operatorname{spin}^{c}$ structure on $V \cup V^{\prime}$. Let $\sigma$ be the core of the 2-handle of $\left(V, A \# A^{\prime}\right)$, and let $\alpha=\partial \sigma$ be its attaching circle, which lies in $A \# A^{\prime}$. See the second diagram in Figure 7, in which $\alpha$ is the zero-framed circle. Because the linking matrix $L$ is a presentation for $H_{1}\left(A \# A^{\prime}\right)$, it is seen that $H_{1}\left(A \# A^{\prime}\right)=\mathbb{Z}_{3 m^{2}+3 m+1} \oplus \mathbb{Z}_{m+6}$, and $\alpha=(1,1)$ in $H_{1}\left(A \# A^{\prime}\right)$. So, the order of $\alpha$ in $H_{1}\left(A \# A^{\prime}\right)$ is $\left(3 m^{2}+3 m+1\right)(m+6) / d$, where $d:=\operatorname{gcd}\left(3 m^{2}+3 m+1, m+6\right)$. (Indeed, it can be seen that $d$ is either 91 or 1.) From this, it follows that there is a 2 -cycle $z$ in $A \# A^{\prime}$ such that

$$
\partial z=\frac{\left(3 m^{2}+3 m+1\right)(m+6)}{d} \cdot \alpha
$$

Moreover, the 2-chain

$$
E:=\frac{\left(3 m^{2}+3 m+1\right)(m+6)}{d} \cdot \sigma-z
$$

is a generator of $H_{2}(V)=\mathbb{Z}$, by a standard Mayer-Vietoris argument for the 2-handle attachment.

The self-intersection number $E \cdot E$ is equal to the intersection number (in $A \# A^{\prime}$ ) of $z$ and a pushoff of $\partial z$, say, $\partial z^{\prime}$, taken along the 2-handle attachment framing (which is the zero framing in Figure 7). So $E \cdot E$ is equal to the linking number of $\partial z$ and its pushoff in the rational homology sphere $A \# A^{\prime}$. In addition, the linking number can be computed using the linking matrix $L$ (for instance, see [7, Theorem 3.1]):

$$
\begin{aligned}
E \cdot E & =\mathrm{lk}_{A \# A^{\prime}}\left(\partial z, \partial z^{\prime}\right)=\frac{\left(3 m^{2}+3 m+1\right)^{2}(m+6)^{2}}{d^{2}} \cdot\left[\begin{array}{lll}
0 & -1 & 1
\end{array}\right] L^{-1}\left[\begin{array}{c}
0 \\
-1 \\
1
\end{array}\right] \\
& =\frac{\left(3 m^{2}+3 m+1\right)(m+6)\left(-2 m^{2}+3 m-1\right)}{d^{2}} .
\end{aligned}
$$

Because the factor $-2 m^{2}+3 m-1$ of the numerator is even and $d$ is odd, $E \cdot E$ is even. From this, it follows that $0 \in H^{2}\left(V \cup V^{\prime}\right)$ is characteristic. Therefore, there is a $\operatorname{spin}^{c}$ structure $t$ on $V \cup V^{\prime}$ such that 
$c_{1}(\mathrm{t})=0$. By the Ozsváth-Szabó inequality [40, Theorem 9.6], we have

$$
d\left(Y,\left.\mathrm{t}\right|_{Y}\right)-d\left(A,\left.\mathrm{t}\right|_{A}\right)-d\left(A^{\prime},\left.\mathrm{t}\right|_{A^{\prime}}\right) \geq \frac{1}{4}
$$

Note that $A^{\prime}$ is the $(m+6)$-surgery on the $\operatorname{knot} T \#-D$, and $c_{1}\left(\left.\mathrm{t}\right|_{A^{\prime}}\right)=0$ because $c_{1}(\mathrm{t})=0$. By techniques of [11, p. 2150-2151] and [24, Appendix A], $d\left(A^{\prime},\left.\mathfrak{t}\right|_{A^{\prime}}\right)=d\left(L_{m+6}, \mathfrak{s}_{L_{m+6}}\right)$. By Ozsváth-Szabó's formula for lens spaces [40, Proposition 4.8], we have $d\left(L_{m+6}, \mathfrak{s}_{L_{m+6}}\right)=(m+5) / 4$. By Lemma 4.8, which we will prove below, we have $d\left(Y,\left.\mathrm{t}\right|_{Y}\right) \leq(2 m-1) / 4$. (Note that $c_{1}\left(\left.\mathrm{t}\right|_{Y}\right)=0$ because $c_{1}(\mathrm{t})=0$.) Combining these with the above inequality, it follows that $d(A, \mathfrak{s}) \leq(m-7) / 4$.

\subsection{A quick summary of Némethi's method for Seifert 3-manifolds}

For the reader's convenience, we provide a summary of Némethi's method to compute $d$-invariants [38], which we will use in Subsection 4.3. We focus on the case of Seifert 3-manifolds, which is treated in [38, Section 11], and [38] provides techniques for a larger class of certain plumbed 3-manifolds.

Let $Y$ be a Seifert 3-manifold. It is well known that $Y$ admits a surgery presentation of a specific form, which is shown in the left side of Figure 8. The associated star-shaped plumbing graph, which is shown in the right side of Figure 8, is often used to describe the 3-manifold $Y$, as the boundary of a plumbed 4-manifold $X$ : for each vertex, take a disk bundle over a 2-sphere whose Euler number is the integer decoration of the vertex. For each edge, perform +1 plumbing between the two disk bundles corresponding to the endpoints. The result is a 4-manifold $X$ with $\partial X=Y$.

Let $v$ be the number of branches of the star-shaped graph in Figure 8. In this subsection, we assume that $v \geq 3$ and that $X$ is negative definite. (We remark that not all Seifert 3-manifolds $Y$ are described by a plumbing graph satisfying this assumption.)

We use the following notation. Let $e_{0}$ be the decoration of the root vertex. Let $s_{\ell}$ be the number of (nonroot) vertices on the $\ell$ th branch, and let $e_{\ell, 1}, \ldots, e_{\ell, s_{\ell}}$ be the decorations of those $s_{\ell}$ vertices. See Figure 8 . We may assume that $e_{\ell, j} \leq-2$ for all $\ell, j$ (for instance, see [39]). Let $b_{0}$ and $b_{\ell, j} \in H_{2}(X)$ be the classes of 2 -spheres corresponding to vertices with decoration $e_{0}$ and $e_{\ell, j}$ respectively $\left(1 \leq \ell \leq v, 1 \leq j \leq s_{\ell}\right)$. They form a basis for the free abelian group $H_{2}(X)$. Let $b_{0}^{*}$ and $b_{\ell, j}^{*} \in H^{2}(X)$ be basis elements (hom) dual to $b_{0}$ and $b_{\ell, j}$. With respect to these bases, the intersection form $\lambda: H_{2}(X) \times H_{2}(X) \rightarrow X$ or its adjoint $\lambda: H_{2}(X) \rightarrow \operatorname{Hom}\left(H_{2}(X), \mathbb{Z}\right)=H^{2}(X)$ is given as follows: $\lambda\left(b_{0}, b_{0}\right)=e_{0}, \lambda\left(b_{\ell, j}, b_{\ell, j}\right)=e_{\ell, j}$, and for $b \neq b^{\prime}, \lambda\left(b, b^{\prime}\right)=1$ if $\left\{b, b^{\prime}\right\}=\left\{b_{0}, b_{\ell, 1}\right\}$ or $\left\{b_{\ell, j}, b_{\ell, j+1}\right\}$, and $\lambda\left(b, b^{\prime}\right)=0$ otherwise.
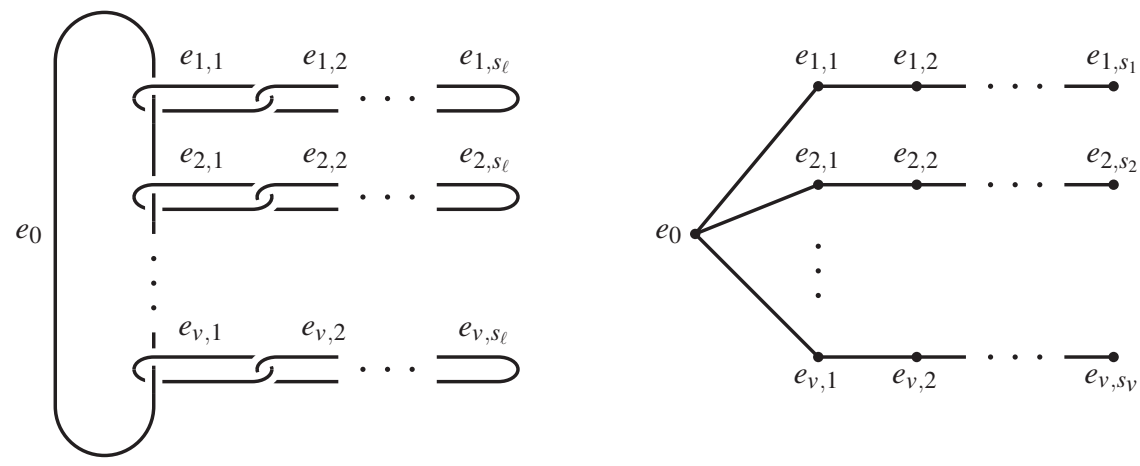

Figure 8. A Seifert 3-manifold and its plumbing graph. 
For $1 \leq \ell \leq v$, define a continued fraction by

$$
\frac{\alpha_{\ell}}{\omega_{\ell}}:=\left[-e_{\ell, 1}, \ldots,-e_{\ell, s_{\ell}}\right]=-e_{\ell, 1}-\frac{1}{-e_{\ell, 2}-\frac{1}{\vdots}} .
$$

The condition $e_{\ell, j} \leq-2$ implies that $\alpha_{\ell} / \omega_{\ell}>1$. So we may assume $0 \leq \omega_{\ell}<\alpha_{\ell}$. Then, a standard diagonalisation process applied to the intersection form $\lambda$ gives us a diagonal matrix whose all diagonals are easily seen to be negative, possibly except one, which is equal to

$$
e:=e_{0}+\sum_{\ell=1}^{v} \omega_{\ell} / \alpha_{\ell} .
$$

So, $X$ is negative definite if and only if $e<0$.

The set of characteristic elements in $H^{2}(X)$ is defined to be

$$
\operatorname{Char}(X):=\left\{\xi \in H^{2}(X) \mid \xi\left(b_{j}\right) \equiv \lambda\left(b_{j}, b_{j}\right) \bmod 2 \text { for all } j\right\} .
$$

The Chern class $c_{1}: \operatorname{Spin}^{c}(X) \rightarrow \operatorname{Char}(X)$ is bijective, because $H^{2}(X)$ does not have any 2-torsion. Under the identification via $c_{1}$, the action of an element $c \in H^{2}(X)$ on $\operatorname{Spin}^{c}(X)$ is given by $\xi \mapsto \xi+2 c$ for $\xi \in \operatorname{Char}(X)=\operatorname{Spin}^{c}(X)$. In particular, the action of $x \in H_{2}(X)$ on $\operatorname{Char}(X)=\operatorname{Spin}^{c}(X)$ via $\lambda: H_{2}(X) \rightarrow H^{2}(X)$ is given by $\xi \mapsto \xi+2 \lambda(x)$.

The Chern class $c_{1}: \operatorname{Spin}^{c}(Y) \rightarrow H^{2}(Y)$ is not injective in general, so the standard identification of $\operatorname{spin}^{c}$ structures of $Y$ is given indirectly using $X$ : we have a bijection

$$
\operatorname{Spin}^{c}(Y) \approx \operatorname{Char}(X) / 2 \lambda\left(H_{2}(X)\right) .
$$

Here, for a $\operatorname{spin}^{c}$ structure $\xi \in \operatorname{Spin}^{c}(X)=\operatorname{Char}(X)$, the coset $[\xi]=\xi+2 \lambda\left(H_{2}(X)\right)$ in $\operatorname{Char}(X) / 2 \lambda\left(H_{2}(X)\right)$ corresponds to the restriction of $\xi$ on $Y$. Essentially, the bijectivity is a consequence of the fact that $H^{2}(Y)$ is the cokernel of $\lambda: H_{2}(X) \rightarrow H^{2}(X)$.

In [38], the notion of a distinguished representative is used to express a $\operatorname{spin}^{c}$ structure of $Y$. Instead of the original definition (see Section 5, especially Definition 5.1 of [38]), we will use a characterisation theorem as a definition. We need the following notation. For $1 \leq i \leq j \leq s_{\ell}$, let $n_{i, j}^{\ell} / d_{i, j}^{\ell}:=\left[-e_{i}^{\ell}, \ldots,-e_{j}^{\ell}\right]$, where $n_{i, j}^{\ell}>0$ and $\operatorname{gcd}\left(n_{i, j}^{\ell}, d_{i, j}^{\ell}\right)=1$. Note that $\alpha_{\ell} / \omega_{\ell}=n_{1, s_{\ell}}^{\ell} / d_{1, s_{\ell}}^{\ell}$. Define an element $K \in \operatorname{Spin}^{c}(X)=\operatorname{Char}(X) \subset H^{2}(X)$ by

$$
K\left(b_{0}\right)=-e_{0}-2, \quad K\left(b_{\ell, j}\right)=-e_{\ell, j}-2 \text { for } 1 \leq \ell \leq v, 1 \leq j \leq s_{\ell} .
$$

The element $K$ is characteristic because $K\left(b_{0}\right) \equiv \lambda\left(b_{0}, b_{0}\right)$ and $K\left(b_{\ell, j}\right) \equiv \lambda\left(b_{\ell, j}, b_{\ell, j}\right) \bmod 2$. In [38], $K$ is called the canonical $\operatorname{spin}^{c}$ structure. We have $\operatorname{Char}(X)=K+2 H^{2}(X)$. Consider a class $k_{r} \in \operatorname{Char}(X)$ of the form

$$
k_{r}=K-2\left(a_{0} b_{0}^{*}+\sum_{\ell, j} a_{\ell, j} b_{\ell, j}^{*}\right)
$$

where $a_{0}$ and $a_{\ell, j}$ are integers. Let

$$
a_{\ell}=\sum_{t=1}^{s_{\ell}-1} n_{t+1, s_{\ell}}^{\ell} a_{\ell, t} .
$$


Definition 4.5 ([38, Corollary 11.7]). The class $k_{r}$ in (4.3) called a distinguished representative if $0 \leq a_{\ell}<\alpha_{\ell}$ for all $\ell$ and if

$$
0 \leq a_{0} \leq-1-i e_{0}-\sum_{\ell=1}^{v}\left[\frac{i \omega_{\ell}+a_{\ell}}{\alpha_{\ell}}\right]
$$

for all $i>0$. Here $[x]$ is the largest integer not greater than $x$.

To state Némethi's formula for the $d$-invariant, we need one more notation. Let

$$
\tau(i)=\sum_{t=0}^{i-1}\left(a_{0}+1-t e_{0}+\sum_{\ell=1}^{v}\left[\frac{-t \omega_{\ell}+a_{\ell}}{\alpha_{\ell}}\right]\right) .
$$

In particular, $\tau(0)=0$.

Theorem 4.5 (Némethi [38, p. 1038]). Suppose that $X$ is negative definite and $v \geq 3$. Suppose that the class $k_{r}$ given in (4.3) is a distinguished representative. Then, for the $\operatorname{spin}^{c}$ structure $\left[k_{r}\right]$ of $Y$, the d-invariant is given by

$$
d\left(Y,\left[k_{r}\right]\right)=\frac{k_{r}^{2}+b_{2}(X)}{4}-2 \cdot \min \{\tau(i) \mid i \geq 0\}
$$

\section{Remark 4.6.}

(1) For each $k_{r}$ given by (4.3), the minimum in Theorem 4.6 can be found in finite steps. To see this, let $\Delta_{i}=\tau(i+1)-\tau(i)$. Then we have

$$
\begin{aligned}
\Delta_{i} & \geq a_{0}+1-i e_{0}+\sum_{\ell=1}^{v} \frac{-i \omega_{\ell}+a_{\ell}+\alpha_{\ell}-1}{\alpha_{\ell}} \\
& =-e \cdot i+\left(1+a_{0}+\sum_{\ell=1}^{v} \frac{a_{\ell}-\alpha_{\ell}+1}{\alpha_{\ell}}\right) \geq 0
\end{aligned}
$$

if $i$ is not smaller than

$$
R:=\left(1+a_{0}+\sum_{\ell=1}^{v} \frac{a_{\ell}-\alpha_{\ell}+1}{\alpha_{\ell}}\right) /(-e) .
$$

Here we use that $e$ is negative because $X$ is negative definite. So, the minimum in Theorem 4.6 can be taken over $0 \leq i \leq \max \{0, R\}$.

(2) A similar argument shows that it can be determined in finite steps whether a class $k_{r}$ given by (4.3) satisfies Definition 4.5. Indeed, the right- hand side of (4.5) is bounded from below by $-\left(1+\sum a_{\ell} / \alpha_{\ell}\right)-e i$. Because $e<0,(4.5)$ is satisfied for all large $i$, and thus it suffices to check (4.5) for only finitely many $i$.

Using (1) and (2), it is straightforward to write a practically efficient algorithm (and computer code) to find distinguished representatives of all $\operatorname{spin}^{c}$ structures of $Y$ and compute the associated $d$-invariants.

\section{3. d-invariants of the 3-manifolds $Y$ and $B$}

Let $Y$ be the 3-manifold given by the last surgery diagram in Figure 7 or, equivalently, by the first surgery diagram in Figure 9. The following lemma gives an estimate of the $d$-invariant of $Y$, which is used to complete the proof of Lemma 4.3. Note that there are two $\operatorname{spin}^{c}$ structures on $Y$ satisfying $c_{1}(\mathfrak{s})=0$, because $\left|H_{1}(Y)\right|=2 m^{2}-3 m+1$ is even. 

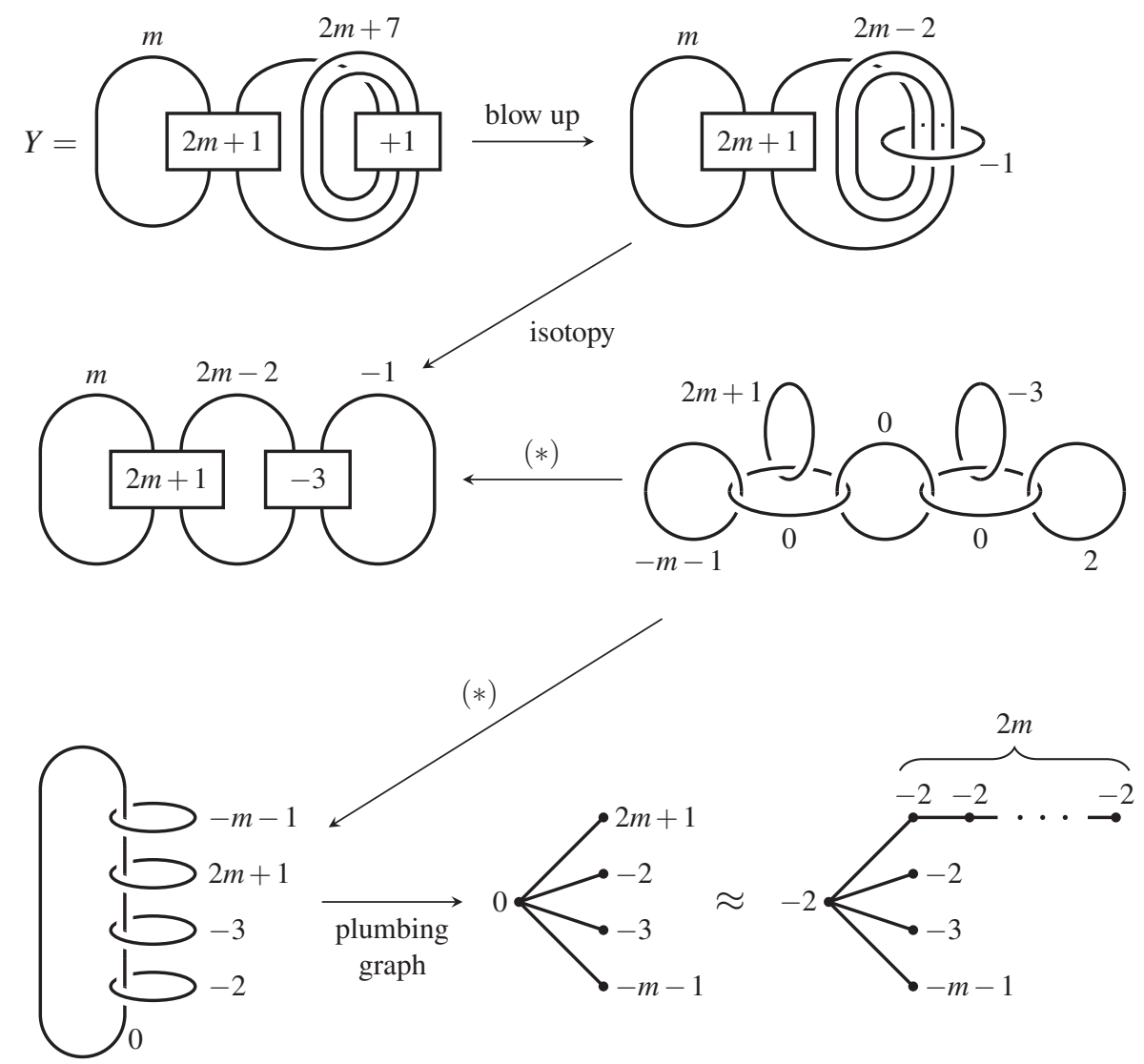

Figure 9. Surgery diagram calculus that gives a plumbing tree for $Y$. The symbol (*) means handle slides and elimination of components with 0 -framed meridians.

Lemma 4.8. If $\mathfrak{s}$ is a spinc ${ }^{c}$ structure on $Y$ such that $c_{1}(\mathfrak{s})=0$, then $d(Y, \mathfrak{s}) \leq(2 m-1) / 4$.

Proof. The surgery diagram calculus in Figure 9 shows that $Y$ is a Seifert 3-manifold. The last plumbing graph in Figure 9 describes a plumbed 4-manifold $X$ with $\partial X=Y$.

To compute the $d$-invariant, we will apply the method discussed in Subsection 4.2. Using the notation in Subsection 4.2, denote the basis of $H_{2}(X)$ by $\left\{b_{0}, b_{1,1}, \ldots, b_{1,2 m}, b_{2,1}, b_{3,1}, b_{4,1}\right\}$ and the dual basis of $H^{2}(X)$ by $\left\{b_{0}^{*}, b_{1,1}^{*}, \ldots, b_{1,2 m}^{*}, b_{2,1}^{*}, b_{3,1}^{*}, b_{4,1}^{*}\right\}$. The intersection form $\lambda: H_{2}(X) \times H_{2}(X) \rightarrow \mathbb{Z}$ is computed straightforwardly from the plumbing graph:

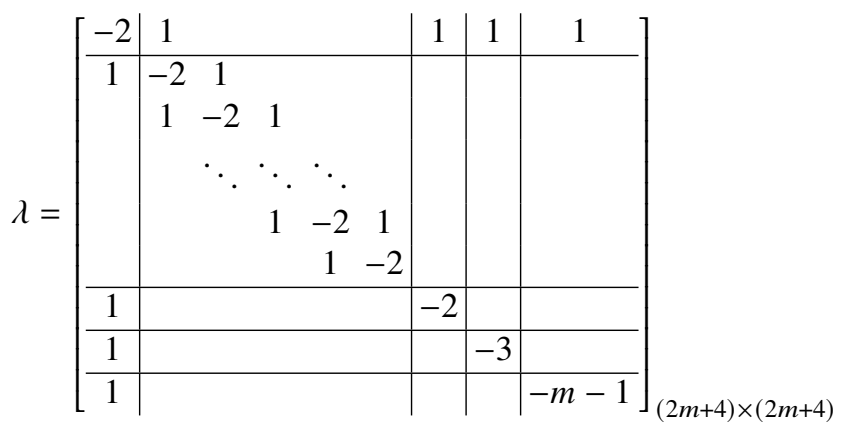


Also, using the definition in Subsection 4.2, it is routine to compute the following:

$$
\left(\frac{\alpha_{1}}{\omega_{1}}, \frac{\alpha_{2}}{\omega_{2}}, \frac{\alpha_{3}}{\omega_{3}}, \frac{\alpha_{4}}{\omega_{4}}\right)=\left(\frac{2 m+1}{2 m}, \frac{2}{1}, \frac{3}{1}, \frac{m+1}{1}\right) .
$$

So, the orbifold Euler number is given by

$$
e=-2+\frac{2 m}{2 m+1}+\frac{1}{2}+\frac{1}{3}+\frac{1}{m+1}=-\frac{(m-1)(2 m-1)}{6(m+1)(2 m+1)} .
$$

For $m>1$, we have $e<0$, so $X$ is negative definite.

We will describe two $\operatorname{spin}^{c}$ structures $\left[k_{1}\right]$ and $\left[k_{2}\right] \in \operatorname{Char}(X) / 2 \lambda\left(H_{2}(X)\right)$. Let

$$
\begin{aligned}
& k_{1}:=-2 b_{1,2 m}^{*}-b_{3,1}^{*}+2 b_{4,1}^{*}, \\
& k_{2}:=-2 b_{1,2 m-1}^{*}+b_{3,1}^{*}-2 b_{4,1}^{*} .
\end{aligned}
$$

It is straightforward to show that $k_{1}$ and $k_{2}$ are distinguished representatives in the sense of Definition 4.5. Indeed, in our case, the canonical $\operatorname{spin}^{c}$ structure described in (4.2) is given by $K=b_{3,1}^{*}-(m+1) b_{4,1}^{*}$, and $k_{1}$ is of the form (4.3), where

$$
a_{0}=0, \quad\left(a_{1,1}, \ldots, a_{1,2 m}\right)=(0, \ldots, 0,1), \quad a_{2,1}=0, \quad a_{3,1}=1, \quad a_{4,1}=1 .
$$

By (4.4), we have $\left(a_{0}, a_{1}, a_{2}, a_{3}, a_{4}\right)=\left(0,1,0,1, \frac{m-3}{2}\right)$. This satisfies the conditions in Definition 4.5, so $k_{1}$ is a distinguished representative. The class $k_{2}$ is shown to be a distinguished representative, too, by similar computation. In this case, we have

$$
a_{0}=0, \quad\left(a_{1,1}, \ldots, a_{1,2 m}\right)=(0, \ldots, 0,1,0), \quad a_{2,1}=0, \quad a_{3,1}=0, \quad a_{4,1}=m-2
$$

and $\left(a_{0}, a_{1}, a_{2}, a_{3}, a_{4}\right)=(0,2,0,0, m-2)$.

Under the adjoint $\lambda: H_{2}(X) \rightarrow H^{2}(X)=\operatorname{Hom}\left(H_{2}(X), \mathbb{Z}\right)$ of the intersection form of $X, k_{1}$ and $k_{2}$ are respectively the images of

$$
\begin{aligned}
& x_{1}=2 b_{0}+2 b_{1,1}+\cdots+2 b_{1,2 m}+b_{2,1}+b_{3,1}, \\
& x_{2}=4 b_{1}+4 b_{1,1}+\cdots+4 b_{1,2 m-1}+2 b_{1,2 m}+2 b_{2,1}+2 b_{3,1}+2 b_{4,1} .
\end{aligned}
$$

Recall that $\operatorname{Char}(X) \subset H^{2}(X)$ is identified with $\operatorname{Spin}^{c}(X)$ via $c_{1}$, and thus for a $\operatorname{spin}^{c}$ structure $[k] \in \operatorname{Char}(X) / 2 \lambda\left(H_{2}(X)\right)$ of $Y$, we have $c_{1}([k])=\left.k\right|_{Y}$. So, $c_{1}([k])=0$ if and only if $k$ lies in the kernel of $H^{2}(X) \rightarrow H^{2}(Y)$ or, equivalently, $k$ lies in the image of $\lambda: H_{2}(X) \rightarrow H^{2}(X)$. From this observation, it follows that $c_{1}\left(\left[k_{i}\right]\right)=0$ for $i=1,2$, because $k_{i}=\lambda\left(x_{i}\right)$. Also, $\left[k_{1}\right] \neq\left[k_{2}\right]$ because $x_{1}-x_{2} \notin 2 H_{2}(X)$. Therefore, to complete the proof, it suffices to show $d\left(Y,\left[k_{i}\right]\right) \leq(2 m-1) / 4$ for $i=1,2$.

We have

$$
k_{1}^{2}=\lambda\left(x_{1}, x_{1}\right)=-3, \quad k_{2}^{2}=\lambda\left(x_{2}, x_{2}\right)=-m-4 .
$$

The last thing we need is the minimum of the values of $\tau(i)$ defined in (4.6). Recall the notation $\Delta_{i}=\tau(i+1)-\tau(i)$ from Remark 4.7.

Assertion. For both $k_{1}$ and $k_{2}$ and for all $i \geq 0, \Delta_{i} \geq 0$.

We will provide a proof of the assertion for $m \geq 23$. For $m<23$, the assertion is verified by direct inspection using Remark 4.7 (indeed, the author used a computer program), so we omit details for $m<23$. Note that it suffices to use $m \geq 23$ to prove the main results of this article, Theorems A, B, C and D. 
For the case of $k_{1}$, by Remark 4.7, we have $\Delta_{i} \geq 0$ for $i \geq R$, where

$$
R=8+(48 m-6) /(2 m-1)(m-1)
$$

Because $m \geq 23, R \leq 10$. So, $\Delta_{i} \geq 0$ for $i \geq 10$. For $i \leq 10$, using (4.6), we have

$$
\begin{aligned}
\Delta_{i} & =1+2 i+\left[\frac{-2 m i+1}{2 m+1}\right]+\left[\frac{-i}{2}\right]+\left[\frac{-i+1}{3}\right]+\left[\frac{-i+(m-3) / 2}{m+1}\right] \\
& \geq 1+2 i-i+\frac{-i-1}{2}+\frac{-i-1}{3}=\frac{i+1}{6} \geq 0 .
\end{aligned}
$$

This shows the claim for $k_{1}$. For the case of $k_{2}$, we proceed in the same way. We have

$$
R=7+(48 m-6) /(2 m-1)(m-1) .
$$

Because $m \geq 23, R \geq 10$, and thus $\Delta_{i} \geq 0$ for $i \geq 10$ by Remark 4.7. For $1 \leq i \leq 10$, using (4.6), we have

$$
\begin{aligned}
\Delta_{i} & =1+2 i+\left[\frac{-2 m i+1}{2 m+1}\right]+\left[\frac{-i}{2}\right]+\left[\frac{-i}{3}\right]+\left[\frac{-i+m-2}{m+1}\right] \\
& \geq 1+2 i-i+\frac{-i-1}{2}+\frac{-i-2}{3}=\frac{i-1}{6} \geq 0 .
\end{aligned}
$$

For $i=0$, a direct computation using (4.6) gives $\Delta_{0}=1$. This completes the proof of the assertion.

From the assertion, it follows that $\min \{\tau(i) \mid i \geq 0\}=0$ because $\tau(0)=0$. So, by Némethi's Theorem 4.6,

$$
d\left(Y,\left[k_{i}\right]\right)=\frac{k_{i}^{2}+2 m+4}{4}= \begin{cases}(2 m-1) / 4 & \text { for } i=1, \\ m / 4 & \text { for } i=2 .\end{cases}
$$

Therefore $d\left(Y,\left[k_{i}\right]\right) \leq(2 m-1) / 4$ holds for $i=1,2$.

Now, to complete the proof of Theorem 2.2, it only remains to prove of Lemma 4.4, which estimates $d$-invariants of the 3-manifold $B$ in Figure 6. In the proof below, we will use that $B$ is a Seifert 3-manifold as well.

Proof of Lemma 4.4. Recall that $B$ is the 3-manifold described in Figure 6. Let $\mathfrak{s}$ be a $\operatorname{spin}^{c}$ structure of $B$ satisfying $c_{1}(\mathfrak{s})=0$. The goal is to show that $d(B, \mathfrak{s}) \leq(m+2) / 4$.

As we will see in what follows, it will be useful to consider $-B$ instead of $B$. That is, we will show $d(-B, \mathfrak{s}) \geq-(m+2) / 4$. Figure 10 shows that $-B$ is a Seifert 3-manifold. We use the notation of Subsection 4.2. The associated plumbed 4-manifold $X$ has $H_{2}(X)=\mathbb{Z}^{2 m}$, with basis elements $b_{0}$, $b_{1,1}, \ldots, b_{1, m}, b_{2,1}, \ldots, b_{2, m-2}, b_{3,1}$. The associated decorations are $e_{0}=-2, e_{1, j}=-2$ for all $j$, $e_{2, j}=-2$ for all $j$ and $e_{3,1}=-2 m-1$. Let $b_{0}^{*}, b_{\ell, j}^{*}$ be the dual basis elements in $H^{2}(X)$. Because

$$
e=-2+\frac{m}{m+1}+\frac{m-2}{m-1}+\frac{1}{2 m+1}=\frac{-3 m^{2}-2 m-1}{\left(m^{2}-1\right)(2 m+1)}<0,
$$

the 4-manifold $X$ is negative definite. This is why we use $-B$ instead of $B$.

The canonical $\operatorname{spin}^{c}$ structure $K$ of $X$ is given by $K=(2 m-1) b_{3,1}^{*}$. Let

$$
\begin{aligned}
& x_{1}=b_{1,1}+b_{1,3}+\cdots+b_{1, m}+b_{3,1}, \\
& x_{2}=b_{2,1}+b_{2,3}+\cdots+b_{2, m-2}+b_{3,1},
\end{aligned}
$$

and let $k_{i}=\lambda\left(x_{i}\right) \in H^{2}(X)$ for $i=1$, 2. Then $k_{i} \in \operatorname{Char}(X)=K+2 H^{2}(X)$, so that $\left[k_{i}\right] \in$ $\operatorname{Char}(X) / 2 \lambda\left(H_{2}(X)\right)=\operatorname{Spin}^{c}(-B)$ is a $\operatorname{spin}^{c}$ structure of $-B$. Similar to the proof of Lemma 4.8, 


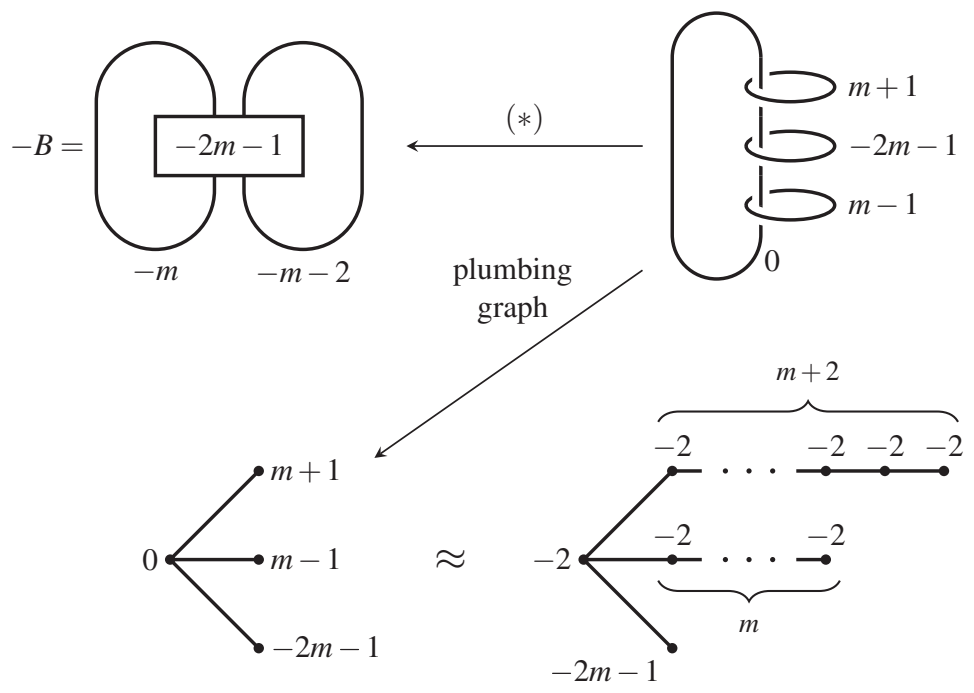

Figure 10. Surgery diagram calculus showing that $-B$ is a Seifert 3-manifold. The symbol (*) means handle slides (or Rolfsen twist [41, p. 264]) followed by elimination of a component together with a 0 -framed meridian.

$c_{1}\left(\left[k_{i}\right]\right)=\left.k_{i}\right|_{-B}=0$. Also, $\left[k_{1}\right] \neq\left[k_{2}\right]$ in $\operatorname{Spin}^{c}(-B)$ because $x_{1}-x_{2} \notin 2 H_{2}(X)$. It follows that $\left[k_{1}\right]$ and $\left[k_{2}\right]$ are the two $\operatorname{spin}^{c}$ structures of $-B$ with $c_{1}=0$. So, it suffices to show that $d\left(-B,\left[k_{i}\right]\right) \geq-(m+2) / 4$ for $i=1,2$. Instead of determining the values exactly, we will present a simpler argument that gives the promised estimate. We have $k_{1}^{2}=\lambda\left(x_{1}, x_{1}\right)=-3 m-2$. So, by Ozsváth-Szabó's inequality [40, Theorem 9.6],

$$
d\left(-B,\left[k_{1}\right]\right) \geq \frac{k_{1}^{2}+b_{2}(X)}{4}=\frac{-m-2}{4} .
$$

Similarly, because $k_{2}^{2}=\lambda\left(x_{2}, x_{2}\right)=-3 m$, we have

$$
d\left(-B,\left[k_{2}\right]\right) \geq \frac{k_{2}^{2}+b_{2}(X)}{4}=\frac{-m}{4} .
$$

So, we have $d\left(-B,\left[k_{i}\right]\right) \geq-(m+2) / 4$ for $i=1,2$. (Indeed, it can be shown that the equality holds in (4.7) and (4.8), by using the technique described in Subsection 4.2, as we did in the proof of Lemma 4.8.)

\section{Appendix. General primary decomposition}

The goal of this appendix is to present an abstract formulation of the notion of primary decomposition along an invariant with values in an unique factorisation domain. We also discuss questions in specific cases and earlier related results in the literature from our viewpoint. The organisation is as follows. In Subsection A.1, we describe the definition of general primary decomposition and present basic observations. In Subsection A.2, we investigate primary decomposition of extensions. In Subsection A.3, we discuss specialisations to various knot concordance groups (e.g., smooth/topological) and related filtrations. In Subsection A.4, we discuss the case of rational homology cobordism group of rational homology 3-spheres. 


\section{A.1. Definitions and basic observations}

Let $\mathbb{K}$ be an abelian monoid and $\sim$ be an equivalence relation on $\mathbb{K}$. Suppose that the monoid structure on $\mathbb{K}$ descends to an abelian group structure on the set $\mathcal{C}:=\mathbb{K} / \sim$ of equivalence classes. Denote the equivalence class of $K \in \mathbb{K}$ by $[K] \in \mathcal{C}$.

Let $R$ be a unique factorisation domain with involution $r \mapsto r^{*}$. Main examples are $\mathbb{Z}$ with a trivial involution and the Laurent polynomial ring $\mathbb{Q}\left[t^{ \pm 1}\right]$ with the standard involution $\left(\sum a_{i} t^{i}\right)^{*}=\sum a_{i} t^{-i}$. For $r, s \in R$, write $r \doteq s$ if $r$ and $s$ in $R$ are associates; that is, $r=u s$ for some unit $u$ in $R$. We say that two irreducibles $\lambda$ and $\mu$ in $R$ are $*$-associates if either $\lambda \doteq \mu$ or $\lambda^{*} \doteq \mu$. We say that $r \in R$ is self-dual if $r \doteq r^{*}$.

Suppose that $\chi: \mathbb{K} \rightarrow(R \backslash\{0\}) / \doteq$ is a function. We will denote a representative of $\chi(K)$ by $\Delta_{K} \in R \backslash\{0\}$. Suppose that the following hold for all $K, K^{\prime}$ in $\mathbb{K}$ :

$(\Delta 1) \Delta_{K}$ is self-dual.

$(\Delta 2) \Delta_{K+K^{\prime}} \doteq \Delta_{K} \cdot \Delta_{K^{\prime}}$.

$(\Delta 3)-[K]=[J]$ for some $J$ in $\mathbb{K}$ such that $\Delta_{J} \doteq \Delta_{K}$.

In particular, writing $\mathcal{S}(R)=\{r \in R \mid r \neq 0$ is self-dual $\}, \mathbb{K} \stackrel{\chi}{\rightarrow} \mathcal{S}(R) / \doteq$ is a homomorphism between abelian monoids.

Of course, the main example that one may keep in mind is the case that $\mathbb{K}$ is the monoid of knots under connected sum, $\sim$ is concordance and $\Delta_{K}$ is the Alexander polynomial in $R=\mathbb{Q}\left[t^{ \pm 1}\right]$. Including this, we will discuss various specific examples in Subsections A.3 and A.4.

Let $\Delta=\left\{[K] \in \mathcal{C} \mid \Delta_{K} \doteq 1\right\}$. For an irreducible $\lambda$ in $R$, let $S(\lambda)=\lambda$ if $\lambda$ is self-dual, and $S(\lambda)=\lambda \lambda^{*}$ otherwise. Let

$$
\begin{aligned}
& \mathcal{C}_{\lambda}:=\left\{[K] \in \mathcal{C} \mid \Delta_{K} \doteq S(\lambda)^{k} \text { for some } k \geq 0\right\}, \\
& \mathcal{C}^{\lambda}:=\left\{[K] \in \mathcal{C} \mid \Delta_{K} \text { is relatively prime to } \lambda\right\} .
\end{aligned}
$$

It is straightforward to verify that $\mathcal{C}_{\lambda}, \mathcal{C}^{\lambda}$ and $\Delta$ are subgroups of $\mathcal{C}$, using ( $\left.\Delta 2\right)$ and ( $\left.\Delta 3\right)$. Also, using $(\Delta 1)$, it is seen that $[K] \in \mathcal{C}_{\lambda}$ if and only if $K \sim J$ for some $J$ with $\Delta_{J}$ lying in the multiplicative subset generated by $\lambda, \lambda^{*}$ and the units in $R$. We have $\Delta \subset \mathcal{C}_{\lambda}, \mathcal{C}_{\lambda}=\mathcal{C}_{\lambda^{*}}$ and $\mathcal{C}^{\lambda}=\mathcal{C}^{\lambda^{*}}$. Use $S(\lambda)=S\left(\lambda^{*}\right)$ and $(\Delta 1)$ to verify the two equalities respectively.

Note that though $\Delta_{K}$ is self-dual, irreducible factors of $\Delta_{K}$ are not necessarily self-dual. For instance, in $R=\mathbb{Q}\left[t^{ \pm 1}\right]$, we have $-2 t+5-2 t^{-1}=(t-2)\left(t^{-1}-2\right)$. By the above definition, a class $[K]$ with $\Delta_{K}=-2 t+5-2 t^{-1}$ lies in $\mathcal{C}_{\lambda}$ for $\lambda=t-2$.

Definition A.1. Let $\mathbb{P}$ be the set of $*$-associate classes of irreducibles in $R$.

(1) We say that $(\mathbb{K}, \sim, \chi)$ is left primary decomposable if the sum

$$
\Phi_{L}: \bigoplus_{[\lambda] \in \mathbb{P}} \mathcal{C}_{\lambda} / \Delta \longrightarrow \mathcal{C} / \Delta
$$

of the inclusions $\mathcal{C}_{\lambda} / \Delta \hookrightarrow \mathcal{C} / \Delta$ is an isomorphism.

(2) We say that $(\mathbb{K}, \sim, \chi)$ is right primary decomposable if the surjections $\mathcal{C} / \Delta \rightarrow \mathcal{C} / \mathcal{C}^{\lambda}$ induce an isomorphism

$$
\Phi_{R}: \mathcal{C} / \Delta \longrightarrow \bigoplus_{[\lambda] \in \mathbb{P}} \mathcal{C} / \mathcal{C}^{\lambda}
$$

Because each $\Delta_{K}$ has finitely many irreducible factors, it follows that the product $\mathcal{C} / \Delta \rightarrow$ $\prod_{[\lambda] \in \mathbb{P}} \mathcal{C} / \mathcal{C}^{\lambda}$ of the surjections $\mathcal{C} / \Delta \rightarrow \mathcal{C} / \mathcal{C}^{\lambda}$ has image in the direct sum $\bigoplus_{[\lambda] \in \mathbb{P}} \mathcal{C} / \mathcal{C}^{\lambda}$. That is, $\Phi_{R}$ is always a well-defined homomorphism. 
We remark that taking the quotient by $\Delta$ may be viewed as an analogue of ignoring units in the primary decomposition in a unique factorisation domain.

For brevity, when the choice of $(\mathbb{K}, \sim, \chi)$ is clearly understood from the context, we will simply say that $\mathcal{C}$ is left or right primary decomposable.

From the definition, it is straightforward to see that $\mathcal{C}$ is left primary decomposable if only if the following two conditions (LP1) and (LP2) hold. More precesly, (LP1) and (LP2) are respectively equivalent to the surjectivity and injectivity of $\Phi_{L}$.

(LP1) Existence: for every $K \in \mathbb{K}$, there exist irreducibles $\lambda_{1}, \ldots, \lambda_{n}$ and $\left[K_{1}\right] \in \mathcal{C}_{\lambda_{1}}, \ldots,\left[K_{n}\right] \in \mathcal{C}_{\lambda_{n}}$ such that $[K] \equiv\left[K_{1}\right]+\cdots+\left[K_{n}\right] \bmod \Delta$.

(LP2) Uniqueness: if $\left[K_{1}\right]+\cdots+\left[K_{n}\right] \equiv 0 \bmod \Delta$ and $\left[K_{i}\right] \in \mathcal{C}_{\lambda_{i}}$ for some pairwise distinct $\left[\lambda_{1}\right], \ldots$, $\left[\lambda_{n}\right] \in \mathbb{P}$, then $\left[K_{i}\right] \equiv 0 \bmod \Delta$ for all $i$.

The following examples illustrate that the left and right primary decomposabilities are independent of each other.

Example A.2. Let

$$
\mathbb{K}=\left\{a K+b K^{\prime}+c J+d J^{\prime} \mid a, b, c, d \geq 0\right\} \cong\left(\mathbb{Z}_{\geq 0}\right)^{4}
$$

be the free abelian monoid generated by four generators $K, K^{\prime}, J$ and $J^{\prime}$. Define $\sim$ on $\mathbb{K}$ by

$$
a K+b K^{\prime}+c J+d J^{\prime} \sim p K+q K^{\prime}+r J+s J^{\prime} \Longleftrightarrow a-b+c-d=p-q+r-s
$$

and let $\mathcal{C}=\mathbb{K} / \sim$. Then $\mathcal{C}$ is the infinite cyclic group, and $[K]=-\left[K^{\prime}\right]=[J]=-\left[J^{\prime}\right]$ is a generator. Fix $R$ that has three distinct self-dual irreducibles $\lambda, \mu$ and $\nu$. Define

$$
\Delta_{a K+b K^{\prime}+c J+d J^{\prime}}=\lambda^{a+b}(\mu v)^{c+d} .
$$

In particular, $\Delta_{K}=\lambda$ and $\Delta_{J}=\mu \nu$. It is straightforward to verify that $(\Delta 1),(\Delta 2)$ and $(\Delta 3)$ are satisfied and that the subgroup $\Delta$ is trivial.

We claim that $\mathcal{C}$ is left primary decomposable. Indeed, it is straightforward to see that for $L \in \mathbb{K}$, $\Delta_{L}$ is a power of an irreducible if and only if $L=a K+b K^{\prime}$ (and thus $\Delta_{L}=\lambda^{a+b}$ ). It follows that for any irreducible $\zeta$ in $R, \mathcal{C}_{\zeta}=\mathcal{C}$ if $\zeta=\lambda$, and $\mathcal{C}_{\zeta}=0$ otherwise. So $\Phi_{L}: \bigoplus_{[\zeta]} \mathcal{C}_{\zeta} / \Delta \rightarrow \mathcal{C} / \Delta$ is an isomorphism.

On the other hand, $\mathcal{C}$ is not right primary decomposable. To see this, observe that $[K] \in \mathcal{C}^{\zeta}$ for all $\zeta \neq \lambda$, because $\Delta_{K}=\lambda$. Also, $[K]=[J] \in \mathcal{C}^{\lambda}$ because $\Delta_{J}=\mu \nu$. Because $[K]$ generates $\mathcal{C}$, it follows that $\mathcal{C}^{\zeta}=\mathcal{C}$ for all $\zeta$. So $\Phi_{R}: \mathcal{C} / \Delta \rightarrow \bigoplus_{[\zeta]} \mathcal{C} / \mathcal{C}^{\zeta}=0$ is not injective.

Example A.3. Let $\mathbb{K}, \sim, \mathcal{C}, R, \lambda, \mu$ and $v$ be as in Example A.2, but define

$$
\Delta_{a K+b K^{\prime}+c J+d J^{\prime}}=(\lambda \mu)^{a+b}(\mu \nu)^{c+d} .
$$

Note that $(\Delta 1),(\Delta 2)$ and $(\Delta 3)$ are satisfied, $\Delta_{K}=\lambda \mu, \Delta_{J}=\mu v$ and $\Delta$ is trivial.

First we will show that $\mathcal{C}$ is not left primary decomposable. For $L \in \mathbb{K}$, either $L=0$ or $\Delta_{L}$ is not a power of $S(\zeta)$ for any irreducible $\zeta$. Therefore, $\mathcal{C}_{\zeta}=0$ for all $\zeta$. It follows that $\Phi_{L}: \bigoplus_{[\zeta]} \mathcal{C}_{\zeta} / \Delta=0 \rightarrow \mathcal{C} / \Delta$ is not surjective.

On the other hand, $\mathcal{C}$ is right primary decomposable. To prove this, observe that $\Delta_{L}$ is relatively prime to $\mu$ if and only if $L=0$. That is, $\mathcal{C}^{\mu}=0$. Also, $[K] \in \mathcal{C}^{\zeta}$ for $\zeta \neq \lambda, \mu$, and $[J] \in \mathcal{C}^{\zeta}$ for $\zeta \neq \mu, v$. Because $\mathcal{C}$ is generated by $[K]=[J]$, it follows that $\mathcal{C}^{\zeta}=\mathcal{C}$ for $\zeta \neq \mu$. Therefore, $\Phi_{R}: \mathcal{C} / \Delta \rightarrow \bigoplus_{[\zeta]} \mathcal{C} / \mathcal{C}^{\zeta}$ is an isomorphism.

Though the above examples tell us that the bijectivity of $\Phi_{L}$ does not imply the bijectivity of $\Phi_{R}$ or vice versa, the following observations provide partial relationships between the surjectivity and injectivity of $\Phi_{L}$ and $\Phi_{R}$. 


\section{Lemma A.4.}

(1) If $\Phi_{L}$ is surjective, then $\Phi_{R}$ is surjective.

(2) Suppose that $\Phi_{R}$ is injective. Then the following splitting property holds: if $\Delta_{K}$ and $\Delta_{J}$ are relatively prime and $[K]+[J] \equiv 0 \bmod \Delta$, then $[K] \equiv[J] \equiv 0 \bmod \Delta$. In particular, $\Phi_{L}$ is injective.

Proof. (1) The homomorphism $\Phi_{R}$ is surjective if and only if for every given $[K] \in \mathcal{C}$ and $[\lambda] \in \mathbb{P}$ there exists $[J] \in \mathcal{C}$ such that $[J] \equiv[K] \bmod \mathcal{C}^{\lambda}$ and $[J] \equiv 0 \bmod \mathcal{C}^{\mu}$ for all $[\mu] \neq[\lambda]$. By the surjectivity of $\Phi_{L}$ or, equivalently, (LP1), $[K] \equiv\left[K_{1}\right]+\cdots+\left[K_{n}\right] \bmod \Delta$ for some $\left[K_{i}\right] \in \mathcal{C}_{\lambda_{i}}$, where $\left[\lambda_{i}\right] \neq\left[\lambda_{j}\right]$ for $i \neq j$. If $[\lambda]=\left[\lambda_{i}\right]$ for some $i$, then let $[J]=\left[K_{i}\right]$. Otherwise, let $[J]=0$. Then, because $\mathcal{C}_{\lambda_{i}} \subset \mathcal{C}^{\mu}$ whenever $[\mu] \neq\left[\lambda_{i}\right]$, it follows that $[J]$ has the desired property.

(2) Suppose that $\operatorname{gcd}\left(\Delta_{K}, \Delta_{J}\right)=1$ and $[K]+[J] \equiv 0 \bmod \Delta$. If an irreducible $\lambda$ divides $\Delta_{J}$, then $\operatorname{gcd}\left(\lambda, \Delta_{K}\right)=1$ and so $[K] \equiv 0 \bmod \mathcal{C}^{\lambda}$. If $\operatorname{gcd}\left(\lambda, \Delta_{J}\right)=1$, then $[K] \equiv[K]+[J] \equiv 0 \bmod \mathcal{C}^{\lambda}$. So $[K] \equiv 0 \bmod \mathcal{C}^{\lambda}$ for all $\lambda$, and thus $[K] \equiv 0 \bmod \Delta$ by the injectivity of $\Phi_{R}$. The same argument shows $[J] \equiv 0 \bmod \Delta$. From this, (LP2) immediately follows. That is, $\Phi_{L}$ is injective.

Remark A.5. As seen in the last sentence of the above proof of Lemma A.4(1), the composition $\mathcal{C}_{\lambda} / \Delta \hookrightarrow \mathcal{C} / \Delta \rightarrow \mathcal{C} / \mathcal{C}^{\mu}$ is zero if $[\lambda] \neq[\mu]$. Consequently, the composition

$$
\Phi_{R} \circ \Phi_{L}: \bigoplus_{[\lambda] \in \mathbb{P}} \mathcal{C}_{\lambda} / \Delta \longrightarrow \bigoplus_{[\lambda] \in \mathbb{P}} \mathcal{C} / \mathcal{C}^{\lambda}
$$

is the orthogonal direct sum of the compositions $\mathcal{C}_{\lambda} / \Delta \hookrightarrow \mathcal{C} / \Delta \rightarrow \mathcal{C} / \mathcal{C}^{\lambda}$. It follows that $\mathcal{C}_{\lambda} / \Delta \hookrightarrow$ $\mathcal{C} / \Delta \rightarrow \mathcal{C} / \mathcal{C}^{\lambda}$ is an isomorphism for each irreducible $\lambda$ if and only if $\Phi_{R} \circ \Phi_{L}$ is an isomorphism. Consequently, $\mathcal{C}_{\lambda} / \Delta \hookrightarrow \mathcal{C} / \Delta \rightarrow \mathcal{C} / \mathcal{C}^{\lambda}$ is an isomorphism if $\mathcal{C}$ is left primary decomposable and right primary decomposable.

Recall that $\mathcal{C}$ is left primary decomposable if and only if (LP1) and (LP2) hold. The following is a stronger variation of (LP1):

(LP1') Strong existence: for every $K \in \mathbb{K}$, there exist irreducible factors $\lambda_{1}, \ldots, \lambda_{n}$ of $\Delta_{K}$ and $\left[K_{1}\right] \in$ $\mathcal{C}_{\lambda_{1}}, \ldots,\left[K_{n}\right] \in \mathcal{C}_{\lambda_{n}}$ such that $[K] \equiv\left[K_{1}\right]+\cdots+\left[K_{n}\right] \bmod \Delta$.

Definition A.6. We say that $\mathcal{C}=\mathbb{K} / \sim$ is strongly primary decomposable if (LP1') and (LP2) hold.

Proposition A.7. If $\mathcal{C}$ is strongly primary decomposable, then $\mathcal{C}$ is left primary decomposable and right primary decomposable.

Proof. If $\mathcal{C}$ is strongly primary decomposable, $\mathcal{C}$ is obviously left primary decomposable. Also, $\Phi_{R}$ is surjective by Lemma A.4(1). So it remains to show that $\Phi_{R}$ is injective.

Suppose that $[K]$ lies in $\mathcal{C}^{\lambda}$ for all irreducibles $\lambda$. The goal is to show that $[K] \in \Delta$. We claim that the given $[K]$ can be assumed to lie in $\mathcal{C}_{\mu}$ for some $[\mu] \in \mathbb{P}$. To prove this, first use (LP1) (or the surjectivity of $\Phi_{L}$ ) to write $[K] \equiv\left[K_{1}\right]+\cdots+\left[K_{n}\right] \bmod \Delta$ for some $K_{i} \in \mathcal{C}_{\lambda_{i}}$, where $\left[\lambda_{i}\right] \neq\left[\lambda_{j}\right]$ for $i \neq j$. Fix $i$. We have that $\left[K_{i}\right] \equiv[K]-\sum_{j \neq i}\left[K_{j}\right] \bmod \Delta,\left[K_{j}\right] \in \mathcal{C}_{\lambda_{j}} \subset \mathcal{C}^{\lambda_{i}}$ for all $j \neq i$, and $[K] \in \mathcal{C}^{\lambda_{i}}$. It follows that $\left[K_{i}\right] \in \mathcal{C}^{\lambda_{i}}$. Because $\left[K_{i}\right] \in \mathcal{C}_{\lambda_{i}},\left[K_{i}\right] \in \mathcal{C}^{\lambda}$ for $[\lambda] \neq\left[\lambda_{i}\right]$. So, $\left[K_{i}\right]$ lies in $\mathcal{C}^{\lambda}$ for all $\lambda$. Note that it suffices to show that $\left[K_{i}\right] \in \Delta$ for all $i$, to conclude that $[K] \in \Delta$. This proves the claim.

Now, fix an irreducible $\mu$. Suppose that $[K] \in \mathcal{C}_{\mu}$ and $[K] \in \mathcal{C}^{\lambda}$ for all $\lambda$. Because $[K] \in \mathcal{C}^{\mu}$, we have $[K]=[J]$ for some $J \in \mathbb{K}$ such that $\operatorname{gcd}\left(\Delta_{J}, \mu\right)=1$. By $\left(\mathrm{LP}^{\prime}\right),[J] \equiv\left[J_{1}\right]+\cdots\left[J_{m}\right] \bmod \Delta$ for some $\left[J_{k}\right] \in \mathcal{C}_{\mu_{k}}$, where the $\mu_{k}$ are irreducible factors of $\Delta_{J}$. In particular, every $\mu_{k}$ is relatively prime to $\mu$. Also, $\mu_{k}^{*}$ is relatively prime to $\mu$, because $\mu_{k}^{*}$ divides $\Delta_{J}^{*} \doteq \Delta_{J}$, which is relatively prime to $\mu$. So $\left[\mu_{k}\right] \neq[\mu]$ in $\mathbb{P}$ for all $k$. Because $-[K]+\left[J_{1}\right]+\cdots+\left[J_{m}\right] \in \Delta$ and $[K] \in \mathcal{C}_{\mu}$, it follows that $[K]$ lies in $\Delta$ by (LP2). 


\section{A.2. Extensions}

Suppose that $(\mathbb{K}, \sim, \chi)$ is as in Subsection A.1 and $\mathcal{A}$ is a subgroup of $\mathcal{C}=\mathbb{K} / \sim$. We have $\mathcal{A}=\mathbb{A} / \sim$ for the submonoid $\mathbb{A}:=\{K \in \mathbb{K} \mid[K] \in \mathcal{A}\}$. Or, conversely, if $\mathbb{A}$ is a submonoid of $\mathbb{K}$ such that $K+J \sim 0$ and $K \in \mathbb{A}$ imply $J \in \mathbb{A}$, then $\mathcal{A}:=\mathbb{A} / \sim$ is a subgroup of $\mathcal{C}$. Let $\mathcal{G}=\mathcal{C} / \mathcal{A}$ be the quotient group. We have $\mathcal{G}=\mathbb{K} / \approx$, where the equivalence relation $\approx$ is defined by $K \approx J$ if and only if $K \sim J+L$ for some $L \in \mathbb{A}$.

We will relate primary decompositions of $\mathcal{A}, \mathcal{C}$ and $\mathcal{G}$. To avoid confusion, for $\mathcal{A}, \mathcal{C}$ and $\mathcal{G}$ respectively, denote by $\Delta(\mathcal{A}), \Delta(\mathcal{C})$ and $\Delta(\mathcal{G})$ their subgroups of classes represented by $K$ with $\Delta_{K} \doteq 1$. We have $\Delta(\mathcal{A})=\Delta(\mathcal{C}) \cap \mathcal{A}$ and $\Delta(\mathcal{G})=(\Delta(\mathcal{C})+\mathcal{A}) / \mathcal{A} \subset \mathcal{G}=\mathcal{C} / \mathcal{A}$. It is straightforward to verify that the exact sequence

$$
0 \longrightarrow \mathcal{A} \rightarrow \mathcal{C} \longrightarrow \mathcal{G} \longrightarrow 0
$$

gives rise to an exact sequence

$$
0 \longrightarrow \Delta(\mathcal{A}) \longrightarrow \Delta(\mathcal{C}) \longrightarrow \Delta(\mathcal{G}) \longrightarrow 0
$$

and induces the following exact sequences for all irreducibles $\lambda$ in $\mathbb{Q}\left[t^{ \pm 1}\right]$ :

$$
\begin{aligned}
& 0 \longrightarrow \mathcal{A}_{\lambda} \longrightarrow \mathcal{C}_{\lambda} \longrightarrow \mathcal{G}_{\lambda} \longrightarrow 0 \\
& 0 \longrightarrow \mathcal{A}^{\lambda} \longrightarrow \mathcal{C}^{\lambda} \longrightarrow \mathcal{G}^{\lambda} \longrightarrow 0
\end{aligned}
$$

Consequently, rows of the following commutative diagram are exact:

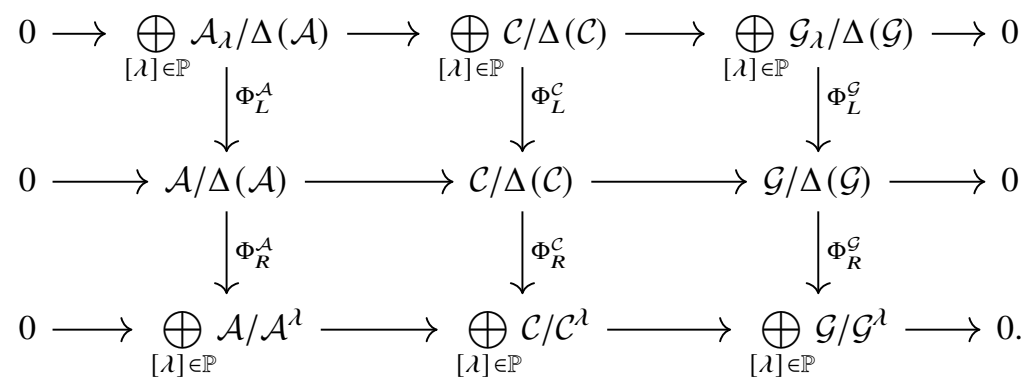

For $\bullet=L$ and $R,(\mathrm{~A} .1)$ gives rise to an exact sequence

$$
\begin{aligned}
0 \longrightarrow \operatorname{Ker} \Phi_{\bullet}^{\mathcal{A}} & \longrightarrow \operatorname{Ker} \Phi_{\bullet}^{\mathcal{C}} \longrightarrow \operatorname{Ker} \Phi_{\bullet}^{\mathcal{G}} \\
& \longrightarrow \operatorname{Coker} \Phi_{\bullet}^{\mathcal{A}} \longrightarrow \operatorname{Coker} \Phi_{\bullet}^{\mathcal{C}} \longrightarrow \operatorname{Coker} \Phi_{\bullet}^{\mathcal{G}} \longrightarrow 0
\end{aligned}
$$

by the snake lemma. It follows that $\Phi_{\bullet}^{\mathcal{C}}$ is an isomorphism if and only if $\Phi_{\bullet}^{\mathcal{A}}$ is injective, $\Phi_{\bullet}^{\mathcal{G}}$ is surjective and the connecting map $\operatorname{Ker} \Phi_{\bullet}^{\mathcal{G}} \rightarrow \operatorname{Coker} \Phi_{\bullet}^{\mathcal{A}}$ is an isomorphism. The following is an immediate consequence.

Theorem A.8. The group $\mathcal{C}$ is left (respectively right) primary decomposable if both $\mathcal{A}$ and $\mathcal{G}$ are left (respectively right) primary decomposable.

Theorem A.9. The group $\mathcal{C}$ is strongly primary decomposable if both $\mathcal{A}$ and $\mathcal{G}$ are strongly primary decomposable.

Proof. Suppose that $\mathcal{A}$ and $\mathcal{G}$ are strongly primary decomposable. Then by Proposition A.7 and Theorem A.8, $\mathcal{C}$ satisfies the uniqueness condition (LP2). So, it remains to verify the strong existence condition $\left(\mathrm{LP} 1^{\prime}\right)$ for $\mathcal{C}$. 
Fix $[K] \in \mathcal{C}$. Because $\mathcal{G}$ satisfies $\left(\mathrm{LP}^{\prime}\right)$, we have $[K] \equiv\left[J_{1}\right]+\cdots+\left[J_{n}\right] \bmod \Delta(\mathcal{C})+\mathcal{A}$ for some $\left[J_{1}\right] \in \mathcal{C}_{\lambda_{1}}, \ldots,\left[J_{n}\right] \in \mathcal{C}_{\lambda_{n}}$, where each $\lambda_{i}$ is an irreducible factor of $\Delta_{K}$ and $\Delta_{J_{i}}$ is a power of $S\left(\lambda_{i}\right)$. So, for some $[J] \in \mathcal{C}$ with $\Delta_{J} \doteq 1$, we have

$$
[K]-\left(\left[J_{1}\right]+\cdots+\left[J_{n}\right]\right)+[J] \in \mathcal{A} .
$$

Using property $(\Delta 3)$, choose $J_{1}^{\prime}, \ldots, J_{n}^{\prime}$ such that $\left[J_{i}^{\prime}\right]=-\left[J_{i}\right]$ and $\Delta_{J_{i}^{\prime}} \doteq \Delta_{J_{i}}$, and let $L=K+J_{1}^{\prime}+\cdots+$ $J_{n}^{\prime}+J$. Then $[L] \in \mathcal{A}$ by (A.3) and $\Delta_{L} \doteq \Delta_{K} \cdot \prod \Delta_{J_{i}}$ by $(\Delta 2)$. In particular, irreducibles dividing $\Delta_{L}$ divide $\Delta_{K}$. Because $\mathcal{A}$ satisfies $\left(\mathrm{LP} 1^{\prime}\right)$, there is a decomposition

$$
[L] \equiv\left[L_{1}\right]+\cdots+\left[L_{m}\right] \bmod \Delta(\mathcal{A})=\mathcal{A} \cap \Delta(\mathcal{C}),
$$

where $\left[L_{j}\right] \in \mathcal{A}_{\mu_{j}}$ and each $\mu_{j}$ is an irreducible factor of $\Delta_{K}$. It follows that

$$
[K] \equiv\left[J_{1}\right]+\cdots+\left[J_{n}\right]+\left[L_{1}\right]+\cdots+\left[L_{m}\right] \bmod \Delta(\mathcal{C}) .
$$

Recall that $\left[J_{i}\right] \in \mathcal{C}_{\lambda_{i}},\left[L_{j}\right] \in \mathcal{A}_{\mu_{j}} \subset \mathcal{C}_{\mu_{j}}$ and $\lambda_{i}$ and $\mu_{j}$ are factors of $\Delta_{K}$. So, (LP1') is satisfied for the given $[K] \in \mathcal{C}$.

\section{A.3. Knot concordance and primary decomposition}

\section{Algebraic concordance over $\mathbb{Q}$}

Levine's work on knot concordance provides an algebraic analogue of the knot concordance group, which is now called the algebraic concordance group [35,34]. The algebraic concordance group over $\mathbb{Q}$ is known to be left and right primary decomposable in our sense. We describe this using Blanchfield linking forms over $\mathbb{Q}\left[t^{ \pm 1}\right]$, whereas Levine's original papers [35, 34] use Seifert matrices.

Let $\mathbb{Q}(t)$ be the rational function field. A $\left(\mathbb{Q}(t) / \mathbb{Q}\left[t^{ \pm 1}\right]\right)$-valued linking form is defined to be a map $B: V \times V \rightarrow \mathbb{Q}(t) / \mathbb{Q}\left[t^{ \pm 1}\right]$, with $V$ a finitely generated $\mathbb{Q}\left[t^{ \pm 1}\right]$-module such that $V \otimes_{\mathbb{Q}\left[t^{ \pm 1}\right]} \mathbb{Q}(t)=0$, which is sesquilinear and nonsingular. That is, $B(x, y)=B(y, x)^{*}, y \mapsto B(x,-)$ is $\mathbb{Q}\left[t^{ \pm 1}\right]$-linear for all $x \in V$, and the adjoint $V \rightarrow \operatorname{Hom}_{\mathbb{Q}\left[t^{ \pm 1}\right]}\left(V, \mathbb{Q}(t) / \mathbb{Q}\left[t^{ \pm 1}\right]\right)$ is an isomorphism. Let $\mathbb{K}$ be the monoid of $\left(\mathbb{Q}(t) / \mathbb{Q}\left[t^{ \pm 1}\right]\right)$-valued linking forms (under direct sum operation), and define $\chi: \mathbb{K} \rightarrow \mathbb{Q}\left[t^{ \pm 1}\right] / \doteq$ by $\chi(B)=\Delta_{B}(t)$, where the Alexander polynomial $\Delta_{B}(t)$ is defined to be the order of the torsion module $V$ over $\mathbb{Q}\left[t^{ \pm 1}\right]$. A linking form $B$ is metabolic if there is a submodule $P \subset V$ such that $P=\{x \in V \mid B(x, P)=0\}$. We say that $B$ and $B^{\prime}$ are Witt equivalent if there are metabolic linking forms $H$ and $H^{\prime}$ such that the orthogonal sums $B \oplus H$ and $B^{\prime} \oplus H$ are isomorphic. The set $\mathcal{G}$ of Witt equivalence classes of linking forms is an abelian group under orthogonal sum. ${ }^{1}$ The conditions $(\Delta 1)$, $(\Delta 2)$ and $(\Delta 3)$ stated in Subsection A.1 hold. By definition, $\Delta_{B}(t)$ is trivial if and only if $V$ is trivial. It follows that the subgroup $\Delta=\left\{[B] \in \mathcal{G} \mid \Delta_{B} \doteq 1\right\}$ is trivial.

The following result is essentially due to Levine [34].

Theorem A.10 (Levine [34]). The group $\mathcal{G}$ is left and right primary decomposable.

$$
\bigoplus_{[\lambda]} \mathcal{G}_{\lambda} \stackrel{\Phi_{L}}{\cong} \mathcal{G} \underset{\cong}{\stackrel{\Phi_{R}}{\longrightarrow}} \bigoplus_{[\lambda]} \mathcal{G} / \mathcal{G}^{\lambda}
$$

Indeed, $\mathcal{G}$ is strongly primary decomposable. An elegant generalisation of Levine's Theorem A.10 to the case of the group ring of a (noncommutative) free group was developed in work of Sheiham [43, Section 3]. See also [42].

\footnotetext{
${ }^{1}$ For the study of knots, often the Witt group of linking forms $B$ satisfying $\Delta_{B}(1) \Delta_{B}(-1) \neq 0$ is considered. One can also consider (-1)-linking forms, which satisfy $B(x, y)=-B(y, x)^{*}$ instead of $B(x, y)=B(y, x)^{*}$. Variations of Theorem A.10 hold for these cases as well.
} 


\section{Algebraic concordance over $\mathbb{Z}$}

For a knot $K$ in $S^{3}$, the Blanchfield form [1] $B_{K}$ defined on the Alexander module $H_{1}\left(S^{3} \backslash K\right.$; $\left.\mathbb{Q}\left[t^{ \pm 1}\right]\right)$ is a linking form in the above sense. We have $\Delta_{K}=\Delta_{B_{K}}$. The association $K \mapsto B_{K}$ induces a homomorphism of the (topological and smooth) knot concordance group into $\mathcal{G}$. The image of this homomorphism is characterised as follows. By replacing $\left(\mathbb{Q}\left[t^{ \pm 1}\right], \mathbb{Q}(t)\right)$ with $\left(\mathbb{Z}\left[t^{ \pm 1}\right], S^{-1} \mathbb{Z}\left[t^{ \pm 1}\right]\right)$ where $S=\left\{f(t) \in \mathbb{Z}\left[t^{ \pm 1}\right] \mid f(1)= \pm 1\right\}$, one constructs an integral analogue of $\mathcal{G}$, say, $\mathcal{G}(\mathbb{Z})$. It is known that the natural map $\mathcal{G}(\mathbb{Z}) \rightarrow \mathcal{G}$ is injective [34], and the subgroup $\mathcal{G}(\mathbb{Z})$ of $\mathcal{G}$ is exactly the image of the concordance group of knots in $S^{3}$. A knot $K$ such that $\left[B_{K}\right]=0$ in $\mathcal{G}(\mathbb{Z}) \subset \mathcal{G}$ is said to be algebraically slice.

Levine showed that both $\mathcal{G}$ and $\mathcal{G}(\mathbb{Z})$ are isomorphic to $\mathbb{Z}^{\infty} \oplus\left(\mathbb{Z}_{2}\right)^{\infty} \oplus\left(\mathbb{Z}_{4}\right)^{\infty}$ [34]. The difference of the structures of $\mathcal{G}$ and $\mathcal{G}(\mathbb{Z})$ was studied in work of Stoltzfus [44]. Among his main results, it was shown that $\Phi_{L}: \bigoplus_{[\lambda]} \mathcal{G}(\mathbb{Z})_{\lambda} \rightarrow \mathcal{G}(\mathbb{Z})$ is not surjective (whereas it is injective due to Levine's work [35]). In particular, $\mathcal{G}(\mathbb{Z})$ is not left primary decomposable. (To the author's knowledge, it was not addressed in the literature whether $\mathcal{G}(\mathbb{Z})$ is right primary decomposable.)

\section{Topological knot concordance and algebraically slice knots}

Let $\mathbb{K}$ be the set of isotopy classes of oriented knots in $S^{3}$, with connected sum as a monoid operation. If $K, K^{\prime} \in \mathbb{K}$ are topologically concordant, write $K \sim K^{\prime}$. The set of equivalence classes $\mathcal{C}^{\text {top }}=\mathbb{K} / \sim$ is the topological knot concordance group. Define $\chi: \mathbb{K} \rightarrow \mathbb{Q}\left[t^{ \pm 1}\right] / \doteq$ by $\chi(K)=\Delta_{K}(t)$, the Alexander polynomial of $K$. The assumptions $(\Delta 1),(\Delta 2)$ and $(\Delta 3)$ in Subsection A.1 are standard properties of the Alexander polynomial. By work of Freedman [21], the subgroup $\Delta=\left\{[K] \in \mathcal{C}^{\text {top }} \mid \Delta_{K} \doteq 1\right\}$ is trivial.

Let $\mathcal{A}^{\text {top }}$ be the topological concordance group of algebraically slice knots. Then, because

$$
0 \longrightarrow \mathcal{A}^{\text {top }} \longrightarrow \mathcal{C}^{\text {top }} \longrightarrow \mathcal{G}^{\mathbb{Z}} \longrightarrow 0
$$

is exact, $\mathcal{C}^{\text {top }}$ is right primary decomposable if so are both $\mathcal{A}^{\text {top }}$ and $\mathcal{G}^{\mathbb{Z}}$. Regarding left primary decomposability, because $\bigoplus_{[\lambda]} \mathcal{G}_{\lambda}^{\mathbb{Z}} \rightarrow \mathcal{G}^{\mathbb{Z}}$ is not surjective due to Stoltzfus [44], $\Phi_{L}^{\text {top }}: \bigoplus_{[\lambda]} \mathcal{C}_{\lambda}^{\text {top }} \rightarrow \mathcal{C}^{\text {top }}$ is not surjective by the exact sequence (A.2). In particular, $\mathcal{C}^{\text {top }}$ is not left primary decomposable. The following appear to be interesting.

\section{Question A.11.}

(1) Is $\Phi_{L}^{\text {top }}: \bigoplus_{[\lambda]} \mathcal{C}_{\lambda}^{\text {top }} \rightarrow \mathcal{C}^{\text {top }}$ injective?

(2) Is $\mathcal{C}^{\text {top }}$ right primary decomposable?

By Lemma A.4(2), an affirmative answer to Question A.11(2) implies that the answer to Question A.11(1) is affirmative and that the splitting property in Lemma A.4(2) holds.

In the literature, there are related results that provide affirmative answers to the following splitting question for certain concordance invariants (or obstructions): if $K$ and $J$ have relatively prime Alexander polynomials and if the invariant vanishes for $K \# J$, then does the invariant vanish for each of $K$ and $J$ ? See work of S.-G. Kim [28], which addresses the case of Casson-Gordon invariants, and work of and S.-G. Kim and T. Kim [29, 30] on $L^{2}$-signature defects. These may be viewed as evidence supporting an affirmative answer to the injectivity part for $\Phi_{R}^{\text {top }}$ in Question A.11(2), which implies the splitting property stated in Lemma A.4(2). Also, T. Kim [32] proved results related to primary decomposition structures in $\mathcal{A}^{\text {top }}$.

\section{Smooth concordance and topologically slice knots}

Let $\mathbb{K}$ and $\chi$ be as above but now write $K \sim K^{\prime}$ if $K$ and $K^{\prime}$ are smoothly concordant. Then $\mathcal{C}^{\text {sm }}=\mathbb{K} / \sim$ is the smooth knot concordance group. 
Similar to the case of $\mathcal{C}^{\text {top }}$, the homomorphism $\Phi_{L}^{\mathrm{sm}}: \bigoplus_{[\lambda]} \mathcal{C}_{\lambda}^{\mathrm{sm}} / \Delta \rightarrow \mathcal{C}^{\text {sm }} / \Delta$ is not surjective because $\bigoplus_{[\lambda]} \mathcal{G}_{\lambda}^{\mathbb{Z}} \rightarrow \mathcal{G}^{\mathbb{Z}}$ is not surjective. So $\mathcal{C}^{\text {sm }}$ is not left primary decomposable.

\section{Question A.12.}

(1) Is $\Phi_{L}^{\mathrm{sm}}: \bigoplus_{[\lambda]} \mathcal{C}_{\lambda}^{\mathrm{sm}} / \Delta \rightarrow \mathcal{C}^{\mathrm{sm}} / \Delta$ injective?

(2) Is $\mathcal{C}^{\mathrm{sm}}$ right primary decomposable?

Again by Lemma A.4(2), an affirmative answer to Question A.12(2) implies that the answer to Question A.12(1) is affimative and that the splitting property stated in Lemma A.4(2) holds.

The smooth concordance group of topologically slice knots $\mathcal{T}$ is the kernel of the natural homomorphism $\mathcal{C}^{\mathrm{sm}} \rightarrow \mathcal{C}^{\text {top }}$. By Theorem A.8, $\mathcal{C}^{\text {sm }}$ is right primary decomposable if both $\mathcal{T}$ and $\mathcal{C}^{\text {top }}$ are right primary decomposable.

Question 1.1 in the Introduction asks whether $\mathcal{T}$ is left and right primary decomposable in the sense of Definition A.1, and Theorem A provides supporting evidence for affirmative answers.

Recall that the definition in Subsection A.1 says that $[K] \in \mathcal{T}_{\lambda}$ if $\Delta_{K} \doteq S(\lambda)^{k}$ for some $k \geq 0$. For topologically slice $K, \Delta_{K} \doteq f f^{*}$ for some $f \in \mathbb{Q}\left[t^{ \pm 1}\right]$, due to Fox and Milnor [20]. From this it follows that $\Delta_{K} \doteq S(\lambda)^{k}$ for some $k \geq 0$ if and only if $\Delta_{K} \doteq\left(\lambda \lambda^{*}\right)^{\ell}$ for some $\ell \geq 0$. Thus, the definition of $\mathcal{T}_{\lambda}$ in Subsection A.1 agrees with that in Subsection 1.1.

Livingston informed us that the techniques of [25] can be used to show the following: there are nonassociate self-dual irreducible polynomials $\lambda_{1}, \lambda_{2}$ and $\lambda_{3}$ and a topologically slice knot $K$ with $\Delta_{K}=\left(\lambda_{1} \lambda_{2} \lambda_{3}\right)^{2}$ that is not smoothly concordant to a connected sum $K_{1} \# K_{2} \# K_{3}$ for any knots $K_{1}, K_{2}$ and $K_{3}$ with $\Delta_{K_{i}}$ a power of $\lambda_{i}$. It says that $\mathcal{T}$ does not satisfy the strong existence condition (LP1'). This method does not provide counterexamples to the left/right primary decomposability of $\mathcal{T}$.

\section{Filtrations of the knot concordance groups}

For the bipolar filtration $\left\{\mathcal{T}_{n}\right\}$ of $\mathcal{T}$ defined by Cochran, Harvey and Horn [11], Question 1.2 in the Introduction asks whether the associated graded $\operatorname{gr}_{n}(\mathcal{T})=\mathcal{T}_{n} / \mathcal{T}_{n+1}$ is left and right primary decomposable in the sense of Definition A.1. Theorem $C$ supports an affirmative answer, by presenting a large subgroup that is left and right (indeed strongly) primary decomposable into infinitely many infinite rank primary parts.

In addition, whether $\mathcal{T}_{n}$ is left/right primary decomposable appears to be an interesting problem. By Theorems A.8 and A.9, $\mathcal{T}_{n}$ is left/right primary decomposable if so are both $\mathcal{T}_{n+1}$ and $\operatorname{gr}_{n}(\mathcal{T})$.

In [16], Cochran, Orr and Teichner introduced a descending filtration

$$
\{0\} \subset \cdots \subset \mathcal{F}_{n .5} \subset \mathcal{F}_{n} \subset \cdots \subset \mathcal{F}_{1} \subset \mathcal{F}_{0.5} \subset \mathcal{F}_{0} \subset \mathcal{C}^{\text {top }}
$$

of the topological knot concordance group $\mathcal{C}^{\text {top }}$. A knot $K$ represents an element of $\mathcal{F}_{h}\left(h \in \frac{1}{2} \mathbb{Z}_{\geq 0}\right)$ if $K$ is $h$-solvable in the sense of [16, Definitions 8.5 and 8.7].

Question A.13. Are $\mathcal{F}_{h}$ and $\mathcal{F}_{h} / \mathcal{F}_{h+0.5}$ left and/or right primary decomposable?

Once again by Theorem A.8, $\mathcal{F}_{h}$ is left/right primary decomposable if so are both $\mathcal{F}_{h+0.5}$ and $\mathcal{F}_{n} / \mathcal{F}_{h+0.5}$. For integers $n>0$, it is unknown whether $\mathcal{F}_{n .5} / \mathcal{F}_{n+1}$ is nontrivial. Recently, Davis, Martin, Otto and Park showed that elements in $\mathcal{F}_{0.5}$ represented by a genus one knot are contained in $\mathcal{F}_{1}$ [19]. For the other half of the associated graded $\mathcal{F}_{n} / \mathcal{F}_{n .5}$, Cochran, Harvey and Leidy provided strong evidence that supports the conjecture that the associated graded $\mathcal{F}_{n} / \mathcal{F}_{n .5}$ is right primary decomposable for all integers $n \geq 0[14,13]$. Indeed, they proposed a highly refined primary decomposition conjecture for the associated graded $\mathcal{F}_{n} / \mathcal{F}_{n .5}$, using noncommutative localisations [14, p. 444], and they showed that refined primary parts reveal interesting structures [14, 13]. We remark that aforementioned earlier works of S.-G. Kim and T. Kim [28, 29] also provide supporting evidence for the case of $\mathcal{F}_{1} / \mathcal{F}_{1.5}$. 


\section{A.4. Rational homology 3-spheres and primary decomposition}

Let $\Theta_{\mathbb{Q}}^{\text {top }}$ and $\Theta_{\mathbb{Q}}^{\text {sm }}$ be the topological and smooth rational homology cobordism groups of rational homology 3-spheres, respectively. For a rational homology 3-sphere $Y$, let $\Delta_{Y}=\left|H_{1}(Y)\right| \in \mathbb{Z}$, the order of the first homology with integral coefficients. The association $\chi: Y \mapsto \Delta_{Y}=\left|H_{1}(Y)\right|$ satisfies conditions $(\Delta 1),(\Delta 2)$ and $(\Delta 3)$. So, our general definition of primary decomposition applies to $\Theta_{\mathbb{Q}}^{\text {top }}$ and $\Theta_{\mathbb{Q}}^{\mathrm{sm}}$.

For the topological case, the subgroup $\Delta=\left\{[Y] \in \Theta_{\odot}^{\text {top }} \mid H_{1}(Y)=0\right\}$ is trivial, because every integral homology 3-sphere bounds a contractible compact 4-manifold [22, Section 9.3C]. So, the left and right primary decompositions concern the homomorphisms

$$
\Phi_{L}: \bigoplus_{p}\left(\Theta_{\mathbb{Q}}^{\text {top }}\right)_{p} \longrightarrow \Theta_{\mathbb{Q}}^{\text {top }}, \quad \Phi_{R}: \Theta_{\mathbb{Q}}^{\text {top }} \longrightarrow \bigoplus_{p} \Theta_{\mathbb{Q}}^{\text {top }} /\left(\Theta_{\mathbb{Q}}^{\text {top }}\right)^{p},
$$

where $p$ varies over primes in $\mathbb{Z}$. Here, the primary part $\left(\Theta_{\mathbb{Q}}^{\text {top }}\right)_{p}$ is generated by $\mathbb{Z}\left[\frac{1}{p}\right]$-homology spheres, and $\left(\Theta_{\mathbb{Q}}^{\text {top }}\right)^{p}$ is generated by $\mathbb{Z}_{p}$-homology spheres.

The linking form $L_{Y}: H_{1}(Y) \times H_{1}(Y) \rightarrow \mathbb{Q} / \mathbb{Z}$ of a rational homology 3-sphere $Y$ gives fundamental information. Algebraically, a $(\mathbb{Q} / \mathbb{Z})$-valued linking form is a nonsingular symmetric bilinear form $L: A \times A \rightarrow \mathbb{Q} / \mathbb{Z}$ with $A$ a finite abelian group. The Witt group of $(\mathbb{Q} / \mathbb{Z})$-valued linking forms, which we denote by $W(\mathbb{Q} / \mathbb{Z})$, is defined in the standard manner. There is a homomorphism $\Theta_{\mathbb{Q}}^{\text {top }} \rightarrow W(\mathbb{Q} / \mathbb{Z})$ that sends the class of a rational homology sphere $Y$ to the Witt class of the associated linking form $L_{Y}$. This is surjective due to [27]. The following is a well-known conjecture [31, p. 4].

Conjecture A.14. The homomorphism $\Theta_{\mathbb{Q}}^{\text {top }} \rightarrow W(\mathbb{Q} / \mathbb{Z})$ is an isomorphism.

Also, it is a standard fact that the Witt group $W(\mathbb{Q} / \mathbb{Z})$ is left and right primary decomposable. (Indeed, the strong existence condition (LP1') is also satisfied.) The primary part $W(\mathbb{Q} / \mathbb{Z})_{p}$ is the Witt group of linking forms defined on $p$-torsion finite abelian groups. So, an affirmative answer to the above conjecture implies that $\Theta^{\text {top }}$ is left and right primary decomposable.

For the smooth case, the left and right primary decompositions concern the homomorphisms

$$
\Phi_{L}: \bigoplus_{p}\left(\Theta_{\mathbb{Q}}^{\mathrm{sm}}\right)_{p} / \Delta \longrightarrow \Theta_{\mathbb{Q}}^{\mathrm{sm}} / \Delta, \quad \Phi_{R}: \Theta_{\mathbb{Q}}^{\mathrm{sm}} / \Delta \longrightarrow \bigoplus_{p} \Theta_{\mathbb{Q}}^{\mathrm{sm}} /\left(\Theta_{\mathbb{Q}}^{\mathrm{sm}}\right)^{p},
$$

where $\Delta \subset \Theta_{\mathbb{Q}}^{\mathrm{sm}}$ is the subgroup generated by the classes of integral homology 3-spheres. A result of S.-G. Kim and Livingston [31, Proposition, p. 184] says that this $\Phi_{L}$ is not surjective, and thus $\Theta_{\mathbb{Q}}^{\mathrm{sm}}$ is not left primary decomposable. We have the following questions.

\section{Question A.15.}

(1) Is $\Phi_{L}: \bigoplus_{p}\left(\Theta_{\mathbb{Q}}^{\mathrm{sm}}\right)_{p} / \Delta \rightarrow \Theta_{\mathbb{Q}}^{\mathrm{sm}} / \Delta$ injective?

(2) Is $\Theta_{\mathbb{Q}}^{\text {sm }}$ right primary decomposable?

By Theorem A.8, $\Theta_{\mathbb{O}}^{\text {sm }}$ is right primary decomposable if Conjecture A.14 is true and the rational homology cobordism group of rational homology 3-spheres bounding a topological rational homology 4-ball is right primary decomposable.

\footnotetext{
Acknowledgements. The author is indebted to Min Hoon Kim, Se-Goo Kim and Taehee Kim for discussions that led him to prove the results described in the Introduction. The author thanks Chuck Livingston for his extremely helpful comments. Many results in this article were obtained during the author's visit to Max Planck Institute for Mathematics in 2017-2018. The author is grateful to the institute and Peter Teichner for the invitation and hospitality. Finally, the author thanks an anonymous referee for comments that were very helpful in improving the exposition.
} 


\section{References}

[1] Richard C. Blanchfield, 'Intersection theory of manifolds with operators with applications to knot theory', Ann. Math. 65(2) (1957), 340-356.

[2] Jae Choon Cha, 'The structure of the rational concordance group of knots', Mem. Amer. Math. Soc. 189(885) (2007), x+95.

[3] Jae Choon Cha, 'Amenable $\mathrm{L}^{2}$-theoretic methods and knot concordance', Int. Math. Res. Not. IMRN 17 (2014), 4768-4803.

[4] Jae Choon Cha, 'Symmetric Whitney tower cobordism for bordered 3-manifolds and links', Trans. Amer. Math. Soc. 366(6) (2014), 3241-3273.

[5] Jae Choon Cha, 'A topological approach to Cheeger-Gromov universal bounds for von Neumann $\rho$-invariants', Comm. Pure Appl. Math. 69(6) (2016), 1154-1209.

[6] Jae Choon Cha and Min Hoon Kim, 'The bipolar filtration of topologically slice knots', Adv. Math. 388 (2021), 107868. https://doi.org/10.1016/j.aim.2021.107868.

[7] Jae Choon Cha and Ki Hyoung Ko, 'Signatures of links in rational homology homology spheres', Topology 41 (2002), 1161-1182.

[8] Jae Choon Cha and Kent E. Orr, 'L ${ }^{2}$-signatures, homology localization, and amenable groups', Comm. Pure Appl. Math. 65 (2012), 790-832.

[9] Stanley Chang and Shmuel Weinberger, 'On invariants of Hirzebruch and Cheeger-Gromov', Geom. Topol. 7 (2003), 311-319. https://doi.org/10.2140/gt.2003.7.311.

[10] Jeff Cheeger and Mikhael Gromov, 'Bounds on the von Neumann dimension of $\mathrm{L}^{2}$-cohomology and the Gauss-Bonnet theorem for open manifolds', J. Differential Geom. 21(1) (1985), 1-34.

[11] Tim D. Cochran, Shelly Harvey and Peter Horn, 'Filtering smooth concordance classes of topologically slice knots', Geom. Topol. 17(4) (2013), 2103-2162.

[12] Tim D. Cochran, Shelly Harvey and Constance Leidy, 'Knot concordance and higher-order Blanchfield duality', Geom. Topol. 13(3) (2009), 1419-1482.

[13] Tim D. Cochran, Shelly Harvey and Constance Leidy, '2-Torsion in the n-solvable filtration of the knot concordance group', Proc. Lond. Math. Soc. (3) 102(2) (2011), 257-290.

[14] Tim D. Cochran, Shelly Harvey and Constance Leidy, 'Primary decomposition and the fractal nature of knot concordance', Math. Ann. 351(2) (2011), 443-508.

[15] Tim D. Cochran and Peter Horn, 'Structure in the bipolar filtration of topologically slice knots', Algebr. Geom. Topol. 15(1) (2015), 415-428.

[16] Tim D. Cochran, Kent E. Orr and Peter Teichner, 'Knot concordance, Whitney towers and L ${ }^{2}$-signatures', Ann. Math. (2) 157(2) (2003), 433-519.

[17] Tim D. Cochran, Kent E. Orr and Peter Teichner, 'Structure in the classical knot concordance group', Comment. Math. Helv. 79(1) (2004), 105-123.

[18] Tim D. Cochran and Peter Teichner, 'Knot concordance and von Neumann $\rho$-invariants', Duke Math. J. 137(2) (2007), 337-379.

[19] Christopher W. Davis, Taylor Martin, Carolyn Otto and JungHwan Park, 'Every genus one algebraically slice knot is 1-solvable', Trans. Amer. Math. Soc. 372(5) (2019), 3063-3082.

[20] Ralph H. Fox and John W. Milnor, 'Singularities of 2-spheres in 4-space and cobordism of knots', Osaka J. Math. 3, (1966), 257-267.

[21] Michael H. Freedman, 'The disk theorem for four-dimensional manifolds', in Proceedings of the International Congress of Mathematicians, Vols. 1, 2 (PWN, Warsaw, 1984), 647-663.

[22] Michael H. Freedman and Frank Quinn, Topology of 4-Manifolds, Princeton Mathematical Series, Vol. 39 (Princeton University Press, Princeton, NJ, 1990).

[23] Shelly Harvey, 'Homology cobordism invariants and the Cochran-Orr-Teichner filtration of the link concordance group', Geom. Topol. 12 (2008), 387-430.

[24] Matthew Hedden, Se-Goo Kim and Charles Livingston, 'Topologically slice knots of smooth concordance order two', J. Differential Geom. 102(3) (2016), 353-393.

[25] Matthew Hedden, Charles Livingston and Daniel Ruberman, 'Topologically slice knots with nontrivial Alexander polynomial', Adv. Math. 231(2) (2012), 913-939.

[26] James Howie and Hans Rudolf Schneebeli, 'Homological and topological properties of locally indicable groups', Manuscripta Math. 44(1-3) (1983), 71-93.

[27] Akio Kawauchi and Sadayoshi Kojima, 'Algebraic classification of linking pairings on 3-manifolds', Math. Ann. 253(1) (1980), 29-42.

[28] Se-Goo Kim, 'Polynomial splittings of Casson-Gordon invariants', Math. Proc. Cambridge Philos. Soc. 138(1) (2005), $59-78$.

[29] Se-Goo Kim and Taehee Kim, 'Polynomial splittings of metabelian von Neumann rho-invariants of knots', Proc. Amer. Math. Soc. 136(11) (2008), 4079-4087.

[30] Se-Goo Kim and Taehee Kim, 'Splittings of von Neumann rho-invariants of knots', J. Lond. Math. Soc. (2) 89(3) (2014), 797-816. 
[31] Se-Goo Kim and Charles Livingston, 'Nonsplittability of the rational homology cobordism group of 3-manifolds', Pacific J. Math. 271(1) (2014), 183-211.

[32] Taehee Kim, 'Knots having the same Seifert form and primary decomposition of knot concordance', J. Knot Theory Ramifications 26(14) (2017), 1750103.

[33] Constance Leidy, 'Higher-order linking forms for knots', Comment. Math. Helv. 81(4) (2006), 755-781.

[34] Jerome P. Levine, 'Invariants of knot cobordism', Invent. Math. 8 (1969), 98-110; addendum, 8 (1969), 355.

[35] Jerome P. Levine, 'Knot cobordism groups in codimension two', Comment. Math. Helv. 44 (1969), $229-244$.

[36] Charles Livingston, 'Examples in concordance', (2001). URL: https://arxiv.org/abs/math/0101035v2.

[37] John W. Milnor, 'Infinite cyclic coverings', in Conference on the Topology of Manifolds (Michigan State University, East Lansing, MI, 1968), 115-133.

[38] András Némethi, 'On the Ozsváth-Szabó invariant of negative definite plumbed 3-manifolds', Geom. Topol. 9 (2005), 991-1042.

[39] Walter D. Neumann, 'A calculus for plumbing applied to the topology of complex surface singularities and degenerating complex curves', Trans. Amer. Math. Soc. 268(2) (1981), 299-344.

[40] Peter Ozsváth and Zoltán Szabó, 'Absolutely graded Floer homologies and intersection forms for four-manifolds with boundary', Adv. Math. 173(2) (2003), 179-261.

[41] Dale Rolfsen, Knots and Links, Mathematics Lecture Series, No. 7 (Publish or Perish Inc., Berkeley, CA, 1976).

[42] Desmond Sheiham, 'Invariants of boundary link cobordism', Mem. Amer. Math. Soc. 165(784) (2003), x+110.

[43] Desmond Sheiham, 'Invariants of boundary link cobordism. II. The Blanchfield-Duval form', in Non-commutative Localization in Algebra and Topology, (English summary), London Math. Soc. Lecture Note Ser., 330, (Cambridge Univ. Press, Cambridge, 2006), 143-219.

[44] Neal W. Stoltzfus, 'Unraveling the integral knot concordance group', Mem. Amer. Math. Soc. 12(192) (1977), iv+91.

[45] Ralph Strebel, 'Homological methods applied to the derived series of groups', Comment. Math. Helv. 49 (1974), $302-332$. 